\title{
THE TRENDS HIGH-CONTRAST IMAGING SURVEY. IV. THE OCCURRENCE RATE OF GIANT PLANETS AROUND M DWARFS
}

\author{
Benjamin T. Montet ${ }^{1}$, Justin R. Crepp ${ }^{2}$, John Asher Johnson ${ }^{3}$, Andrew W. Howard $^{4}$, And Geoffrey W. Marcy ${ }^{5}$ \\ ${ }^{1}$ Cahill Center for Astronomy and Astrophysics, California Institute of Technology, 1200 East California Boulevard, \\ MC 249-17, Pasadena, CA 91125, USA; btm@ astro.caltech.edu \\ ${ }^{2}$ Department of Physics, University of Notre Dame, 225 Nieuwland Science Hall, Notre Dame, IN 46656, USA \\ ${ }^{3}$ Harvard-Smithsonian Center for Astrophysics, 60 Garden Street, Cambridge, MA 02138, USA \\ ${ }^{4}$ Institute for Astronomy, University of Hawaii, 2680 Woodlawn Drive, Honolulu, HI 96822, USA \\ ${ }^{5}$ B-20 Hearst Field Annex, Astronomy Department, University of California, Berkeley, Berkeley, CA 94720, USA \\ Received 2013 July 22; accepted 2013 December 4; published 2014 January 2
}

\begin{abstract}
Doppler-based planet surveys have discovered numerous giant planets but are incomplete beyond several AU. At larger star-planet separations, direct planet detection through high-contrast imaging has proven successful, but this technique is sensitive only to young planets and characterization relies upon theoretical evolution models. Here we demonstrate that radial velocity measurements and high-contrast imaging can be combined to overcome these issues. The presence of widely separated companions can be deduced by identifying an acceleration (long-term trend) in the radial velocity of a star. By obtaining high spatial resolution follow-up imaging observations, we rule out scenarios in which such accelerations are caused by stellar binary companions with high statistical confidence. We report results from an analysis of Doppler measurements of a sample of 111 M-dwarf stars with a median of 29 radial velocity observations over a median time baseline of $11.8 \mathrm{yr}$. By targeting stars that exhibit a radial velocity acceleration ("trend") with adaptive optics imaging, we determine that $6.5 \% \pm 3.0 \%$ of M-dwarf stars host one or more massive companions with $1<m / M_{J}<13$ and $0<a<20 \mathrm{AU}$. These results are lower than analyses of the planet occurrence rate around higher-mass stars. We find the giant planet occurrence rate is described by a double power law in stellar mass $M$ and metallicity $F \equiv[\mathrm{Fe} / \mathrm{H}]$ such that $f(M, F)=0.039_{-0.028}^{+0.056} M^{0.8_{-0.9}^{+1.1}} 10^{(3.8 \pm 1.2) F}$. Our results are consistent with gravitational microlensing measurements of the planet occurrence rate; this study represents the first model-independent comparison with microlensing observations.
\end{abstract}

Key words: methods: observational - planets and satellites: detection - planets and satellites: fundamental parameters - techniques: high angular resolution - techniques: radial velocities

Online-only material: color figures

\section{INTRODUCTION}

Over the past 20 years, numerous planets have been detected by several different techniques, permitting the first estimates of the occurrence rate of planets orbiting stars in the solar neighborhood (e.g., Johnson et al. 2010b; Howard et al. 2010b; Gould et al. 2010; Vigan et al. 2012). As successful as these detection methods have been, each is sensitive only to a relatively narrow range of parameter space. For example, radial velocity (RV) studies are most sensitive to massive planets with orbital periods shorter than the time baseline of observations. Johnson et al. (2010b) find that $3.4_{-0.9}^{+2.2} \%$ of $\mathrm{M}$ dwarfs have a Saturn-mass or larger planet within 2.5 AU. Beyond a few AU, $\mathrm{RV}$ searches are incomplete as the time required for a planet to complete one orbit is longer than the typical observing baseline. Some studies have attempted to extrapolate beyond this boundary. For instance, Cumming et al. (2008) fit the observed $\mathrm{RV}$ planet population to a power law in planet mass and period and find that $18 \% \pm 1 \%$ of FGK stars host a Saturn-mass or larger planet within $20 \mathrm{AU}$. Recently, targeted RV surveys of M dwarfs have suggested the giant planet occurrence rate is significantly smaller for these diminutive stars. Bonfils et al. (2013) suggest that fewer than $1 \%$ of $\mathrm{M}$ dwarfs host a Saturn-mass or larger planet with an orbital period $1<P<10$ days, and $2_{-1}^{+3} \%$ host giant planets with orbital periods between 10 and 100 days.

Transit studies suffer from similar detection biases. Since a planet transits only once each orbit, several orbits must be observed to definitively confirm a planet, so characterization is limited to planets with periods shorter than a fraction of the observing baseline (Gaudi et al. 2005). Additionally, the probability of a planet transiting its host star decreases with increasing orbital period (Winn 2011), such that hundreds of thousands of stars must be monitored in order to study the planet population at $a \approx 1 \mathrm{AU}$ (Borucki \& Summers 1984). Nevertheless, the success of the Kepler mission (Borucki et al. 2010; Koch et al. 2010) has allowed for statistical analyses of transiting planets to be undertaken. For example, Morton \& Swift (2013) analyze M dwarfs included in the 2012 list of announced Kepler Objects of Interest (Batalha et al. 2013). By correcting for false positives (detections when no transiting planet exists), false negatives (nondetections when a transiting planet is present), and geometric effects (nondetections of nontransiting planets), they estimate an occurrence rate of 1.5 planets with periods of less than 90 days and radii larger than $0.5 R_{\oplus}$ per M-dwarf star. The occurrence rate found by these authors is slightly higher than previous analyses, which measure rates of approximately one planet per star (Youdin 2011; Mann et al. 2012; Swift et al. 2013; Dressing \& Charbonneau 2013).

Neither RV nor transit searches are yet conducive to the discovery and characterization of planets well beyond the "snow line," where water exists as ice. Instead, high contrast direct imaging techniques can be a powerful tool for detecting young planetary companions in this domain. The first direct imaging planet discoveries are securely in hand, including four 
companions to HR 8799 (Marois et al. 2008, 2010) and one each around $\beta$ Pictoris (Lagrange et al. 2009) and Gl 504 (Kuzuhara et al. 2013). ${ }^{6}$ Recent studies using these techniques have calculated an occurrence rate around A stars of $8.7_{-2.8}^{+10.1} \%$ at $1 \sigma$ confidence for planets larger than $3 M_{J}$ and separations between 5 and 320 AU (Vigan et al. 2012). Imaging studies have been most effective around high-mass stars (Crepp \& Johnson 2011; Carson et al. 2013). Nondetections around lower-mass stars have been used to place upper limits on the frequency of giant planets. For example, Nielsen \& Close (2010) rule out the presence of giant planets orbiting FGKM stars beyond 65 AU with $95 \%$ confidence. High-contrast imaging, while powerful, only provides a measure of the relative brightness of a companion. To estimate the companion's mass, the age of the star must be known and planetary thermal evolution models must be applied to estimate the temperature (and brightness) of the companion (Chabrier et al. 2000; Baraffe et al. 2003). Moreover, direct imaging is currently only sensitive to massive planets; the HR8799 planets and $\beta$ Pic b are believed to have masses $m>5 M_{J}$. RV and transit studies suggest such "superJupiters" are rare compared to Jovian-mass and smaller objects at smaller separations (Howard et al. 2010b, 2012).

The gravitational microlensing technique is also effective for finding giant planets in wide orbits and does not rely on planetary evolution models. Using this technique, planets can be detected by observing perturbations to the photometric gravitational microlensing signal when a planet and its host pass in front of a more distant star. Since $70 \%-75 \%$ of stars in the galaxy are M dwarfs, most lenses have mass $M<0.5 M_{\odot}$. Microlensing searches thus provide a measure of planet occurrence around low-mass stars. Microlensing studies are sensitive to planets near the Einstein ring, $R_{E} \sim 3.5 \mathrm{AU}\left(M / M_{\odot}\right)^{1 / 2}$, a much wider separation than RV and transit searches (Gould et al. 2010). Cassan et al. (2012) find microlensing searches are most sensitive to planets at a projected separation in the range $\left[s_{\max }^{-1} R_{E}, s_{\max } R_{E}\right]$, where $s_{\max } \sim\left(q / 10^{-4.3}\right)^{1 / 3}$ and $q$ is the mass ratio between a companion and the host star. These authors find a planet occurrence rate that can be parameterized by a double power-law function, in mass ratio $q$ and separation $s$, such that

$$
\frac{d^{2} N}{d \log q d \log s}=10^{-0.62 \pm 0.22}\left(\frac{q}{5 \times 10^{-4}}\right)^{-0.73 \pm 0.17} \mathrm{dex}^{-2}
$$

The normalization constant is equivalent to $0.24_{-0.10}^{+0.16}$. These results are calculated under the assumption that planets are distributed uniformly in $\log s$, as is the case for binary stars (Öpik 1924). Additionally, Sumi et al. (2010) find a power-law slope in mass such that $d N / d \log q \propto q^{-0.68 \pm 0.20}$ for Neptunesized planets, but do not attempt to quantify a normalization factor.

As microlensing studies focus on distant $\mathrm{M}$ dwarfs $(d>$ $1 \mathrm{kpc}$ ) in the direction of the galactic bulge (Gaudi et al. 2002), these stars can be difficult to characterize accurately due to crowding. Stellar masses and metallicities are often estimated without being measured spectroscopically. If these host stars have different masses than assumed, it would affect the results of planet occurrence rate studies by microlensing groups as these results do not account for correlations between stellar

\footnotetext{
6 Companions detected around Fomalhaut (Kalas et al. 2008; Currie et al. 2012), HD 95086 (Rameau et al. 2013), and LaCa15 (Kraus \& Ireland 2012) are also good candidates to be directly imaged planets, but their true nature is somewhat ambiguous.
}

and planet properties. Additionally, as microlensing searches are most sensitive near $r=R_{E}$, beyond approximately $10 \mathrm{AU}$, the lensing signal becomes very weak. At these separations, differentiating distant planets from unbound, "free-floating" planets becomes difficult (Sumi et al. 2011).

$\mathrm{RV}$ and microlensing studies probe different regions around a star, and extrapolations between the two domains suggest a possible discrepancy. Cassan et al. (2012) estimate a total giant planet occurrence rate significantly lower than the Cumming et al. (2008) RV result. Derived power-law distributions in mass may also be different for planets found by each method: Cumming et al. (2008) find a distribution such that $d N / d \log m \propto m^{-0.31 \pm 0.20}$ from RV-detected planets, while Cassan et al. (2012) find a distribution such that $d N / d \log q \propto q^{-0.73 \pm 0.17}$. Since microlensing studies target $\mathrm{M}$ dwarfs, which are confined to a narrow mass range, we can approximate $q=m / M$ as $m$. In this case, the microlensing result and RV result differ by $1.6 \sigma$. Since giant planet occurrence decreases with decreasing stellar mass and metallicity (Johnson et al. 2010a), the expected giant planet occurrence rate around M dwarfs would be smaller than that for FGK stars. Therefore, it is necessary to compare the microlensing planet population not to a population of FGK stars, but instead to a study of $\mathrm{RV}$-detected planets around M dwarfs.

Historically, RV observations have been used to detect and characterize planets once they complete a full orbit, limiting studies to planets with periods shorter than the observing time baseline. In this paradigm, potentially useful information is overlooked. Wide companions are not completely undetectable: instead they can be identified by the presence of long-term RV accelerations (linear "trends"), which can be used to infer the existence of a companion in a more distant orbit (Liu et al. 2002; Crepp et al. 2012a). However, a linear acceleration does not provide unique information about the mass and period of the companion - the same trend could be caused by a Jupitermass planet at $5 \mathrm{AU}$ or a $100 M_{J} \mathrm{M}$ dwarf at $25 \mathrm{AU}$. This degeneracy can be broken by adaptive optics (AO) imaging. Low-mass binary companions to nearby $\mathrm{M}$ dwarfs can be easily imaged by modern AO systems (Lloyd 2002; Siegler et al. 2003). Such detections form the basis for the TRENDS high-contrast imaging survey, which to date has detected four M dwarfs and one white dwarf companion to higher-mass stars (Crepp et al. 2012b, 2013a, 2013b).

In this work, we combine $\mathrm{RV}$ and $\mathrm{AO}$ observations of nearby cool stars to estimate the frequency of giant planets in wide orbits around $\mathrm{M}$ dwarfs. From a sample of $111 \mathrm{M}$ dwarfs observed with a median Doppler RV baseline of $11.8 \mathrm{yr}$, we identify four systems with long-term RV accelerations but no known companions and target these stars with AO imaging in an attempt to detect stellar-mass companions. We discuss these observations and our methodology in Section 2. Given an observed RV trend or lack thereof, we determine with high statistical confidence if a giant planet exists around each star. We analyze the effects of false positive and false negative detections of RV accelerations in our sample in Section 3. In Section 4, we estimate the occurrence rate of giant planets around $\mathrm{M}$ dwarfs and compare the measure to results from other techniques. We summarize and conclude in Section 5.

This study represents the first measurement of the planet population in the range 0-20 AU. While we rely on brown dwarf cooling models, our study does not make use of theoretical planetary evolution models, unlike other AO studies of planetary systems. 


\section{SAMPLE AND OBSERVATIONS}

\subsection{Target Selection}

Since 1997, the California Planet Search (CPS) collaboration has undertaken a comprehensive Doppler search for extrasolar planets at the Keck Observatory (e.g., Howard et al. 2010a). Using the Keck/High Resolution Echelle Spectrometer (HIRES; Vogt et al. 1994), the CPS program monitors over 2000 stars, most selected to be chromospherically quiet, single, and bright. Included in this sample is a collection of $\mathrm{M}$ dwarfs from the Gliese and Hipparcos catalogs brighter than $V=11.5$ and lacking known stellar companions within 2 arcsec (Rauscher \& Marcy 2006). This sample was later extended to $V=13.5$ and currently includes $131 \mathrm{M}$ dwarfs within $16 \mathrm{pc}$ of the Sun, where we define the $\mathrm{M}$ spectral class as targets with $B-V>1.44$.

To develop the sample used here, we first remove from this set 16 stars with a known, nearby stellar binary companion. We define "nearby" as a separation small enough that a test particle orbit with semimajor axis $\geqslant 30$ AU would be unstable, following the instability criterion of Holman \& Wiegert (1999). This criterion depends on the unknown eccentricity of the binary pair, as perturbative effects are maximized at periapsis. We take $e=0.5$ as a typical value and find the onset of instability occurs for binary stars with $a \sim 250$ AU. Planets can still form in these more compact binary systems (e.g., Gl667C; Anglada-Escudé et al. 2012a), but at such small separations protoplanetary disk formation and planet evolution would be affected significantly by the presence of stellar companions. This selection thus allows us to study a class of planets that likely followed similar evolutionary processes. Moreover, the detection of an acceleration around these stars is ambiguous, as it could be caused by the binary star, a planetary-mass companion, or both together.

After making the above selection, we are left with $111 \mathrm{RV}$ targets, all of which have at least $8 \mathrm{RV}$ observations and a time baseline longer than $2.9 \mathrm{yr}$. The median number of observations is 29 over a median time baseline of $11.8 \mathrm{yr}$. The stars have spectral types from M0 to M5.5 and masses in the range 0.64-0.10 $M_{\odot}$. Stellar masses are estimated using the empirical relation between mass and absolute $K$-band magnitude, $M_{K}$, described by Delfosse et al. (2000). We take 10\% as a typical uncertainty in the stellar mass, in line with previous estimates (Bean et al. 2006). $K$-band apparent magnitudes are measured using apparent magnitudes from the Two Micron All-Sky Survey (2MASS) point-source catalog (Skrutskie et al. 2006). The majority of our parallaxes are taken from an analysis of Hipparcos data (van Leeuwen 2007). Some of our stars were not observed by Hipparcos, while others have had their distances updated more recently. In these cases, we apply the distances listed in the SIMBAD astronomical database (Table 1). For example, for Gl 317, we use the parallax found by Anglada-Escudé et al. (2012b); their derived mass and metallicity are consistent with our estimated values. In all cases, stellar metallicities are estimated by measuring the offset between the star's position in the $\left\{V-K_{s}, M_{K_{S}}\right\}$ plane from a calibrated main sequence following the method of Neves et al. (2012). We take 0.17 dex as a typical uncertainty in the stellar metallicity, representative of the scatter between this photometric method and spectroscopic measures of stellar metallicity. Stellar parameters for these targets are listed in Table 1 and observational parameters are listed in Table 2. The distribution of RV observational parameters are shown in Figure 1. Spectral types are estimated by comparing the spectrum collected with HIRES to other spectra collected with this same instrument. RV observations for a representative sample of six "typical" stars are shown in Figure 2.

\subsection{Detecting Accelerations from Radial Velocities}

The detection of a long-term RV acceleration is facilitated by having many observations over a long time baseline to increase signal, but complicated by astrophysical "jitter" caused by rotational modulation of surface inhomogeneties. To determine the masses and semimajor axes to which we are sensitive to planetary companions, we inject a series of artificial companions into orbit around the stars in our sample. We define a logarithmically spaced grid of companion masses and semimajor axes spanning the range $0.75 M_{J}<m<100 M_{J}$ and $3 \mathrm{AU}<a<30 \mathrm{AU}$, such as the one shown in Figure 3. At each point, we inject 500 planets and randomly assign each of the remaining orbital elements. The longitude of ascending node $\Omega$, time of periapsis $t_{p}$, and argument of periapsis $\omega$ are drawn from a uniform distribution, while the inclination is drawn from a distribution $d n / d i=\sin i$ and the eccentricity from a distribution such that $d n / d e$ follows a beta distribution with $\alpha=1.12$ and $\beta=3.09$, which well replicates the distribution of observed eccentricities for RV planets with orbits longer than 382 days (Kipping 2013). We then numerically integrate these orbits forward in time over our true observing baseline.

At the epochs each star was observed by CPS, we calculate the expected RV signal caused by our injected planet. Each velocity is perturbed from the true expected Keplerian velocity by a normal variate with zero mean and standard deviation $\sigma$ representative of the total expected noise:

$$
\sigma=\sqrt{\sigma_{\gamma}^{2}+\sigma_{\mathrm{jitter}}^{2}}
$$

Here, $\sigma_{\gamma}$ is the photon noise, estimated for each individual observation by randomly selecting a single measurement of the measured Poisson photon noise from a true observation of the star. To account for the effects of jitter, we follow the method of Isaacson \& Fischer (2010), who develop an empirical relation between the level of stellar jitter, a star's $S_{\mathrm{HK}}$ value, and its $B-V$ color. $S_{\mathrm{HK}}$ is defined as the ratio of the flux in the Ca II line cores to flux in the surrounding continuum. We compare the $S_{\mathrm{HK}}$ value observed by CPS to that expected from the star's $B-V$ color, which provides an estimate of $\sigma_{\text {jitter. }}$ This value is added in quadrature to the photon noise to estimate a total observational uncertainty, $\sigma$. Typical observations carry a photon noise of $2-4 \mathrm{~m} \mathrm{~s}^{-1}$ and jitter values are typically $3-5 \mathrm{~m} \mathrm{~s}^{-1}$ for a total $\sigma$ value of 3-6 $\mathrm{m} \mathrm{s}^{-1}$ for the majority of stars. Median $\sigma$ values for each star are listed in Table 2.

Once all observations are accounted for, we search for evidence of our injected planetary companion, manifested as an acceleration in the RV data. Here, we define the existence of a trend using the Bayesian information criterion (BIC; Schwarz 1978; Bowler et al. 2010; Campo et al. 2011; Stevenson et al. 2012), which prefers simple, well-fitting models subject to

$$
\mathrm{BIC} \equiv-2 \ln \mathcal{L}+k \ln N,
$$

where $\mathcal{L}$ is the maximum likelihood for a model with $k$ free parameters and $N$ observations. The BIC thus favors models that fit the underlying data well, but penalizes increasingly complex models. For a more complex model to be preferred by the BIC, it must improve the fit by an amount greater than $k \ln N$ to overcome the penalty term. 
Table 1

M-dwarf Stars Analyzed in This Study

\begin{tabular}{|c|c|c|c|c|c|c|c|c|c|}
\hline Star & R.A. & Decl. & $\begin{array}{l}\text { Mass } \\
\left(M_{\odot}\right) \\
\end{array}$ & {$[\mathrm{Fe} / \mathrm{H}]$} & Spectral Type & $V$ & $V$ References & $\begin{array}{c}d \\
(\mathrm{pc}) \\
\end{array}$ & $d$ References \\
\hline Hip 428 & 00:05:10.9 & $+45: 47: 11.6$ & 0.53 & -0.07 & M1 & 9.93 & Gliese \& Jahreiß (1991) & 11.25 & van Leeuwen (2007) \\
\hline HD 225213 & $00: 05: 24.4$ & $-37: 21: 26.5$ & 0.39 & -0.42 & M1.5 & 8.57 & Koen et al. (2010) & 4.34 & van Leeuwen (2007) \\
\hline Hip 1734 & $00: 21: 56.0$ & $-31: 24: 21.8$ & 0.55 & 0.09 & M1.5 & 11.1 & Koen et al. (2010) & 17.98 & van Leeuwen (2007) \\
\hline G1 26 & $00: 38: 59.0$ & $+30: 36: 58.5$ & 0.43 & 0.02 & M2.5 & 11.2 & Høg et al. (2000) & 12.6 & van Altena et al. (1995) \\
\hline Hip 3143 & $00: 39: 58.8$ & $-44: 15: 11.6$ & 0.55 & -0.09 & M0.5 & 11.4 & Koen et al. (2010) & 23.99 & van Leeuwen (2007) \\
\hline Gl 48 & $01: 02: 32.2$ & $+71: 40: 47.3$ & 0.48 & 0.06 & M3 & 10.0 & Høg et al. (2000) & 8.24 & van Leeuwen (2007) \\
\hline Gl 49 & 01:02:38.9 & $+62: 20: 42.2$ & 0.58 & 0.06 & M1.5 & 9.56 & Høg et al. (2000) & 9.96 & van Leeuwen (2007) \\
\hline Hip 5643 & $01: 12: 30.6$ & -16.59 .56 .3 & 0.13 & -0.43 & M4.5 & 12.1 & Koen et al. (2010) & 3.69 & van Leeuwen (2007) \\
\hline Hip 8051 & $01: 43: 20.2$ & $+04: 19: 18.0$ & 0.41 & -0.16 & M2 & 10.9 & Koen et al. (2010) & 11.41 & van Leeuwen (2007) \\
\hline G1 83.1 & 02:00:13.0 & $+13: 03: 07.0$ & 0.15 & -0.31 & M4.5 & 12.3 & Landolt (1992) & 4.50 & van Leeuwen (2007) \\
\hline G244-047 & 02:01:35.3 & $+63: 46: 12.1$ & 0.48 & 0.07 & M3 & 11.0 & Høg et al. (2000) & 12.76 & van Altena et al. (1995) \\
\hline Gl 87 & 02:01:35.3 & $+63: 46: 12.1$ & 0.45 & -0.32 & M1.5 & 10.0 & Koen et al. (2010) & 10.41 & van Leeuwen (2007) \\
\hline Hip 11048 & $02: 22: 14.6$ & $+47: 52: 48.1$ & 0.62 & -0.08 & M0.5 & 9.41 & Gliese \& Jahreiß (1991) & 11.94 & van Leeuwen (2007) \\
\hline G1 105B & $02: 36: 15.3$ & $+06: 52: 19.1$ & 0.27 & -0.10 & M4 & 11.6 & Jenkins et al. (2009) & 7.73 & Jenkins et al. (2009) \\
\hline Gl 109 & $02: 44: 15.6$ & $+25: 31: 24.1$ & 0.35 & -0.18 & M3 & 10.6 & Koen et al. (2010) & 7.51 & van Leeuwen (2007) \\
\hline Hip 21556 & $04: 37: 42.9$ & $-11: 02: 19.9$ & 0.48 & -0.11 & M1.5 & 10.3 & Koen et al. (2010) & 11.10 & van Leeuwen (2007) \\
\hline Gl 179 & 04:52:05.7 & $+06: 28: 35.6$ & 0.36 & 0.13 & M3.5 & 12.0 & Koen et al. (2010) & 12.29 & van Leeuwen (2007) \\
\hline Hip 22762 & 04:59:50.0 & $-17: 46: 24.3$ & 0.42 & -0.20 & M2 & 10.9 & Koen et al. (2010) & 12.12 & van Leeuwen (2007) \\
\hline Hip 23512 & 05:03:20.1 & $-17: 22: 24.7$ & 0.27 & -0.25 & M3 & 11.7 & Koen et al. (2010) & 9.21 & van Leeuwen (2007) \\
\hline HD 33793 & 05:11:40.6 & $-45: 01: 06.3$ & 0.27 & -0.81 & M1 & 8.85 & Koen et al. (2010) & 3.91 & van Leeuwen (2007) \\
\hline Hip 24284 & $05: 12: 42.2$ & +19.39 .56 .4 & 0.45 & -0.16 & M2 & 10.7 & Koen et al. (2010) & 12.29 & van Leeuwen (2007) \\
\hline HD 36395 & $05: 31: 27.4$ & $-03: 40: 38.0$ & 0.60 & -0.05 & M1.5 & 7.92 & Koen et al. (2010) & 5.66 & van Leeuwen (2007) \\
\hline G097-054 & $05: 34: 52.1$ & $+13: 52: 47.2$ & 0.37 & 0.05 & M3.5 & 11.9 & Kharchenko (2001) & 12.39 & Gliese \& Jahreiß (1991) \\
\hline HD 233153 & 05:41:30.7 & $+53: 29: 23.3$ & 0.60 & 0.05 & M0.5 & 9.75 & Gliese \& Jahreiß (1991) & 12.44 & van Leeuwen (2007) \\
\hline Hip 26857 & $05: 42: 09.3$ & $+12.29: 21.6$ & 0.22 & -0.24 & M4 & 11.5 & Landolt (1992) & 5.83 & van Leeuwen (2007) \\
\hline G192-13 & 06:01:11.1 & $+59: 35: 50.8$ & 0.27 & -0.11 & M3.5 & 11.7 & van Altena et al. (1995) & 7.93 & Khrutskaya et al. (2010) \\
\hline Hip 29052 & 06:07:43.7 & $-25: 44: 41.5$ & 0.30 & -0.22 & M4 & 11.9 & Koen et al. (2010) & 11.35 & van Leeuwen (2007) \\
\hline G1 226 & $06: 10: 19.8$ & $+82.06: 24.3$ & 0.41 & -0.14 & M2 & 10.5 & Gliese \& Jahreiß (1991) & 9.37 & van Leeuwen (2007) \\
\hline G1 229B & $06: 10: 34.6$ & $-21: 51: 52.7$ & 0.58 & -0.07 & M1 & 8.13 & Koen et al. (2010) & 5.75 & van Leeuwen (2007) \\
\hline Gl 250B & $06: 52: 18.1$ & $-05: 11: 24.2$ & 0.45 & -0.12 & M2 & 10.1 & Gliese \& Jahreiß (1991) & 8.71 & van Leeuwen (2007) \\
\hline HD 265866 & $06: 54: 49.0$ & $+33: 16: 05.4$ & 0.35 & -0.03 & M3 & 10.11 & Høg et al. (2000) & 5.59 & van Leeuwen (2007) \\
\hline G1 273 & $07: 27: 24.5$ & $+05: 13: 32.8$ & 0.29 & -0.07 & M3.5 & 9.87 & Koen et al. (2010) & 3.80 & van Leeuwen (2007) \\
\hline Hip 36338 & $07: 28: 45.4$ & $-03: 17: 53.4$ & 0.40 & 0.03 & M3 & 11.4 & Koen et al. (2010) & 12.29 & van Leeuwen (2007) \\
\hline Hip 36834 & $07: 34: 27.4$ & $+62: 56: 29.4$ & 0.40 & -0.50 & M0.5 & 10.4 & Høg et al. (2000) & 11.47 & van Leeuwen (2007) \\
\hline Hip 37217 & 07:38:41.0 & $-21: 13: 28.5$ & 0.29 & -0.27 & M3 & 11.7 & Koen et al. (2010) & 10.60 & van Leeuwen (2007) \\
\hline Hip 37766 & $07: 44: 40.2$ & $+03: 33: 08.8$ & 0.31 & 0.27 & M4.5 & 11.2 & Koen et al. (2010) & 5.96 & van Leeuwen (2007) \\
\hline GJ 2066 & 08:16:08.0 & $+01: 18: 09.3$ & 0.46 & -0.10 & M2 & 10.1 & Koen et al. (2010) & 9.12 & van Leeuwen (2007) \\
\hline Gl 317 & 08:40:59.2 & $-23: 27: 23.3$ & 0.43 & 0.20 & M3.5 & 12.0 & van Altena et al. (1995) & 15.31 & Anglada-Escudé et al. (2012b) \\
\hline HD 75732B & $08: 52: 40.8$ & $+28: 18: 59.0$ & 0.27 & 0.15 & M4 & 13.1 & Gliese \& Jahreiß (1991) & 13.02 & Reid \& Cruz (2002) \\
\hline Hip 46655 & 09:30:44.6 & $+00: 19: 21.6$ & 0.29 & -0.17 & M3.5 & 11.7 & Koen et al. (2010) & 9.67 & van Leeuwen (2007) \\
\hline Hip 46769 & 09:31:56.3 & $+36.19: 12.8$ & 0.53 & -0.27 & M0 & 10.1 & Høg et al. (2000) & 13.91 & van Leeuwen (2007) \\
\hline Gl 357 & 09:36:01.6 & $-21: 39: 38.9$ & 0.33 & -0.31 & M2.5 & 10.9 & Koen et al. (2010) & 9.02 & van Leeuwen (2007) \\
\hline Hip 47513 & 09:41:10.4 & $+13: 12: 34.4$ & 0.48 & -0.12 & M1.5 & 10.4 & Koen et al. (2010) & 11.26 & van Leeuwen (2007) \\
\hline Hip 47650 & $09: 42: 51.7$ & $+70: 02: 21.9$ & 0.41 & 0.13 & M3 & 11.4 & Høg et al. (2000) & 11.35 & van Leeuwen (2007) \\
\hline Hip 48714 & 09:56:08.7 & $+62: 47: 18.5$ & 0.64 & -0.03 & M0 & 9.00 & Gliese \& Jahreiß (1991) & 10.56 & van Leeuwen (2007) \\
\hline Gl 382 & $10: 12: 17.7$ & $-03: 44: 44.4$ & 0.54 & 0.02 & M1.5 & 9.26 & Koen et al. (2010) & 7.87 & van Leeuwen (2007) \\
\hline Gl 388 & $10: 19: 36.3$ & $+19: 52: 10.1$ & 0.41 & 0.10 & M3.5 & 9.46 & Høg et al. (2000) & 4.69 & Jenkins et al. (2009) \\
\hline Hip 51007 & $10: 25: 10.8$ & $-10: 13: 43.3$ & 0.54 & -0.07 & M1 & 10.1 & Koen et al. (2010) & 12.35 & van Leeuwen (2007) \\
\hline Gl 393 & $10: 28: 55.6$ & $+00: 50: 27.6$ & 0.44 & -0.14 & M2 & 9.65 & Landolt (2009) & 7.07 & van Leeuwen (2007) \\
\hline Hip 53020 & $10: 50: 52.0$ & $+06: 48: 29.2$ & 0.26 & 0.00 & M4 & 11.7 & Landolt (1992) & 6.76 & van Leeuwen (2007) \\
\hline G1 406 & $10: 56: 28.9$ & $+07: 00: 52.8$ & 0.10 & 0.22 & M5.5 & 13.5 & Landolt (1992) & 2.39 & van Leeuwen (2007) \\
\hline Gl 408 & 11:00:04.3 & $+22: 49: 58.6$ & 0.38 & -0.15 & M2.5 & 10.0 & Koen et al. (2010) & 6.66 & van Leeuwen (2007) \\
\hline HD 95650 & $11: 02: 38.3$ & $+21: 58: 01.7$ & 0.59 & -0.10 & M0 & 9.57 & Koen et al. (2010) & 11.77 & van Leeuwen (2007) \\
\hline HD 95735 & $11: 03: 20.2$ & $+35.58: 11.6$ & 0.39 & -0.32 & M2 & 7.52 & Oja (1985) & 2.55 & van Leeuwen (2007) \\
\hline Hip 54532 & 11:09:31:3 & $-24: 35: 55.1$ & 0.46 & -0.08 & M2 & 10.4 & Koen et al. (2010) & 10.75 & van Leeuwen (2007) \\
\hline HD 97101B & 11:11:01.9 & $+30: 26: 44.4$ & 0.58 & 0.52 & M1.5 & 10.7 & Høg et al. (2000) & 11.87 & van Leeuwen (2007) \\
\hline Hip 55360 & 11:20:04.8 & $+65: 50: 47.3$ & 0.49 & -0.35 & M0 & 9.30 & Høg et al. (2000) & 8.92 & van Leeuwen (2007) \\
\hline G1 433 & $11: 35: 26.9$ & $-32: 32: 23.9$ & 0.47 & -0.15 & M1.5 & 9.81 & Koen et al. (2010) & 8.88 & van Leeuwen (2007) \\
\hline Hip 57050 & $11: 41: 44.6$ & $+42: 45: 07.1$ & 0.35 & 0.08 & M4 & 11.9 & Kharchenko (2001) & 11.10 & van Leeuwen (2007) \\
\hline Gl 436 & $11: 42: 11.2$ & $+26: 42: 22.6$ & 0.44 & -0.03 & M2.5 & 10.6 & Høg et al. (2000) & 10.14 & van Leeuwen (2007) \\
\hline Gl 445 & $11: 47: 41.4$ & $+78: 41: 28.2$ & 0.25 & -0.27 & M3.5 & 10.8 & Høg et al. (2000) & 5.35 & van Leeuwen (2007) \\
\hline Hip 57548 & $11: 47: 44.4$ & $+00: 48: 16.4$ & 0.17 & -0.23 & M4 & 11.1 & Landolt (1992) & 3.36 & van Leeuwen (2007) \\
\hline Gl 450 & 11:51:07.3 & $+35: 16: 19.3$ & 0.46 & -0.21 & M1 & 9.72 & Høg et al. (2000) & 8.59 & van Leeuwen (2007) \\
\hline Hip 59406 & 12:11:11.8 & $-19: 57: 38.1$ & 0.35 & -0.13 & M3 & 11.7 & Koen et al. (2010) & 12.59 & van Leeuwen (2007) \\
\hline Hip 59406b & $12: 11: 17.0$ & $-19: 58: 21.4$ & 0.25 & -0.25 & M4 & 12.6 & Gliese \& Jahreiß (1991) & 12.59 & van Leeuwen (2007) \\
\hline Hip 60559 & $12: 24: 52.5$ & $-18: 14: 32.2$ & 0.26 & -0.56 & M4 & 11.3 & Koen et al. (2010) & 8.85 & van Leeuwen (2007) \\
\hline Gl 486 & $12: 47: 56.6$ & $+09: 45: 05.0$ & 0.32 & 0.01 & M3.5 & 11.4 & Koen et al. (2010) & 8.37 & van Leeuwen (2007) \\
\hline Hip 63510 & 13:00:46.6 & $+12: 22: 36.6$ & 0.594 & 0.04 & M0.5 & 9.76 & Koen et al. (2010) & 11.4 & van Leeuwen (2007) \\
\hline
\end{tabular}


Table 1

(Continued)

\begin{tabular}{|c|c|c|c|c|c|c|c|c|c|}
\hline Star & R.A. & Decl. & $\begin{array}{l}\text { Mass } \\
\left(M_{\odot}\right)\end{array}$ & {$[\mathrm{Fe} / \mathrm{H}]$} & Spectral Type & $V$ & $V$ References & $\begin{array}{c}d \\
(\mathrm{pc})\end{array}$ & $d$ References \\
\hline Gl 514 & $13: 29: 59.8$ & $+10: 22: 37.8$ & 0.53 & -0.15 & M0.5 & 9.03 & Koen et al. (2010) & 7.66 & van Leeuwen (2007) \\
\hline HD 119850 & $13: 45: 43.8$ & $+14: 53: 29.5$ & 0.50 & -0.16 & M1.5 & 8.50 & van Belle \& von Braun (2009) & 5.39 & van Leeuwen (2007) \\
\hline Hip 67164 & $13: 45: 50: 7$ & $-17: 58: 05.6$ & 0.31 & -0.06 & M3.5 & 11.9 & Koen et al. (2010) & 10.24 & van Leeuwen (2007) \\
\hline HD 122303 & $14: 01: 03.2$ & $-02: 39: 17.5$ & 0.52 & -0.16 & M1 & 9.71 & Koen et al. (2010) & 10.03 & van Leeuwen (2007) \\
\hline Hip 70865 & $14: 29: 29.7$ & $+15: 31: 57.5$ & 0.52 & 0.00 & M2 & 10.7 & Koen et al. (2010) & 14.00 & van Leeuwen (2007) \\
\hline Hip 70975 & $14: 31: 01.2$ & $-12: 17: 45.9$ & 0.32 & -0.05 & M3.5 & 11.9 & Koen et al. (2010) & 10.82 & van Leeuwen (2007) \\
\hline Hip 71253 & $14: 34: 16.8$ & $-12: 31: 10.4$ & 0.28 & 0.11 & M4 & 11.3 & Koen et al. (2010) & 6.06 & van Leeuwen (2007) \\
\hline Hip 71898 & $14: 42: 21.6$ & $+66: 03: 20.9$ & 0.361 & -0.35 & M3 & 10.8 & Høg et al. (2000) & 9.87 & van Leeuwen (2007) \\
\hline G1 569A & $14: 54: 29.2$ & $+16: 06: 03.8$ & 0.48 & -0.03 & M2.5 & 10.2 & Koen et al. (2010) & 9.65 & van Leeuwen (2007) \\
\hline Gl 581 & $15: 19: 27.5$ & $-07: 43: 19.4$ & 0.30 & -0.18 & M3 & 10.6 & Høg et al. (2000) & 6.21 & van Leeuwen (2007) \\
\hline HD 147379B & $16: 16: 45.3$ & $+67: 15: 22.5$ & 0.47 & 0.09 & M3 & 10.7 & Gliese \& Jahreiß (1991) & 10.74 & van Leeuwen (2007) \\
\hline Gl 625 & $16: 25: 24.6$ & $+54: 18: 14.7$ & 0.32 & -0.39 & M1.5 & 10.2 & Høg et al. (2000) & 6.52 & van Leeuwen (2007) \\
\hline Gl 649 & $16: 58: 08.9$ & $+25: 44: 39.0$ & 0.54 & -0.10 & M1 & 9.66 & Høg et al. (2000) & 10.34 & van Leeuwen (2007) \\
\hline Hip 83762 & 17:07:07.5 & $+21: 33: 14.5$ & 0.38 & -0.10 & M3 & 11.7 & Koen et al. (2010) & 13.4 & van Leeuwen (2007) \\
\hline Hip 84099 & $17: 11: 34.7$ & $+38: 26: 33.9$ & 0.38 & -0.05 & M3.5 & 11.5 & Høg et al. (2000) & 12.00 & van Leeuwen (2007) \\
\hline Hip 84790 & $17: 19: 52.7$ & $+41: 42: 49.7$ & 0.37 & -0.21 & M2.5 & 11.4 & Gliese \& Jahreiß (1991) & 12.38 & van Leeuwen (2007) \\
\hline Gl 687 & $17: 36: 25.9$ & $+68: 20: 20.9$ & 0.40 & -0.06 & M3 & 9.15 & Høg et al. (2000) & 4.53 & van Leeuwen (2007) \\
\hline Gl 686 & $17: 37: 53.3$ & $+18: 35: 30.2$ & 0.44 & -0.31 & M1 & 9.58 & Koen et al. (2010) & 8.09 & van Leeuwen (2007) \\
\hline Gl 694 & $17: 43: 56.0$ & $+43: 22: 43.0$ & 0.44 & -0.02 & M2.5 & 10.5 & Høg et al. (2000) & 9.48 & van Leeuwen (2007) \\
\hline Gl 699 & $17: 57: 48.5$ & $+04: 41: 36.2$ & 0.16 & -0.61 & M4 & 9.51 & Koen et al. (2010) & 1.82 & van Leeuwen (2007) \\
\hline HD 165222 & $18: 05: 07.6$ & $-03: 01: 52.8$ & 0.48 & -0.22 & M1 & 9.36 & Koen et al. (2010) & 7.76 & van Leeuwen (2007) \\
\hline G205-028 & $18: 31: 58.4$ & $+40: 41: 10.4$ & 0.31 & -0.14 & M3.5 & 12.0 & Gliese \& Jahreiß (1991) & 11.9 & Browning et al. (2010) \\
\hline GJ 4063 & $18: 34: 36.6$ & $+40: 07: 26.4$ & 0.19 & -0.61 & M3.5 & 11.8 & Høg et al. (2000) & 7.25 & Reid \& Cruz (2002) \\
\hline Hip 91699 & $18: 41: 59.0$ & $+31: 49: 49.8$ & 0.37 & -0.13 & M3 & 11.3 & Kharchenko (2001) & 11.45 & van Leeuwen (2007) \\
\hline Hip 92403 & $18: 49: 49.4$ & $-23: 50: 10.4$ & 0.17 & -0.43 & M3.5 & 10.5 & Koen et al. (2010) & 2.97 & van Leeuwen (2007) \\
\hline G1 745A & 19:07:05.6 & $+20: 53: 17.0$ & 0.30 & -0.48 & M1.5 & 10.8 & Koen et al. (2010) & 8.51 & van Leeuwen (2007) \\
\hline G1 745B & 19:07:13.2 & $+20: 52: 37.2$ & 0.31 & -0.45 & M1.5 & 10.7 & Koen et al. (2010) & 8.75 & van Leeuwen (2007) \\
\hline G207-019 & 19:08:30.0 & $+32: 16: 52.0$ & 0.34 & -0.10 & M3 & 11.8 & Kharchenko (2001) & 12.39 & Browning et al. (2010) \\
\hline HD 180617 & $19: 16: 55.3$ & $+05: 10: 08.1$ & 0.48 & 0.02 & M2.5 & 9.12 & Koen et al. (2010) & 5.87 & van Leeuwen (2007) \\
\hline Gl 793 & $20: 30: 32.0$ & $+65: 26: 58.4$ & 0.38 & -0.03 & M2.5 & 10.7 & Høg et al. (2000) & 8.00 & van Leeuwen (2007) \\
\hline G1 806 & $20: 45: 04.1$ & $+44: 29.56 .7$ & 0.44 & -0.16 & M1.5 & 10.8 & Høg et al. (2000) & 12.32 & van Leeuwen (2007) \\
\hline Hip 103039 & $20: 52: 33.0$ & $-16: 58: 29.0$ & 0.23 & -0.10 & M4 & 11.4 & Koen et al. (2010) & 5.71 & van Leeuwen (2007) \\
\hline HD 199305 & $20: 53: 19.8$ & $+62: 09: 15.8$ & 0.58 & -0.02 & M0.5 & 8.60 & Høg et al. (2000) & 7.05 & van Leeuwen (2007) \\
\hline Hip 104432 & 21:09:17.4 & $-13: 18: 09.0$ & 0.36 & -0.51 & M1 & 10.9 & Landolt (2009) & 12.17 & van Leeuwen (2007) \\
\hline HD 209290 & $22: 02: 10.3$ & $+01: 24: 00.8$ & 0.60 & -0.10 & M0 & 9.15 & Koen et al. (2010) & 10.24 & van Leeuwen (2007) \\
\hline G1 849 & $22: 09: 40.3$ & $-04: 38: 26.6$ & 0.49 & 0.22 & M3.5 & 10.4 & Koen et al. (2010) & 9.10 & van Leeuwen (2007) \\
\hline Hip 109555 & $22: 11: 30.1$ & $+18: 25: 34.3$ & 0.55 & 0.13 & M2 & 10.2 & Koen et al. (2010) & 11.62 & van Leeuwen (2007) \\
\hline Gl 876 & $22: 53: 16.7$ & $-14: 15: 49.3$ & 0.34 & 0.13 & M4 & 10.2 & Landolt (2009) & 4.69 & van Leeuwen (2007) \\
\hline HD 216899 & $22: 56: 34.8$ & $+16: 33: 12.4$ & 0.58 & 0.03 & M1.5 & 8.64 & Koen et al. (2010) & 6.84 & van Leeuwen (2007) \\
\hline HD 217987 & $23: 05: 52.0$ & $-35: 51: 11.0$ & 0.47 & -0.33 & M0.5 & 7.34 & Gliese \& Jahreiß (1991) & 3.28 & van Leeuwen (2007) \\
\hline Hip 114411 & $23: 10: 15.7$ & $-25: 55: 52.7$ & 0.46 & -0.13 & $\mathrm{M} 2$ & 11.3 & Koen et al. (2010) & 16.08 & van Leeuwen (2007) \\
\hline Hip 115332 & $23: 21: 37.4$ & $+17: 17: 25.4$ & 0.40 & 0.27 & M4 & 11.7 & Koen et al. (2010) & 10.99 & van Leeuwen (2007) \\
\hline Hip 115562 & $23: 24: 30.5$ & $+57: 51: 15.5$ & 0.59 & 0.08 & M1 & 10.0 & Gliese \& Jahreiß (1991) & 12.96 & van Leeuwen (2007) \\
\hline Gl 905 & $23: 41: 55.0$ & $+44: 10: 40.8$ & 0.14 & 0.05 & M5 & 12.3 & Jenkins et al. (2009) & 3.16 & Gatewood (2008) \\
\hline Gl 908 & $23: 49: 12.5$ & $+02: 24: 04.4$ & 0.42 & -0.39 & M1 & 8.99 & Landolt (2009) & 5.98 & van Leeuwen (2007) \\
\hline
\end{tabular}

Note. Metallicity uncertainties are taken to be 0.17 dex, while mass uncertainties are taken as $10 \%$, following the method of Delfosse et al. (2000).

Kass \& Raftery (1995) claim a difference between BIC values provides a bounded approximation of twice the logarithm of the Bayes factor. A change in BIC value of 10 or more (corresponding to a Bayes factor of approximately 0.01) suggests strong evidence for an association between two parameters. If the BIC value decreases by more than 10 when considering a model with a linear acceleration over a model with only an offset, a planet is considered to be detected. Otherwise, the system is considered a nondetection. We find that the $\triangle \mathrm{BIC}$ value chosen here is consistent with by-eye inspection of our data in a visual search for RV accelerations. In both cases, we allow for a linear offset in the RV data in 2004 August, corresponding to an upgrade of the HIRES CCD detector (Wright et al. 2011). Effectively, we treat the data from before and after the upgrade as coming from two distinct instruments, which serves to slightly decrease our sensitivity to small RV accelerations.
By repeating this process for many simulated planets over our mass-semimajor axis grid, we can map out the relative probability of detecting a linear trend caused by a planet as a function of companion mass and semimajor axis. As an example, Figure 3 shows RVs for HIP 70975 and the likelihood of detecting a planet at a given mass and period given these observations. Figure 4 shows the mean likelihood of detecting a planet around a given star across our sample. Throughout this work, we report the occurrence rate of planets with masses in the range $1 M_{J}<m<13 M_{J}$. We can detect accelerations caused by planets smaller than $1 M_{J}$ in certain instances, but would miss the majority of these planets. As Figure 4 shows, we can only detect a $0.75 M_{J}$ planet at $6 \mathrm{AU} 50 \%$ of the time; planets at smaller separations would exhibit significant curvature over a $12 \mathrm{yr}$ time baseline and could be detected through an RV survey alone. We are more efficient at detecting planets larger than 
Table 2

RV Observations

\begin{tabular}{|c|c|c|c|c|c|c|c|}
\hline$\overline{\text { Star }}$ & $N_{\text {obs }}$ & $\begin{array}{c}\text { Baseline } \\
(\mathrm{yr})\end{array}$ & $\begin{array}{l}\text { Med. } \sigma_{\gamma} \\
\left(\mathrm{m} \mathrm{s}^{-1}\right)\end{array}$ & $\begin{array}{c}\text { Jitter } \\
\left(\mathrm{m} \mathrm{s}^{-1}\right)\end{array}$ & $\begin{array}{c}\mathrm{rms} \\
\left(\mathrm{m} \mathrm{s}^{-1}\right)\end{array}$ & RV Planets & Binary Companion \\
\hline Hip 428 & 41 & 12.2 & 1.6 & 4.2 & 4.8 & 0 & K6 (Bidelman 1954) \\
\hline HD 225213 & 67 & 9.9 & 1.1 & 3.2 & 3.1 & 0 & $\ldots$ \\
\hline Hip 1734 & 8 & 8.1 & 2.6 & 4.7 & 7.6 & 0 & $\cdots$ \\
\hline Gl 26 & 40 & 11.6 & 2.8 & 2.9 & 7.7 & 0 & $\ldots$ \\
\hline Hip 3143 & 8 & 9.8 & 5.6 & 2.6 & 11.6 & 0 & $\cdots$ \\
\hline Gl 48 & 41 & 15.2 & 1.3 & 2.5 & 3.5 & 0 & $\cdots$ \\
\hline Gl 49 & 22 & 14.2 & 1.4 & 7.9 & 5.0 & 0 & $\ldots$ \\
\hline Hip 5643 & 15 & 7.1 & 3.4 & 13.2 & 7.8 & 0 & $\cdots$ \\
\hline Hip 8051 & 33 & 12.7 & 1.5 & 3.0 & 5.0 & 0 & $\cdots$ \\
\hline Gl 83.1 & 21 & 8.2 & 3.3 & 12.5 & 20.2 & 0 & $\ldots$ \\
\hline G244-047 & 10 & 7.5 & 2.8 & 3.6 & 4.0 & 0 & $\ldots$ \\
\hline G1 87 & 62 & 13.0 & 1.3 & 2.5 & 7.4 & 0 & $\cdots$ \\
\hline Hip 11048 & 44 & 12.6 & 1.1 & 4.8 & 5.4 & 0 & $\ldots$ \\
\hline Gl 105B & 12 & 9.1 & 2.7 & 3.7 & 13.0 & 0 & K3 (Gray et al. 2006) \\
\hline Gl 109 & 32 & 13.1 & 1.4 & 2.8 & 4.4 & 0 & $\ldots$ \\
\hline Hip 21556 & 31 & 12.7 & 1.3 & 2.5 & 4.3 & 0 & $\ldots$ \\
\hline Gl 179 & 42 & 12.2 & 2.5 & 4.4 & 19.7 & 1 & $\cdots$ \\
\hline Hip 22762 & 39 & 12.6 & 1.6 & 2.7 & 4.6 & 0 & $\cdots$ \\
\hline Hip 23512 & 11 & 6.7 & 4.1 & 5.0 & 6.7 & 0 & $\ldots$ \\
\hline HD 33793 & 36 & 13.8 & 1.4 & 2.9 & 3.2 & 0 & $\cdots$ \\
\hline Hip 24284 & 30 & 9.1 & 1.4 & 2.3 & 5.4 & 0 & $\cdots$ \\
\hline HD 36395 & 33 & 15.8 & 1.7 & 5.7 & 7.8 & 0 & $\ldots$ \\
\hline G097-054 & 11 & 6.6 & 3.6 & 3.4 & 8.7 & 0 & $\cdots$ \\
\hline HD 233153 & 11 & 6.7 & 2.3 & 5.8 & 6.6 & 0 & K1 (Montes et al. 2001) \\
\hline Hip 26857 & 10 & 6.7 & 4.7 & 4.6 & 11.8 & 0 & $\cdots$ \\
\hline G192-13 & 16 & 7.8 & 4.3 & 4.1 & 11.4 & 0 & $\cdots$ \\
\hline Hip 29052 & 16 & 7.7 & 4.6 & 3.5 & 10.5 & 0 & $\cdots$ \\
\hline G1 226 & 35 & 14.7 & 1.6 & 2.3 & 8.7 & 0 & $\ldots$ \\
\hline Gl 229B & 33 & 15.9 & 1.2 & 4.5 & 5.1 & 0 & T7 (Faherty et al. 2009) \\
\hline Gl 250B & 29 & 8.0 & 1.3 & 3.7 & 3.4 & 0 & K3 (Gliese \& Jahreiß 1991) \\
\hline HD 265866 & 61 & 14.8 & 1.3 & 2.6 & 4.6 & 0 & $\cdots$ \\
\hline Gl 273 & 41 & 14.8 & 2.1 & 2.3 & 5.0 & 0 & $\cdots$ \\
\hline Hip 36338 & 10 & 10.7 & 2.9 & 2.3 & 5.8 & 0 & $\cdots$ \\
\hline Hip 36834 & 22 & 6.4 & 2.7 & 5.8 & 14.6 & 0 & $\ldots$ \\
\hline Hip 37217 & 11 & 11.8 & 3.4 & 25.7 & 5.3 & 0 & $\cdots$ \\
\hline Hip 37766 & 22 & 11.1 & 3.1 & 87.9 & 95.2 & 0 & $\cdots$ \\
\hline GJ 2066 & 37 & 14.8 & 1.5 & 2.5 & 5.3 & 0 & $\cdots$ \\
\hline Gl 317 & 45 & 12.1 & 2.2 & 4.5 & 56.9 & 1 & $\cdots$ \\
\hline HD 75732B & 21 & 9.1 & 5.2 & 4.9 & 17.1 & 0 & G8 (Montes et al. 2001) \\
\hline Hip 46655 & 11 & 6.0 & 3.9 & 2.9 & 18.6 & 0 & $\cdots$ \\
\hline Hip 46769 & 23 & 8.0 & 1.4 & 3.5 & 6.3 & 0 & $\cdots$ \\
\hline Gl 357 & 36 & 14.2 & 1.8 & 2.1 & 6.1 & 0 & $\cdots$ \\
\hline Hip 47513 & 29 & 12.1 & 1.4 & 3.8 & 6.1 & 0 & $\cdots$ \\
\hline Hip 47650 & 10 & 6.2 & 3.2 & 16.2 & 11.0 & 0 & $\cdots$ \\
\hline Hip 48714 & 16 & 11.2 & 1.4 & 6.3 & 9.6 & 0 & $\ldots$ \\
\hline Gl 382 & 29 & 12.9 & 1.5 & 5.3 & 6.4 & 0 & $\ldots$ \\
\hline Gl 388 & 39 & 5.7 & 1.8 & 24.0 & 17.9 & 0 & $\cdots$ \\
\hline Hip 51007 & 19 & 11.1 & 2.2 & 4.2 & 6.1 & 0 & $\ldots$ \\
\hline Gl 393 & 42 & 14.4 & 1.2 & 3.3 & 3.9 & 0 & $\cdots$ \\
\hline Hip 53020 & 12 & 6.3 & 3.4 & 6.5 & 13.0 & 0 & $\cdots$ \\
\hline Gl 406 & 21 & 13.0 & 6.8 & 20.1 & 15.0 & 0 & $\cdots$ \\
\hline Gl 408 & 39 & 14.8 & 1.4 & 3.1 & 4.2 & 0 & $\cdots$ \\
\hline HD 95650 & 30 & 11.1 & 1.8 & 10.8 & 14.8 & 0 & $\cdots$ \\
\hline HD 95735 & 211 & 15.2 & 1.0 & 2.7 & 3.9 & 0 & $\cdots$ \\
\hline Hip 54532 & 26 & 12.2 & 2.6 & 2.9 & 12.9 & 0 & $\cdots$ \\
\hline HD 97101B & 25 & 10.5 & 1.4 & 4.7 & 4.7 & 0 & K8 (Gliese \& Jahreiß 1991) \\
\hline Hip 55360 & 30 & 11.9 & 2.4 & 2.2 & 8.2 & 0 & $\cdots$ \\
\hline Gl 433 & 27 & 13.1 & 2.4 & 2.4 & 6.8 & 0 & $\cdots$ \\
\hline Hip 57050 & 40 & 11.8 & 3.1 & 3.4 & 25.9 & 1 & $\cdots$ \\
\hline Gl 436 & 257 & 12.0 & 1.7 & 2.2 & 12.0 & 1 & $\ldots$ \\
\hline Gl 445 & 48 & 13.3 & 1.7 & 2.4 & 7.0 & 0 & $\cdots$ \\
\hline Hip 57548 & 17 & 12.8 & 2.8 & 9.2 & 5.9 & 0 & $\ldots$ \\
\hline Gl 450 & 31 & 14.1 & 2.0 & 4.7 & 7.0 & 0 & $\cdots$ \\
\hline Hip 59406 & 11 & 7.0 & 4.4 & 2.2 & 11.4 & 0 & M4 (Table 1) \\
\hline Hip 59406b & 12 & 6.2 & 6.1 & 3.2 & 13.2 & 0 & M3 (Table 1) \\
\hline
\end{tabular}


Table 2

(Continued)

\begin{tabular}{|c|c|c|c|c|c|c|c|}
\hline Star & $N_{\mathrm{obs}}$ & $\begin{array}{c}\text { Baseline } \\
(\mathrm{yr})\end{array}$ & $\begin{array}{r}\text { Med. } \sigma_{\gamma} \\
\left(\mathrm{m} \mathrm{s}^{-1}\right)\end{array}$ & $\begin{array}{c}\text { Jitter } \\
\left(\mathrm{m} \mathrm{s}^{-1}\right)\end{array}$ & $\begin{array}{c}\mathrm{rms} \\
\left(\mathrm{m} \mathrm{s}^{-1}\right)\end{array}$ & RV Planets & Binary Companion \\
\hline Hip 60559 & 14 & 6.3 & 3.4 & 3.1 & 8.9 & 0 & $\ldots$ \\
\hline Gl 486 & 20 & 8.2 & 3.0 & 2.5 & 11.3 & 0 & $\ldots$ \\
\hline Hip 63510 & 41 & 11.3 & 3.4 & 6.0 & 1011.0 & 0 & M7 (Beuzit et al. 2004) \\
\hline Gl 514 & 50 & 13.9 & 1.4 & 3.5 & 6.0 & 0 & $\cdots$ \\
\hline HD 119850 & 42 & 13.9 & 1.3 & 2.2 & 3.2 & 0 & $\cdots$ \\
\hline Hip 67164 & 14 & 6.2 & 4.0 & 2.2 & 8.3 & 0 & $\ldots$ \\
\hline HD 122303 & 37 & 11.8 & 1.3 & 3.4 & 6.9 & 0 & $\cdots$ \\
\hline Hip 70865 & 21 & 8.5 & 1.8 & 2.7 & 7.5 & 0 & $\cdots$ \\
\hline Hip 70975 & 15 & 11.3 & 2.9 & 2.8 & 8.5 & 0 & $\ldots$ \\
\hline Hip 71253 & 21 & 7.9 & 2.7 & 4.2 & 8.1 & 0 & $\ldots$ \\
\hline Hip 71898 & 30 & 14.1 & 2.4 & 2.9 & 41.0 & 0 & L0 (Faherty et al. 2009) \\
\hline Gl 569A & 13 & 5.1 & 2.5 & 14.7 & 6.6 & 0 & M8.5+M9 (Mason et al. 2001) \\
\hline Gl 581 & 197 & 12.5 & 1.3 & 2.8 & 9.9 & 4 & $\cdots$ \\
\hline HD 147379B & 14 & 5.9 & 2.2 & 4.1 & 4.8 & 0 & M1 (Herbig 2007) \\
\hline Gl 625 & 48 & 14.0 & 1.7 & 2.7 & 3.6 & 0 & $\ldots$ \\
\hline Gl 649 & 50 & 12.6 & 1.4 & 5.6 & 9.4 & 1 & $\cdots$ \\
\hline Hip 83762 & 8 & 2.9 & 1.3 & 2.8 & 7.1 & 0 & $\cdots$ \\
\hline Hip 84099 & 16 & 6.2 & 2.8 & 2.6 & 6.6 & 0 & $\cdots$ \\
\hline Hip 84790 & 17 & 4.9 & 3.0 & 2.2 & 5.6 & 0 & $\cdots$ \\
\hline Gl 687 & 100 & 13.8 & 1.2 & 2.3 & 5.9 & 0 & M3.5 (Jenkins et al. 2009) \\
\hline Gl 686 & 60 & 14.4 & 1.1 & 2.4 & 3.4 & 0 & $\ldots$ \\
\hline Gl 694 & 38 & 14.4 & 2.2 & 3.1 & 4.6 & 0 & $\cdots$ \\
\hline G1 699 & 230 & 15.3 & 1.3 & 7.0 & 4.1 & 0 & $\ldots$ \\
\hline HD 165222 & 142 & 14.4 & 1.2 & 3.1 & 3.4 & 0 & $\ldots$ \\
\hline G205-028 & 12 & 6.2 & 3.8 & 27.6 & 8.1 & 0 & $\cdots$ \\
\hline GJ 4063 & 14 & 6.9 & 2.7 & 2.5 & 6.1 & 0 & $\ldots$ \\
\hline Hip 91699 & 17 & 12.0 & 2.9 & 3.4 & 11.6 & 0 & $\cdots$ \\
\hline Hip 92403 & 27 & 8.1 & 2.8 & 7.7 & 18.8 & 0 & $\cdots$ \\
\hline Gl 745A & 26 & 13.3 & 1.5 & 2.9 & 3.9 & 0 & M1.5 (Table 1) \\
\hline Gl 745B & 21 & 10.4 & 2.5 & 2.9 & 5.5 & 0 & M1.5 (Table 1) \\
\hline G207-019 & 12 & 6.2 & 3.3 & 9.7 & 7.9 & 0 & $\cdots$ \\
\hline HD 180617 & 143 & 9.8 & 1.3 & 3.3 & 4.7 & 0 & M8 (Jenkins et al. 2009) \\
\hline Gl 793 & 30 & 14.2 & 1.6 & 4.9 & 5.0 & 0 & $\cdots$ \\
\hline G1 806 & 63 & 15.3 & 1.6 & 3.1 & 6.5 & 0 & $\cdots$ \\
\hline Hip 103039 & 19 & 8.2 & 3.4 & 5.5 & 6.7 & 0 & $\ldots$ \\
\hline HD 199305 & 45 & 15.3 & 1.1 & 4.5 & 3.3 & 0 & $\cdots$ \\
\hline Hip 104432 & 34 & 12.3 & 1.7 & 3.1 & 5.0 & 0 & $\cdots$ \\
\hline HD 209290 & 56 & 11.0 & 1.0 & 4.6 & 3.7 & 0 & $\cdots$ \\
\hline Gl 849 & 84 & 14.4 & 1.6 & 3.1 & 21.5 & 1 & $\cdots$ \\
\hline Hip 109555 & 16 & 11.1 & 2.5 & 12.5 & 8.4 & 0 & $\cdots$ \\
\hline Gl 876 & 207 & 14.4 & 2.1 & 4.0 & 150.4 & 4 & $\cdots$ \\
\hline HD 216899 & 50 & 15.1 & 1.1 & 4.2 & 4.6 & 0 & M2 (Zakhozhaj 2002) \\
\hline HD 217987 & 69 & 14.3 & 1.2 & 3.3 & 4.9 & 0 & $\ldots$ \\
\hline Hip 114411 & 11 & 8.9 & 2.7 & 3.3 & 7.2 & 0 & $\ldots$ \\
\hline Hip 115332 & 14 & 6.7 & 3.4 & 3.2 & 9.2 & 0 & $\cdots$ \\
\hline Hip 115562 & 10 & 8.8 & 1.6 & 6.2 & 9.0 & 0 & $\cdots$ \\
\hline Gl 905 & 17 & 8.0 & 3.8 & 8.6 & 8.8 & 0 & $\ldots$ \\
\hline Gl 908 & 89 & 16.0 & 1.2 & 2.6 & 2.9 & 0 & $\cdots$ \\
\hline
\end{tabular}

$1 M_{J}$, although we would still not expect to detect all planets in this range. We account for false negative "missed" planets in our analysis, as described in Section 3.1.

Eight of the stars in our sample host known planets with closed orbits. All of the planets have $m \sin i<2.5 M_{J}$ and are listed in Table 3 . To identify RV accelerations caused by outer planets, we include the signal from these planets by comparing a model which contains the known planet and an acceleration to a model which contains only the known planet. Two known planets in our sample, Gl 876b and Gl 317b, are larger than $1 M_{J}$, so in addition to searching these systems for long-term $\mathrm{RV}$ accelerations, we also include these known planets in our giant planet occurrence calculations.
One additional planet, Gl 649b, has a best-fitting mass $m \sin i=0.90 \pm 0.05 M_{J}$; if the inclination is smaller than $64^{\circ}$ this planet has mass $m>1 M_{J}$. We follow the method of Ho \& Turner (2011) to determine the probability of this event. That is, we define the probability that the true mass $m$ is greater than some value $X$ given an observed mass $m_{O}=m \sin i$ such that

$$
P\left(m>X \mid m_{O}\right)=1-\frac{\int_{m_{O}}^{X} \frac{\left(m_{O} / m^{2}\right)}{\sqrt{1-\left(m_{O} / m\right)^{2}}} P(m) d m}{\int_{m_{O}}^{m_{\max }} \frac{\left(m_{O} / m^{2}\right)}{\sqrt{1-\left(m_{O} / m\right)^{2}}} P(m) d m} .
$$

Here, $P(m)$ is the true planet mass distribution function. $m_{\max }$ is the physical upper mass limit for a planet. Since the true 

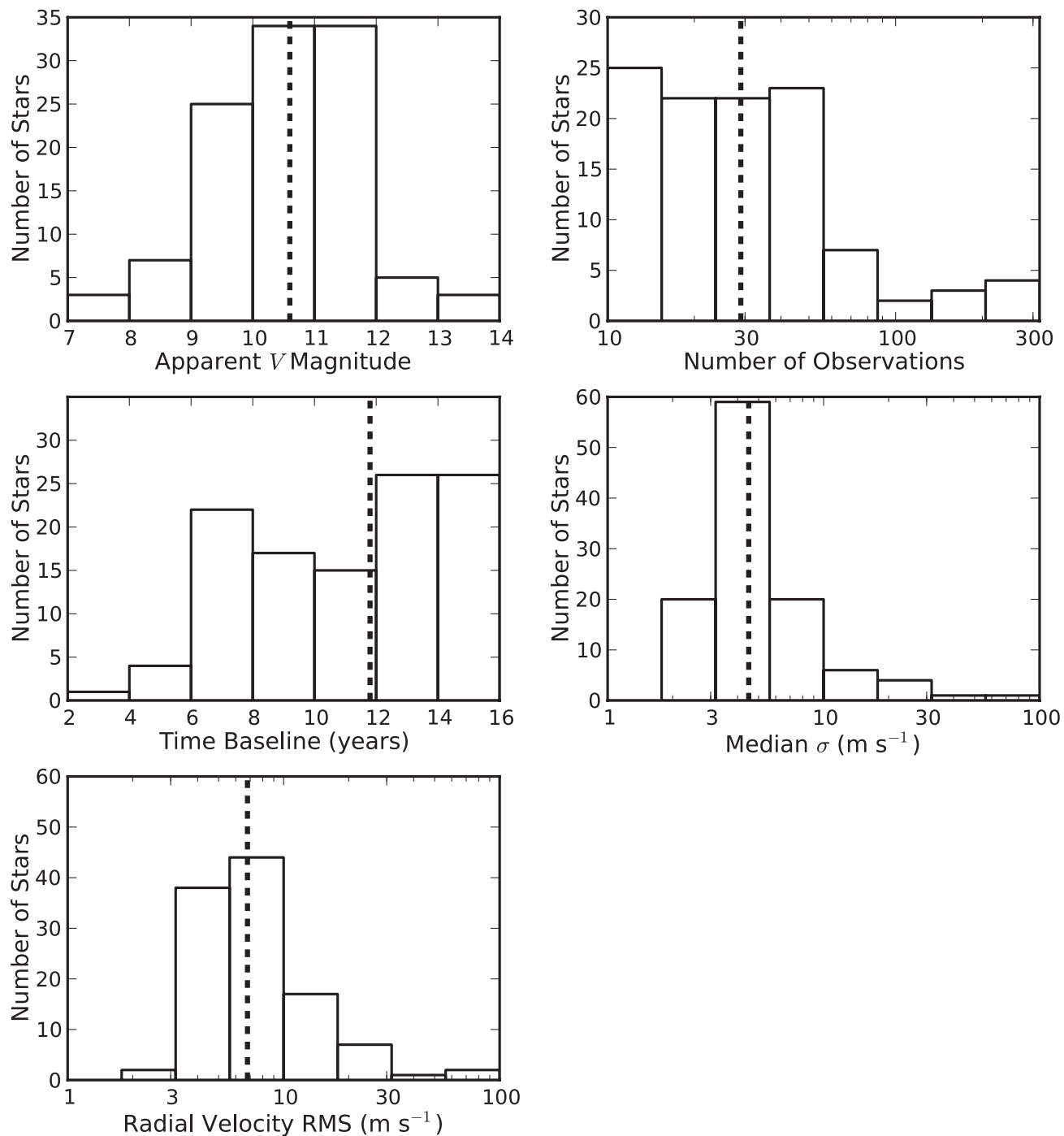

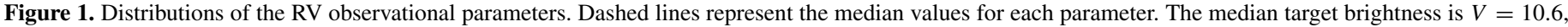

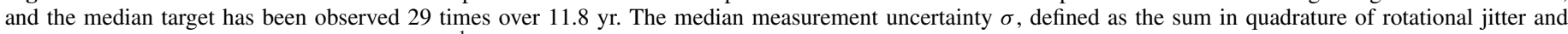
statistical uncertainty (Equation (2)) is $4.5 \mathrm{~m} \mathrm{~s}^{-1}$. Specific parameters for each individual system are shown in Table 1.

distribution function is strongly biased toward small planets, the number selected here does not significantly affect our results. By simply assuming the star is aligned randomly along our line of sight so that the inclination distribution is flat in $\cos i$, the result of a flat planet mass distribution function, we expect a observed mass $m \sin i=0.90 M_{J}$ to be produced by a Jupiter-mass or larger planet $56 \%$ of the time; all reasonable assumptions of an underlying mass distribution affect this value by less than $10 \%$. We repeat this procedure for all confirmed planets in our sample with masses $m \sin i<1 M_{J}$ to quantify the likelihood that other known planets are $m>1 M_{J}$ planets with low inclinations. We find, in addition to G1 849b, HIP 22627b ( $\left.m \sin i=0.64 M_{J}\right)$ has approximately a $25 \%$ probability of having a mass $m>1 M_{J}$. This probability is vanishingly small for all other known planets.

Of our sample of 111 stars, two have confirmed planets larger than $1 M_{J}$, six systems have confirmed RV planets with masses $m \sin i<1 M_{J}$ only, two exhibit RV acceleration caused by known brown dwarfs, and four show unexplained long-term $\mathrm{RV}$ accelerations, such that $\Delta \mathrm{BIC}>10$ when we include an acceleration term in our fit to the RV data. In the case of Gl 849b, the long-term acceleration exhibits significant curvature, so we are able to place constraints on this object's mass and orbital semimajor axis (see Appendix A). In all other cases, the magnitude of the observed acceleration is different from zero by $3 \sigma$. Additionally, the magnitude of the acceleration is such that over the observing baseline, the expected $\Delta \mathrm{RV}$ induced by the putative outer planet is larger than the uncertainties of each individual data point. The distribution of these systems in the stellar mass-metallicity plane is shown in Figure 5.

For the four targets with an observable RV drift, we create a grid of logarithmically spaced companion masses and semimajor axes over the range $0.75<m / M_{J}<100$ and $3 \mathrm{AU}<a<30 \mathrm{AU}$. For a given grid point, we determine the best-fitting Keplerian orbit for a given eccentricity and inclination. We assume the inclination and eccentricity distributions are the same as assumed previously. The eccentricity distribution is well characterized for solar-type stars, but may not hold for planets around lower-mass stars. We find the exact choice of eccentricity distribution does not significantly affect our results.

We determine the likelihood of the best-fitting orbit for each mass, period, eccentricity, and inclination. We then convert these likelihoods into relative probabilities, assuming our errors are uncorrelated so that $P \propto-\exp \left(\chi^{2} / 2\right)$. We then marginalize over eccentricity and inclination and normalize our probabilities 


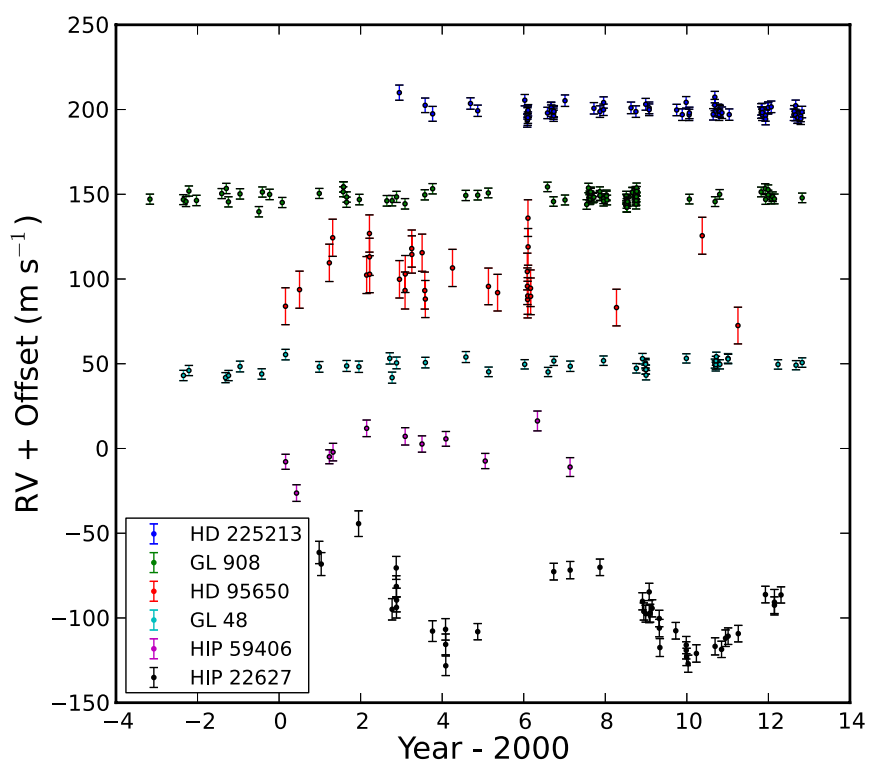

Figure 2. RV measurements for a representative sample of six example stars. The stars are arranged such that the brightest star is at the top of the plot. The individual stars vary considerably with respect to observing baselines, measurement uncertainty, and number of observations. Of these stars, HIP 59406 has a wide binary companion, while HIP 22627 has both a known inner planet and long-term RV acceleration.

(A color version of this figure is available in the online journal.)

so that $\sum_{M, a} P=1$. In these cases, we assume the inclination is random on the sky, so that the inclination follows the distribution $f(i)=\sin i$. Assuming a different planet mass distribution function affects this result by less than $10 \%$. The result is a contour in the mass-semimajor axis plane for the likelihood that a given object could cause the observed stellar RV variation (Wright et al. 2007). An example is shown in Figure 6. Implicit in this analysis is the assumption the RV variation is dominated by the motion of a single, massive companion rather than the constructive interference of the RV signal of two or more smaller objects. We discuss false positive probabilities in Section 3.2 and conclude the assumption that one signal dominates the observed $\mathrm{RVs}$ is reasonable.

The magnitude of an acceleration depends on both the semimajor axis and mass of the companion. For a planet in

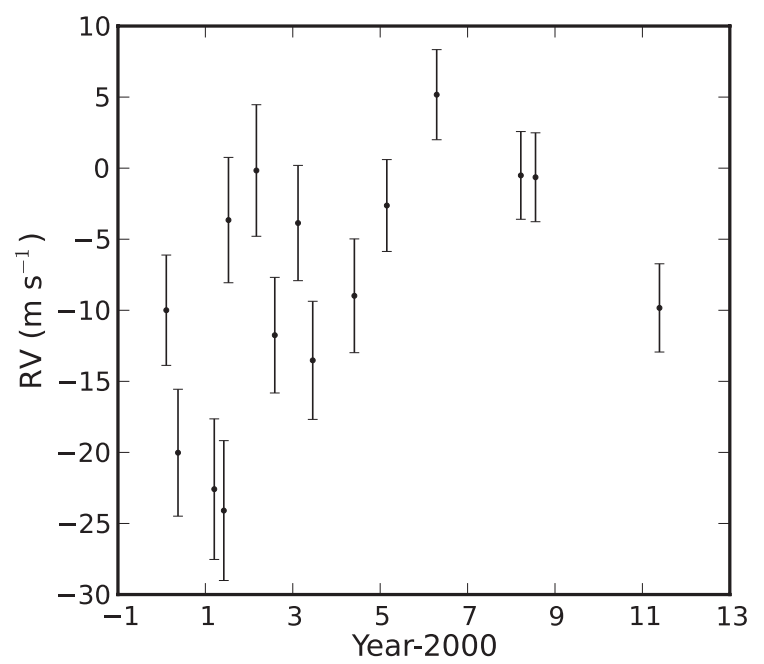

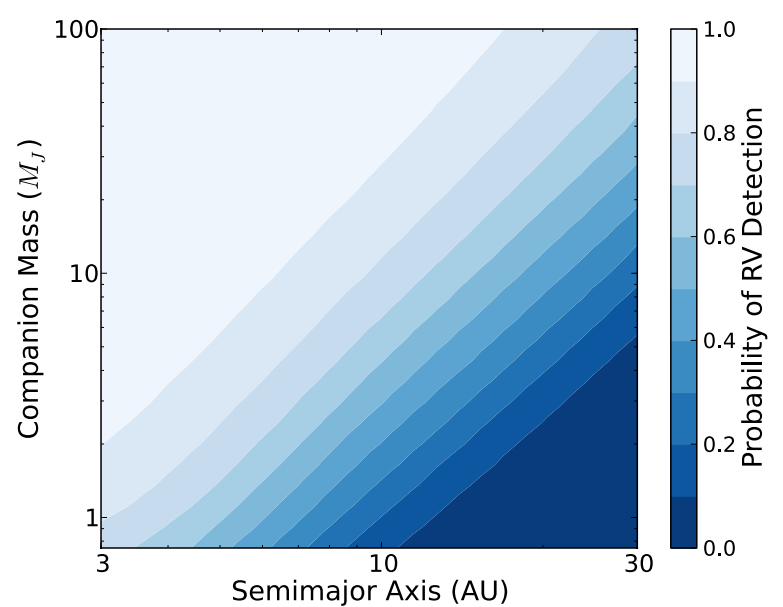

Figure 4. Ensemble average likelihood over all 111 stars of an RV detection for a companion to a star in our sample as a function of companion mass and orbital semimajor axis. We can detect accelerations induced by planets as small as $1 M_{J}$ in short orbits, but a planet distribution function is required to determine the number of $1 M_{J}$ planets in wide orbits and calculate the overall giant planet occurrence rate.

(A color version of this figure is available in the online journal.)

a circular orbit, the magnitude of the change in $\mathrm{RV}, \dot{\gamma}=d v / d t$, is given by

$$
\dot{\gamma}=6.57 \mathrm{~m} \mathrm{~s}^{-1} \mathrm{yr}^{-1}\left(\frac{m_{p}}{M_{J}}\right)\left(\frac{a}{5 \mathrm{AU}}\right)^{-2} \hat{v}_{p} \cdot \hat{r}_{\mathrm{los}},
$$

with $M_{J}$ the mass of Jupiter and $a$ the orbital semimajor axis. $\hat{v}_{p}$ and $\hat{r}_{\text {los }}$ are unit vectors along the direction of the planet's velocity vector and the line of sight, respectively. When the companion has longitude of periapsis $\varpi=90$ or 270 , the magnitude of this trend is maximized: $\hat{v}_{p} \cdot \hat{r}_{\mathrm{los}}=\sin i$. To determine if our observed accelerations are caused by planets or more massive companions, we obtained AO imaging observations of each star.

\subsection{Adaptive Optics Observations}

The detectability diagnostics developed in Section 2.2 are based strictly on the information encoded in the RV data. Since we are looking at accelerations caused by objects in wide orbits

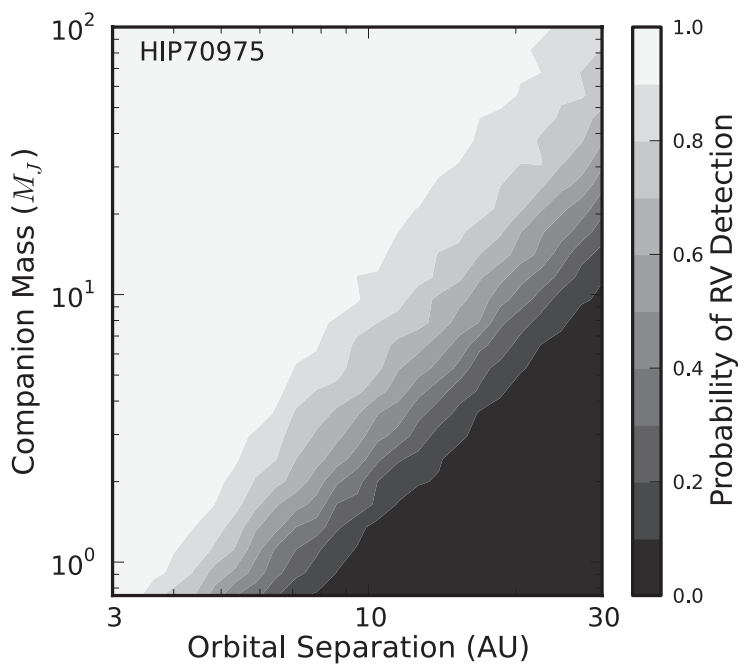

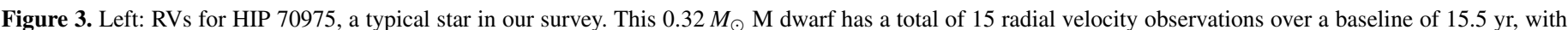

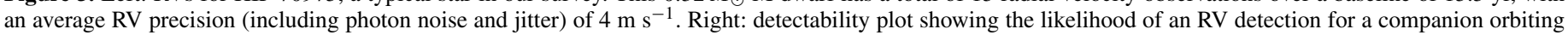
HIP 70975 as a function of companion mass and semimajor axis from its host star. 


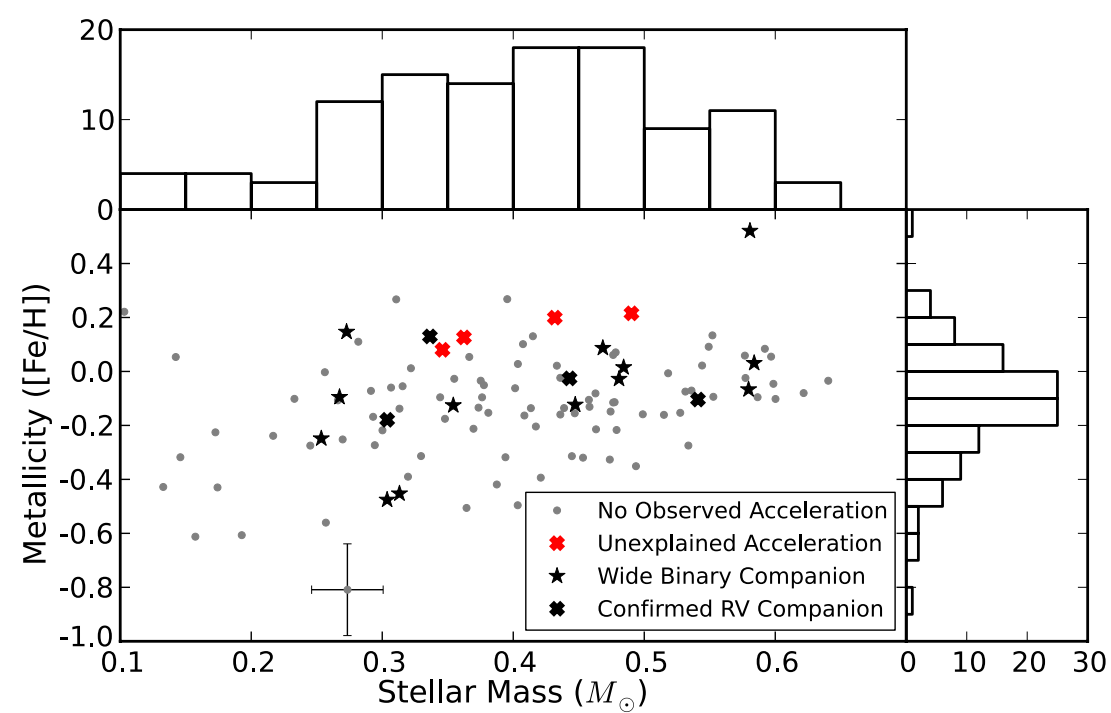

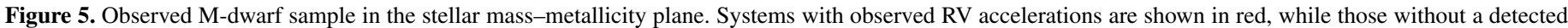

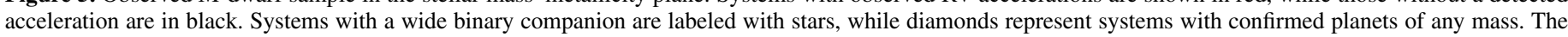
error bars displayed for HD 33793 are representative of the uncertainties for all stars in our sample.

(A color version of this figure is available in the online journal.)

Table 3

Previously Published RV Planets

\begin{tabular}{lcccc}
\hline \hline Star & $\begin{array}{c}\text { Planet } m \text { sin } i \\
\left(M_{J}\right)\end{array}$ & $\begin{array}{c}\text { Period } \\
\text { (days) }\end{array}$ & Discovery & Updated Parameters \\
\hline Gl 179 & $0.82 \pm 0.07$ & $2288 \pm 59$ & Howard et al. (2010a) & Howard et al. (2010a) \\
Gl 317 & $1.80 \pm 0.05$ & $691.8 \pm 4.7$ & Johnson et al. (2007) & Anglada-Escudé et al. (2012b) \\
Hip 57050 & $0.298 \pm 0.025$ & $41.397 \pm 0.016$ & Haghighipour et al. (2010) & Haghighipour et al. (2010) \\
Gl 436 & $0.0737 \pm 0.0052$ & $2.643899 \pm 0.000001$ & Butler et al. (2004) & Southworth (2010) \\
Gl 581 & $0.049 \pm 0.001$ & $5.369 \pm 0.002$ & Bonfils et al. (2005) & Tadeu dos Santos et al. (2012) \\
& $0.017 \pm 0.001$ & $12.931 \pm 0.002$ & Udry et al. (2007) & Tadeu dos Santos et al. (2012) \\
& $0.006 \pm 0.003$ & $1.0124 \pm 0.0001$ & Udry et al. (2007) & Tadeu dos Santos et al. (2012) \\
Gl 649 & $0.006 \pm 0.003$ & $2.149 \pm 0.002$ & Mayor et al. (2009) & Tadeu dos Santos et al. (2012) \\
Gl 849 & $0.328 \pm 0.032$ & $598.3 \pm 4.2$ & Johnson et al. (2010b) & Johnson et al. (2010b) \\
Gl 876 & $0.82 \pm 0.07$ & $1890 \pm 130$ & Butler et al. (2006) & Butler et al. (2006) \\
& $1.9506 \pm 0.0039$ & $61.1166 \pm 0.0086$ & Marcy et al. (1998) & Rivera et al. (2010) \\
& $0.612 \pm 0.003$ & $30.0881 \pm 0.0082$ & Marcy et al. (2001) & Rivera et al. (2010) \\
& $0.018 \pm 0.001$ & $1.93778 \pm 0.00002$ & Rivera et al. (2005) & Rivera et al. (2010) \\
& $0.039 \pm 0.005$ & $124.26 \pm 0.70$ & Rivera et al. (2010) & Rivera et al. (2010) \\
\hline
\end{tabular}

around the primary star, we must break the degeneracy between companion mass and orbital semimajor axis for a given observed acceleration. AO imaging allows us to immediately detect the presence or nonexistence of nearly all stellar-mass companions and most brown dwarf companions to our primary stars, so we can readily separate stellar-induced accelerations from those caused by planets.

All four targets with an observable RV acceleration were observed with NIRC2 (instrument PI: Keith Matthews) at the W.M. Keck Observatory using the AO system (Wizinowich et al. 2000; Table 4). In most cases, images were obtained in the $K^{\prime}$ filter $\left(\lambda_{c}=2.12 \mu \mathrm{m}\right)$. We nominally execute a three-point dither pattern to facilitate removal of instrument and sky background noise. Images were processed by flat-fielding, correcting for hot pixels with interpolation, subtracting the sky background, and rotating the frames to standard northeast orientation. In three cases, we applied the angular differential imaging (ADI) point spread function subtraction technique, allowing the observed field to rotate around the target star during the observation, while instrumental artifacts remain fixed. In all cases, we use the large hexagonal pupil mask and the narrow camera. For all four systems exhibiting long-term RV accelerations, we did not image a massive companion. In the cases where our field of view is not large enough to eliminate the possibility of massive stars in very wide orbits $\left(>4^{\prime \prime}\right)$, we supplement our AO data with publicly available 2MASS images.

The luminosity ratio between our $\mathrm{M}$ dwarfs and their companions depends on the mass of the companion and the age of the system. Stars observed by the CPS team are selected to avoid excessive chromospheric activity, and are thus likely older than 1 Gyr (Wright 2005). We assume all targets have fully contracted and assert an age of 5 Gyr for each system. For systems with nondetections, we estimate the flux (and thus the mass) a companion would need to have to be observed at a given projected separation in our observations. From that value, we can then determine the region of parameter space excluded by the observations (Figure 7). In general, AO imaging eliminates nearly all stellar companions, while ADI can also probe the brown dwarf mass regime.

For each of our targets with unexplained accelerations, a contrast curve showing the mass to which we are sensitive to companions at the $5 \sigma$ level as a function of projected separation 

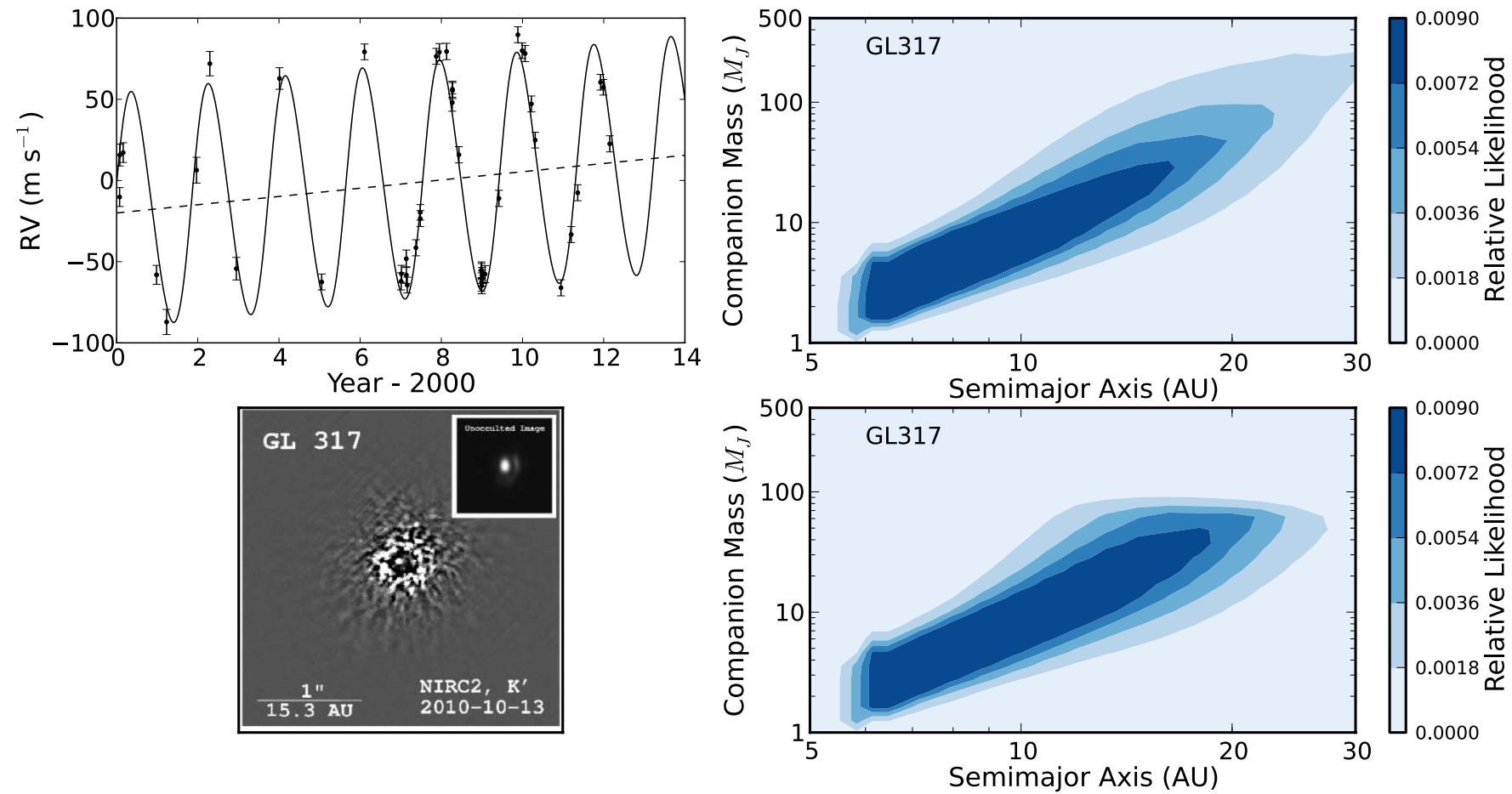

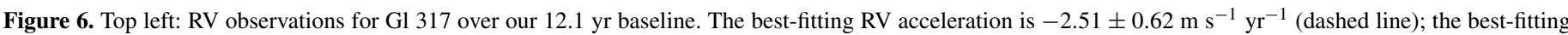

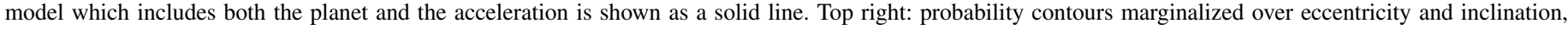

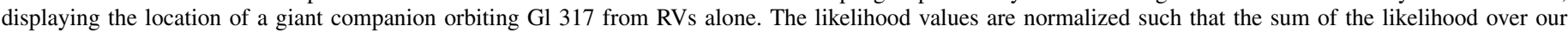

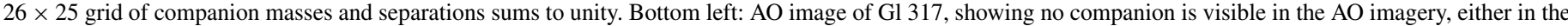

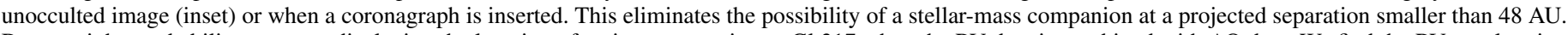

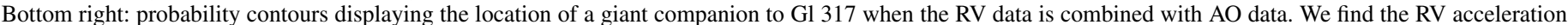
is likely induced by a substellar companion.

(A color version of this figure is available in the online journal.)

Table 4

Stars with Measured RV Accelerations and Imaging Nondetections

\begin{tabular}{|c|c|c|c|c|c|c|}
\hline Star & $\begin{array}{c}\text { RV Slope } \\
\left(\mathrm{m} \mathrm{s}^{-1} \mathrm{yr}^{-1}\right)\end{array}$ & AO Observation Date & Instrument & Filter & ADI & Cause of Acceleration \\
\hline Gl 317 & $2.51 \pm 0.62^{\mathrm{a}}$ & 2010 Oct 13 & NIRC2 & $K^{\prime}$ & Yes & Presumed companion \\
\hline Gl 179 & $-1.17 \pm 0.29$ & 2012 Feb 2 & NIRC2 & $K^{\prime}$ & Yes & Presumed companion \\
\hline Hip 57050 & $1.39 \pm 0.39$ & 2012 Dec 27 & NIRC2 & $K s$ & No & Presumed companion \\
\hline G1 849 & $\mathrm{~N} / \mathrm{A}^{\mathrm{b}}$ & 2011 Jun 24 & NIRC2 & $L$ & Yes & Identified companion \\
\hline Hip 63510 & $\mathrm{~N} / \mathrm{A}^{\mathrm{b}}$ & $\mathrm{N} / \mathrm{A}$ & $\mathrm{N} / \mathrm{A}$ & $\mathrm{N} / \mathrm{A}$ & $\mathrm{N} / \mathrm{A}$ & Brown dwarf ${ }^{\mathrm{a}}$ \\
\hline Hip 71898 & $8.6 \pm 0.4$ & $\mathrm{~N} / \mathrm{A}$ & $\mathrm{N} / \mathrm{A}$ & $\mathrm{N} / \mathrm{A}$ & $\mathrm{N} / \mathrm{A}$ & Brown dwarf ${ }^{c}$ \\
\hline
\end{tabular}

Notes.

${ }^{a}$ Beuzit et al. (2004).

${ }^{\mathrm{b}}$ Curvature in RV.

c Golimowski et al. (2004).

is shown in Figure 7. This choice provides similar results to the detection limits found by visual inspection, as tested by injecting artificial companions into AO images (Metchev \& Hillenbrand 2009). We convert relative brightness to mass using the theoretical evolutionary tracks of Baraffe et al. (2003) for substellar companions and Girardi et al. (2002) for more massive companions. Interpolation between the two sets of models provides reasonable results in the intermediate domain near $125 M_{J}$. The resultant parameter space where a companion could reside for G1 317 to cause the observed stellar acceleration is shown in Figure 6.

The assumption of a $5 \mathrm{Gyr}$ age for each star does not significantly affect our results. For all plausible system ages, stellar-mass companions would be easily detectable by AO. Our sensitivity to stars is independent of assumed age, as luminosities of $\mathrm{M}$ dwarfs are constant over the age of the universe. At no ages $>1$ Gyr are we sensitive to any planetary mass companions with AO. As shown in Figure 8, assuming a different age for each star would only change the efficiency of detecting brown dwarfs. Since the occurrence rate of brown dwarfs is only a few percent, much smaller than the occurrence rate of planets or low-mass stars (Metchev \& Hillenbrand 2009; Dieterich et al. 2012), errors induced by assuming an incorrect stellar age from missed brown dwarfs are negligable. "False negatives" such as these will be discussed in Section 3.1.

\section{MEASURING THE GIANT PLANET OCCURRENCE RATE}

We estimate the occurrence rate of giant planets orbiting $\mathrm{M}$ dwarfs using statistical inference. The fraction of stars which 


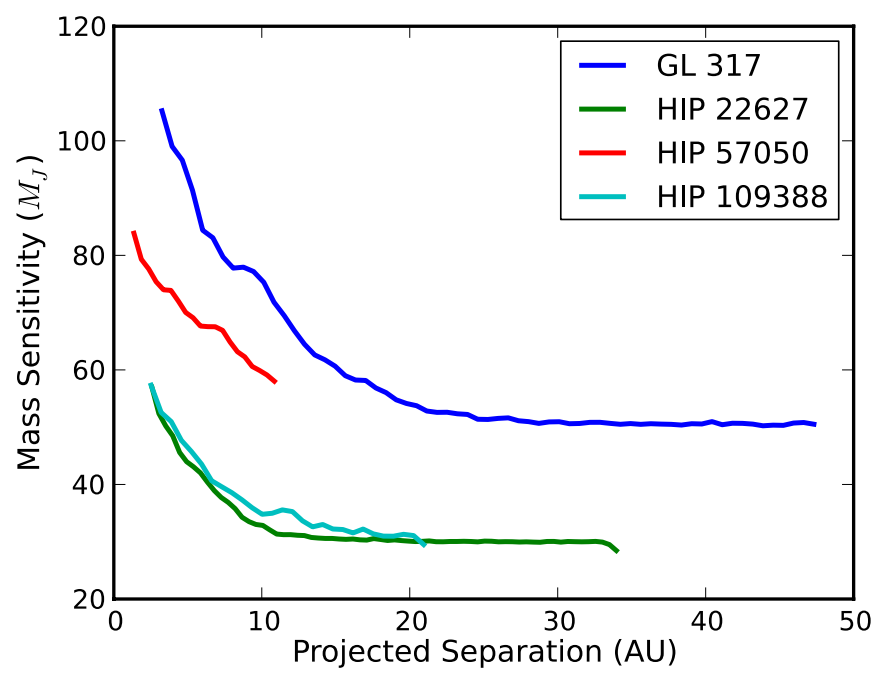

Figure 7. Mass sensitivity for a $5 \sigma$ detection of a companion object as a function of projected angular separation for each of our four stars with long-term RV drifts. The maximum projected separation eliminated corresponds to the field of view of the AO system and thus varies for each star as a function of the distance to each star. For all stars except HIP 57050, we rule out stellar-mass companions beyond 1 arcsec through our AO imaging. When our field of view is small, we supplement our AO data with 2MASS seeing-limited images. Stellar companions at small projected separations would have RV accelerations larger than those observed in our sample.

(A color version of this figure is available in the online journal.)

host giant planets, given some number of observed accelerations, $N$ trends, and some number of nondetections, $N$ ND, from a sample of targets, is given such that

$$
f_{\mathrm{pl}}=\frac{N_{\text {trends }} P(\text { planet } \mid \text { trend })+N_{\mathrm{ND}} P(\text { planet } \mid \mathrm{ND})}{N_{\text {targets }}} .
$$

To calculate the posterior probability that a given star hosts a gas giant planet, we must estimate the a priori likelihood that a planet exists given the presence of an RV acceleration (a true positive), the likelihood a planet would not be detected in an RV survey (a false negative) and the likelihood that an observed acceleration is caused by some effect other than the movement of a planet (a false positive).

\subsection{False Negatives}

There are multiple ways for a giant planet to be missed in our survey. For each planet in a wide orbit, we observe only a fraction of a revolution. A planet near its maximal sky-projected separation from its host star has acceleration primarily in the tangential, not radial, direction. In cases such as this the change in RV over our observing baseline may not be noticeable. Thus we may expect to have a lower RV detection efficiency for planets near their maximal sky-projected separation.

Similarly, we may expect to have a lower imaging detection efficiency for stars near their minimal sky-projected separation, when the RV acceleration is the largest. However, in these cases we would still expect to detect the binary companion. If the companion is located directly along the line of sight to the star, then it will also appear in the $0{ }^{\prime \prime} 85$ spectrograph slit used with HIRES. Therefore, we would expect such systems to appear as SB2s. We explore this fully in Section 4.2 and show that we would detect all such systems.

To determine the likelihood that such a planet would be missed by our search, we use our detectability matrices developed in Section 2.2. We assume the distribution of planets

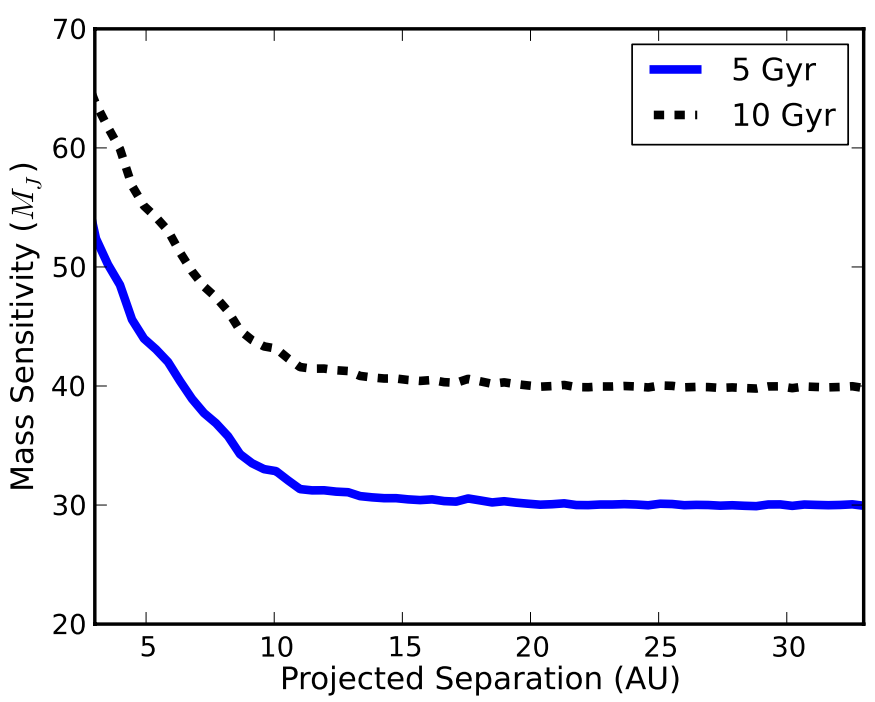

Figure 8. Adaptive optics mass exclusion plot for the star HIP 22627 showing the relative insensitivity of our results to the assumed age of M-star planet hosts. AO observations rule out essentially all stellar-mass companions. Sensitivity to substellar objects is a function of age, but brown dwarfs are scarce at close separations (Marcy \& Butler 2000), and wide separations (Metchev \& Hillenbrand 2009). Thus, our estimate of the planet frequency around M stars is only weakly dependent on our assumed age of the host stars.

(A color version of this figure is available in the online journal.)

follows a double power law, such that

$$
\frac{d^{2} N}{d \log m d \log a} \propto m^{\alpha} a^{\beta},
$$

similar to that assumed by Cumming et al. (2008) and Bowler et al. (2010), and comparable to the power-law distributions applied in the analyses of microlensing surveys. At a given companion mass and semimajor axis, we can then determine the relative likelihood that a planet exists at this position. We multiply this by the likelihood of detecting such a planet to determine the fraction of planets we would find orbiting each star and the fraction we would miss. These numbers are determined through our analysis of observations of simulated injected planets, as developed in Section 2.1.

We can test our detectability calculation by analyzing the known wide-separation companions in our sample. Of our 111 stars, four are known to host directly imaged brown dwarf companions (see Appendix A.4-A.7). Of these, two (HD 71898B and HIP 63510B) were detected as accelerations in our sample, while two (Gl 569B and Gl 229B) are at very large separations and were not detected. The detection or nondetection of each system is consistent with what would be expected from our analysis of injected planets (see Appendix A.4-A.7).

We detect the two brown dwarfs with high expected RV detection efficiency, and do not detect the two with expected detection efficiencies near zero, both of which have $a>$ 40 AU. We would like a larger sample to test this method, but the limited number of brown dwarfs suggests our ability to detect giant planets is consistent with expectations. This sample also suggests $f_{\mathrm{BD}}$ is only a few percent, consistent with complementary studies (Dieterich et al. 2012).

A giant planet could also be missed if it was in a system with multiple giant planets. We observe only the sum of all RV signals from all planets orbiting a star. For example, if a star hosts two giant long-period planets with one on each side of the star, the two signals would destructively interfere. Even 
if the acceleration was still detectable, this interference would cause us to measure an incorrect magnitude of the acceleration, so our probability contours would be incorrect. Giant planet multiplicity around $\mathrm{M}$ dwarfs is not well understood, but since giant planet occurrence is believed to be small (Bonfils et al. 2013) the multiplicity rate of giant planets around $\mathbf{M}$ dwarfs is likely also small. Presently, there are no known systems with two planets larger than Jupiter orbiting one $M$ dwarf. Even in cases with two large planets, one planet will dominate the RV signal. For example, OGLE-2006-BLG-109L contains a $0.73 \pm 0.06 M_{J}$ planet at $2.3 \pm 0.5 \mathrm{AU}$ and a $0.27 \pm 0.02 M_{J}$ planet (slightly less massive than Saturn) at $4.5_{-1.0}^{+2.1}$ AU (Gaudi et al. 2008; Bennett et al. 2010). In this case, the Doppler amplitude of the inner planet would be a factor of 3.3 larger than the Doppler amplitude of the outer planet. Similarly, an external observer of the solar system would observe an RV signal from Jupiter 4.5 times larger than that of Saturn. Thus we neglect this possible source of error.

We then claim that the likelihood of the existence of a giant planet given the nondetection of an RV acceleration is

$$
P(\text { planet } \mid \mathrm{ND})=f_{\mathrm{pl}}\left(1-\eta_{\mathrm{pl}, \star}\right),
$$

where $\eta_{\mathrm{pl}, \star}$ is the probability of detecting a giant planet around a given star as a function of planet mass and orbital semimajor axis, estimated by simulating observations of injected planets. The true probability of missing a planet depends on the true giant planet occurrence rate and the planet distribution function. We can determine this value directly if the underlying planet distribution function (Equation (7)) is assumed. By counting the observed trends and analyzing our RV detection efficiencies for each star as a function of mass and separation, we can determine the number of missed planets. We find our final result is not a strong function of mass index $\alpha$ or semimajor axis index $\beta$ (see Section 4.7).

\subsection{False Positives}

\subsubsection{Multiple Planets}

In some cases, observed accelerations may not be induced by the orbit of a giant planet. If two smaller planets are orbiting one star, when they are both on the same side of the star their RV signals would constructively interfere, giving the appearance of a giant planet where none exists. Again, multiplicity rates of large planets are unknown for these small stars but are likely small; we again neglect this effect as a possible source of error. This is a reasonable assumption even if the multiplicity rate of gas giant planets around $\mathrm{M}$ dwarfs was much larger than currently expected. Both the orientation of the system and the relative positioning of the planets during our observations is random. Therefore, it is equally likely that multiple planets would be in the "constructive" or "destructive" phase of their orbits. Thus, similar numbers of false additional planets would be added to our sample as missed true planets.

\subsubsection{Secular Acceleration}

A false positive can also be caused by secular acceleration. When a high proper motion star moves quickly relative to the Sun, its peculiar velocity vector changes direction in time, causing the star's systemic RV to increase. For a star with proper motion $\mu$ at a distance $d$ the magnitude of this effect is, to first order,

$$
\dot{\gamma}=23.0 \mathrm{~cm} \mathrm{~s}^{-1} \mathrm{yr}^{-1}\left(\frac{d}{10 \mathrm{pc}}\right)\left(\frac{\mu}{1 \operatorname{arcsec} \mathrm{yr}^{-1}}\right)^{2} .
$$

The secular acceleration $\dot{\gamma}$ is always positive, so that the star's RV only increases because of this effect. For several nearby stars secular acceleration is large enough to create an apparent acceleration or cause an astrophysical RV acceleration to be incorrectly measured. For example, Barnard's star has a secular acceleration of $4.515 \pm 0.002 \mathrm{~m} \mathrm{~s}^{-1} \mathrm{yr}^{-1}$ (Choi et al. 2013), larger than all of our observed accelerations. Fortunately, the magnitude of the secular acceleration can be precisely quantified if the star's distance and proper motion are known. All of our stars have measured proper motions and parallaxes, so we can determine the expected secular contribution. This acceleration is subtracted from the observed RV automatically by the CPS RV pipeline (Howard et al. 2010a), so this potential source of error is automatically accounted for in our data. Moreover, none of our observed accelerations are consistent with what would be expected from secular acceleration alone.

\subsubsection{Magnetic Activity}

Magnetic activity on a star can cause a false positive: rotating active regions can affect the shape of the observed spectral lines and thus the apparent RV (Gray 1988). A magnetic cycle can occur over years and hide or mimic a RV signal. We denote the fraction of stars with a magnetically induced acceleration as $f_{A}$. Gomes da Silva et al. (2012) claim six stars from their sample of $27 \mathrm{M}$ dwarfs with variability $(22 \% \pm 9 \%)$ have RVs induced by magnetic activity. We are interested in the converse (how many trends are induced by variability?) but their result suggests $f_{A}$ may be significant. To determine $f_{A}$, we review all $165 \mathrm{M}$ dwarfs observed by the CPS team, both as part of this survey and as part of the M2K survey (Apps et al. 2010; Fischer et al. 2012). Between these two programs, there are a total of 34 systems with RV trends. We analyze the $S_{\mathrm{HK}}$ values for these stars and find the RV correlates with $S_{\mathrm{HK}}$ with a correlation coefficient $|r|>0.5$ in 7 cases, suggesting $20.6 \% \pm 7.8 \%$ of long-term RV trends may be magnetically induced. We adopt this value as $f_{A}$. Even if the true value for $f_{A}$ is a factor of two larger, it would decrease our planet occurrence rate from $f_{\mathrm{pl}}=6.5 \%$ to only $f_{\mathrm{pl}}=4.9 \%$, still within our uncertainties.

\subsubsection{Brown Dwarfs}

Our AO search is sensitive to all stellar-mass companions, but only to the most massive brown dwarfs. We can detect brown dwarfs larger than approximately $50 M_{J}$, although this number varies from target to target. For each target, we can determine the fraction of brown dwarfs we would expect to detect by our AO imaging, given the assumption that a trend was caused by a brown dwarf. We call this efficiency $\eta_{\mathrm{BD}}$. Here, we assume a form for the brown dwarf mass function where $d n / d \log (m) \propto m^{0.4 \pm 0.2}$ (Peña Ramírez et al. 2012). Thus we can estimate the likelihood of detecting a brown dwarf around a star in our sample, given that a brown dwarf exists. To estimate the probability a brown dwarf exists, we use the result of Dieterich et al. (2012), who, through an HST/NICMOS snapshot program, estimate that $f_{\mathrm{BD}}=2.3_{-0.7}^{+5.0} \%$ (at $1 \sigma$ ) of $\mathrm{M}$ dwarfs have an $\mathrm{L}$ or $\mathrm{T}$ companion between 10 and $70 \mathrm{AU}$. This is consistent with the result of Metchev \& Hillenbrand (2009), who estimate a brown dwarf companion frequency of $f_{\mathrm{BD}}=3.2_{-2.7}^{+3.1} \%($ at $2 \sigma)$ around solar-type (FGK) stars.

\subsubsection{White Dwarfs}

Compact stellar remnants are often faint and such binary companions can evade direct detection, especially when the 
compact object is cool $(T<4000 \mathrm{~K})$ so that the infrared light is dominated by the primary star (Crepp et al. 2013a). Since our targets are all $\mathrm{M}$ dwarfs, it is not unreasonable to expect that some may have formed as lower-mass companions in binary systems with the higher-mass object having evolved off the main sequence to become a white dwarf. Napiwotzki (2009) combine observations of local white dwarfs with galactic structure models and find that in the thin disk, there is a white dwarf number density of $n_{\mathrm{WD}}=2.9 \times 10^{-3} \mathrm{pc}^{-3}$. From an analysis of PanSTAARS data, Wheeler (2012) estimate $20 \%$ of all white dwarfs have an M-dwarf companion $\left(f_{M \mid \mathrm{WD}}\right)$, somewhat larger than the $12 \%$ found by Napiwotzki (2009). Considering the measurement by Chang et al. (2011) of $n_{\star}=0.030 \pm 0.002$ stars $\mathrm{pc}^{-3}$, and that approximately $70 \%$ of all stars are $\mathrm{M}$ dwarfs $\left(f_{M \mid \star}\right.$; Henry et al. 2006), we can determine the fraction of $M$ dwarfs in the thin disk with a white dwarf companion, a number we define as $f_{\mathrm{WD}}$. If we take $f_{M \mid \mathrm{WD}}=0.16 \pm 0.04$, we find that

$$
f_{\mathrm{WD}}=\frac{n_{\mathrm{WD}} f_{M \mid \mathrm{WD}}}{n_{\star} f_{M \mid \star}}=2.2 \% \pm 0.5 \%,
$$

where the error is dominated by the uncertainty in $f_{M \mid \mathrm{WD}}$.

By combining the false positive events from Sections 3.2.3 to 3.2 .5 , we conclude that given the existence of a trend in our data, the likelihood it is caused by a giant planet is

$$
\begin{aligned}
P(\text { planet } \mid \text { trend })=\left(1-f_{A}\right)\left[1-f_{\mathrm{BD}}\left(1-\eta_{\mathrm{BD}, \star}\right)\right]\left(1-f_{\mathrm{WD}}\right) . \\
\text { 3.3. Determining } f_{\mathrm{pl}}
\end{aligned}
$$

We determine the giant planet occurrence rate, $f_{\mathrm{pl}}$ by combining our estimate of the number of false positives and false negatives with the number of observed accelerations. Specifically, the occurrence of giant planets is given by Equation (6) if the number of observed accelerations is known, along with the probability of a false negative or false positive in our sample. These probabilities are defined by Equations (8) and (11), respectively.

For each star in our sample, we use our map of giant companion detectability (e.g., Figure 6) to estimate our efficiencies, $\eta_{\mathrm{BD}}$ and $\eta_{\mathrm{pl}}$. We measure the total planet fraction, $f_{\mathrm{pl}}$ and its uncertainty through a Monte Carlo experiment. For each trial, we establish an expected number of observed accelerations, drawing from a binomial distribution with $n=111$ and $p=4 / 111$, representing the most likely underlying distribution behind our observed sample. In practice, we draw from our star list 111 times, with replacement, to determine a stellar sample. We then draw randomly from our previously defined distributions to estimate $f_{A}, f_{\mathrm{BD}}$ and $f_{\mathrm{WD}}$. These values are sufficient to calculate the probability an observed acceleration is caused by a false positive astrophysical event. In cases where known planets with masses $m>1 M_{J}$ exist in our sample, we include their presence in our calculation of $f_{\mathrm{pl}}$.

The derivative of the RV acceleration (the "jerk") for Gl 849 is nonzero, so we can use the RV information to fit a twoplanet model to this system, instead of a planet plus a linear acceleration (see Appendix A). We find the inner planet to have a mass $m \sin i=0.90 \pm 0.05 M_{J}$ with a period of $5.24 \pm 0.07 \mathrm{yr}$, and the outer planet to have a mass $m \sin i=0.70 \pm 0.31 M_{J}$ with a period of $19.3_{-5.9}^{+17.1} \mathrm{yr}$. More data is needed to determine the exact parameters of the orbit of Gl 849c, but from the existing $\mathrm{RV}$ information we can determine the probability each planet has a mass $m>1 M_{J}$. The exact value depends on the planet

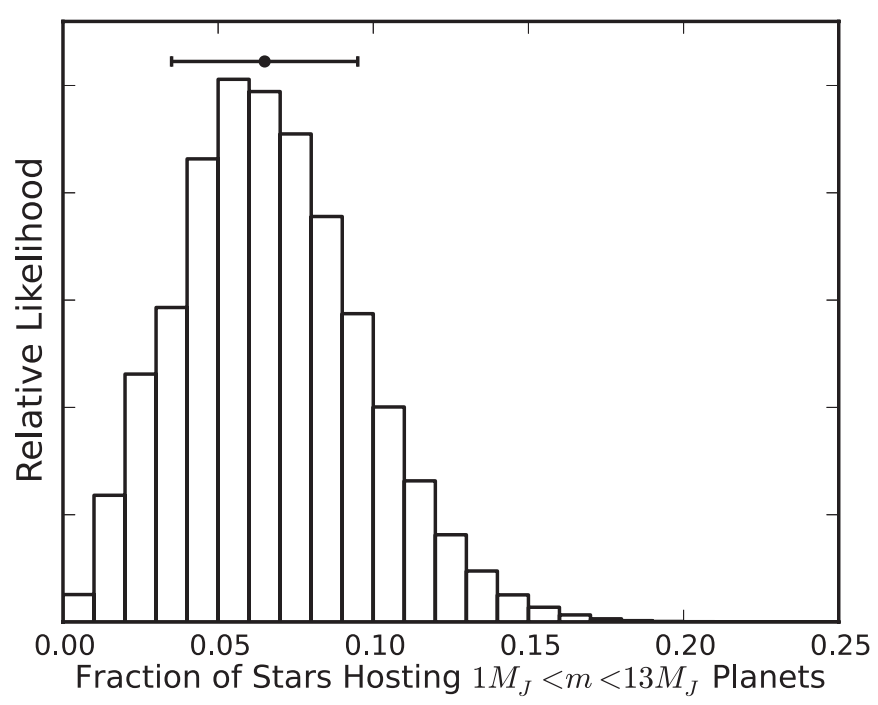

Figure 9. Giant planet occurrence for our sample of 111 nearby $\mathrm{M}$ dwarfs. We find $6.5 \% \pm 3.0 \%$ of $\mathrm{M}$ dwarfs host a planet with mass $1 M_{J}<m<13 M_{J}$ and $0<a<20 \mathrm{AU}$.

mass distribution function; assuming each orientation has equal probability (so that $\alpha=-1$ ) we find probabilities of 0.577 and 0.419 , respectively. Following the method of Ho \& Turner (2011), we find changing the distribution function changes these values by less than $10 \%$.

Since we know the region of mass-separation parameter space to which we are sensitive to planets for each star, we can self consistently estimate the planet frequency in this parameter space. We then assume the result from Cumming et al. (2008), who find the power-law indices (Equation (7)) of $\alpha=-0.31 \pm 0.20$ and $\beta=0.39 \pm 0.15$. We randomly select values for $\alpha$ and $\beta$ from these distributions and use our detection efficiencies to determine the number of false negative missed planets in our sample. Through Equation (6), we then have enough information to estimate the planet fraction as a function of each parameter. By repeating this process many times, varying each of our assumed parameters, we can measure the overall planet fraction and its uncertainty.

\section{RESULTS AND DISCUSSION}

\subsection{The Frequency of Giant Planets}

Given an observed trend, we can estimate the likelihood the signal is caused by a massive planet. By analyzing our 111 targets as described in Section 3.3, we recover a distribution in giant planet occurrence as shown in Figure 9. We find from this analysis that $6.5 \% \pm 3.0 \%$ of all $\mathrm{M}$ dwarfs host a giant planet with a semimajor axis smaller than $20 \mathrm{AU}$. This number is lower than previous studies of higher-mass stars. Bowler et al. (2010) find $24_{-7}^{+8} \%$ of "retired" A stars host Jupiter-mass planets within 3 AU, while Cumming et al. (2008) find that $f_{\mathrm{pl}}=10 \% \pm 1 \%$ of FGK stars host Jupiter-mass planets within $20 \mathrm{AU}$.

If we consider multiplicity in situations where we have a giant planet and an RV acceleration (or in the case of Gl 849, two giant planets), then we measure a giant planet occurrence rate of $0.083 \pm 0.019$ giant planets per star. To estimate this, we repeat the calculations of the previous section, but count known giant planets separate from observed accelerations in the cases when we observe both a planet and a "trend." This number does implicitly assume that observed accelerations are caused by the motion of one giant planet, not a combination of multiple 
planets in motion. The multiplicity rate of giant planets around $\mathrm{M}$ dwarfs appears to be lower than the multiplicity rate of small planets, such as those detected by Kepler (Youdin 2011).

Our result is consistent with the result of microlensing surveys of $\mathrm{M}$ dwarfs, which suggest a total occurrence rate of $0.09_{-0.05}^{+0.03}$ giant planets per star in the range $1 M_{J}<m<10 M_{J}$ and $0.5 \mathrm{AU}<a<20 \mathrm{AU}$ Cassan et al. (2012). However, the power-law distribution determined by the microlensing studies is considerably different than the Cumming et al. (2008) distribution assumed here. We discuss this further and constrain $\alpha$ and $\beta$ in Section 4.8.

This is the first study using observed RV accelerations to estimate the giant planet occurrence rate. However, previous $\mathrm{RV}$ studies have discussed the presence or nondetection of RV accelerations in their analysis. For example, Endl et al. (2003) mentioned all RV accelerations in their sample are likely the cause of stellar binaries. Our observations are generally more precise than theirs, as we detect some planets that they miss (such as Gl 436 and Gl 849).

Bonfils et al. (2013) detect 15 long-term accelerations in their sample of 102 southern M dwarfs. Some of these can be attributed to long period binary companions (such as Gl 250B and Gl 618A). Of the stars where we detect an RV acceleration, only one (Gl 849) is in the HARPS sample; these authors also detect an acceleration. Bonfils et al. (2013) also detect an acceleration around G1 699 (Barnard's star) that we do not detect. Such an acceleration has also not been found by other studies: Choi et al. (2013) claim the RV of Barnard's star is increasing at $4.515 \pm 0.002 \mathrm{~m} \mathrm{~s}^{-1} \mathrm{yr}^{-1}$, consistent with the expected secular acceleration but inconsistent with the $-3.043 \pm 0.646 \mathrm{~m} \mathrm{~s}^{-1} \mathrm{yr}^{-1}$ acceleration observed by Bonfils et al. (2013). With more observations over a longer time baseline, this discrepancy will be resolved.

\subsection{Potential Missed Binary Stars}

We only collect AO images for systems with long-term RV accelerations. For these accelerations to be observable, the orbiting companion must have a component of its movement along our line of sight so that the RV changes during an orbit. A giant planet would be missed if it was in a near face-on orbit, such that the star's reflex motion was primarily in the plane of the sky. Such systems are accounted for in our detectability calculations (Figure 3), as we have determined the probability of detecting a planet's RV acceleration as a function of its mass and separation, marginalized over all other orbital parameters. These calculations do not, however, account for the possible presence of close stellar binary companions in face-on orbits. Although less common than edge-on systems, any missed binary systems that we have not rejected from our sample would cause our planet occurrence rate to be artificially low (assuming these systems could not form dynamically stable planets). Close binaries would be observable as double-lined spectroscopic systems (SB2s) in the CPS data, while wider binary pairs would be easily imaged by AO systems.

The RV sample was originally selected to reject systems with known binary companions within 2 arcsec. We would expect companions with a flux ratio larger than $0.01(\Delta V=5)$ to be detected as binaries (Robinson et al. 2007). For our brightest targets, this would correspond to M6 dwarfs and brighter. As the cutoff for hydrogen burning is the M6 spectral class (Luhman 2012), we would expect all close stellar-mass binaries to be removed from our HIRES observations.
To determine how many missed binaries are in our sample, we simulate a population of binary companions to $\mathbf{M}$ dwarfs. We create binary companions such that their semimajor axes are assigned following the observed distribution found by Fischer \& Marcy (1992). We randomly assign the other orbital parameters and determine there is a $41.8 \% \pm 0.3 \%$ chance a binary companion in our sample around a random star would have a projected separation smaller than 2 arcsec. Thus, considering Fischer \& Marcy (1992) find $42 \% \pm 9 \%$ of local M dwarfs are in binary or multiple systems, we would expect to have a total of $24 \pm 6$ binary systems in our sample, which originally contained 137 stars before the removal of known binaries. As we actually observe 22 binary systems (containing 26 stars), this result is consistent with our expectation.

We then determine the RV each simulated binary star would induce on our host companion. For each binary that induces a measurable acceleration on the host star, we simulate imaging observations to determine the probability this binary companion would be detected in either our AO survey or, for very wide separation binaries, a seeing-limited ground based survey such as 2MASS. By applying our joint AO/seeing-limited contrast curves, we find that if a binary star system in our survey induces an RV acceleration, we would have a $96.0 \% \pm 0.4 \%$ chance of imaging this binary companion. Therefore the probability that one or more of our observed accelerations is caused by a "missed" binary companion is negligible and this possibility does not significantly affect our results.

\subsection{Dependence on Stellar Mass}

Previous RV studies have found a correlation between stellar mass and giant planet occurrence at $a<2.5 \mathrm{AU}$, with more massive stars more likely to host giant planets (Johnson et al. 2010a). To test this relation inside the M-dwarf spectral class, we analyzed the high-mass stars separately from the low-mass stars in our sample. From our best-fit masses, half of our sample is more massive than $M=0.41 M_{\odot}$. We thus use this value as a dividing line to separate our sample into two groups. Our masses have typical uncertainties of $10 \%$, so for each star, given its mass and uncertainty, we determine the probability it is larger or smaller than $0.41 M_{\odot}$ assuming normally distributed errors. We then use that value as a weighting factor to assign a probability for each individual star to reside in our high-mass or low-mass bin, and then repeat our analysis for each individual subsample.

We find an occurrence rate for the high-mass subsample of $4.8 \% \pm 3.3 \%$ and for the low-mass subsample of $7.9 \% \pm 4.2 \%$ (Figure 10). Johnson et al. (2010a) find planet occurrence is correlated with stellar mass such that $f_{\mathrm{pl}} \propto$ $10^{(1.2 \pm 0.2)[\mathrm{Fe} / \mathrm{H}]} M_{\star}^{(1.0 \pm 0.3)}$. The average star in our high-mass sample has a mass of $0.5 M_{\odot}$ while the average star in our low-mass sample has a mass of $0.3 M_{\odot}$, so we would expect the high-mass subsample to have an occurrence rate larger than the low-mass sample by a factor of 1.67 . We find the true occurrence rate to change by a factor of $0.61 \pm 0.87$ in moving from the lower-mass to higher-mass bin. This is inconsistent with the expected result from Johnson et al. (2010a), but the difference between the two bins is not significantly different from zero. A larger sample is required to determine if the small difference between these two populations of $\mathrm{M}$ dwarfs is real or the result of a statistical anomaly. However, our result is lower than the Cumming et al. (2008) result for FGK stars, that $f_{\mathrm{pl}}=10 \% \pm 1 \%$ of FGK stars host Jupiter-mass planets within 20 AU. This difference is consistent with the Johnson et al. (2010a) correlation between stellar mass and planet occurrence. 


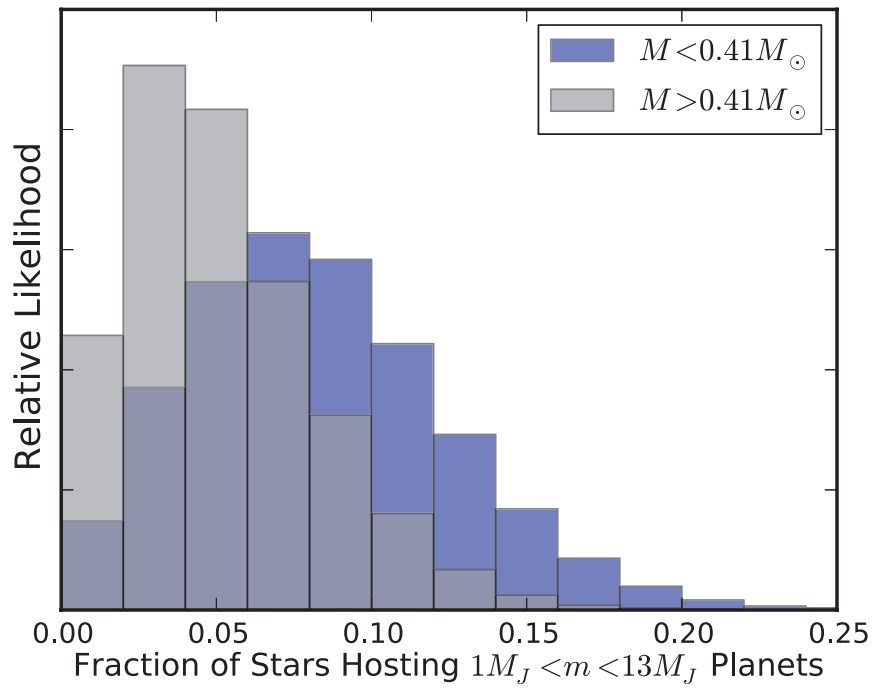

Figure 10. Planet occurrence for a low-mass subsample (blue) and a high-mass subsample (gray) of M dwarfs. Both subsets have nearly similar giant planet occurrence rates, suggesting planet occurrence may not depend strongly on stellar mass within the $\mathrm{M}$ spectral class. A larger sample is required to determine if the lack of difference in occurrence rates is astrophysical or statistical variance.

(A color version of this figure is available in the online journal.)

\subsection{Dependence on Metallicity}

Previous RV studies of giant planets have also found evidence for a correlation between planet occurrence and metallicity (Fischer \& Valenti 2005; Johnson et al. 2010a). To test if this correlation holds for more distant planets, we again split our sample into two, using the same method from the previous section. In this case, we use $[\mathrm{Fe} / \mathrm{H}]=-0.10$, the sample median metallicity, as the dividing line for our subsamples. We assume all stars have metallicity uncertainties of $0.17 \mathrm{dex}$, consistent with the scatter expected from the Neves et al. (2012) empirical relation. Again, we assume Gaussian errors to determine the probability each star is in a specific subsample. We then repeat our analysis on both groups.

In the high-metallicity subsample, we find an occurrence rate such that $12.4 \% \pm 5.4 \%$ of M-dwarfs host giant planets. In the low-metallicity sample the occurrence rate drops to $0.96 \% \pm 0.51 \%$. In Figure 11 we plot a histogram of our posterior distribution of planet occurrence for our high-metallicity subsample. Vertical lines represent (from left to right) $1 \sigma$ and $3 \sigma$ upper limits on the planet occurrence rate for the low-metallicity subsample. From these distributions, the giant planet occurrence rate for metal-rich stars has only a $2.4 \%$ probability of being lower than the $3 \sigma$ upper limit on the planet occurrence rate for metal-poor stars. The difference between these subsamples may be suggestive of the same effect seen for RV-confirmed planets within 2.5 AU (Johnson \& Apps 2009; Johnson et al. 2012).

An increase in the planet occurrence rate with metallicity for planets beyond a few AU may suggest giant planets in wide orbits are commonly formed by the same processes as the RV giant planet population. This study will be facilitated by the development of reliable spectroscopic metallicity measurements (Rojas-Ayala et al. 2010).

\subsection{The Stellar Mass-Metallicity Plane}

We can quantify our giant planet occurrence rate with respect to stellar mass and metallicity. Such an approach has been undertaken for planets with $a<2.5$ AU orbiting stars of all

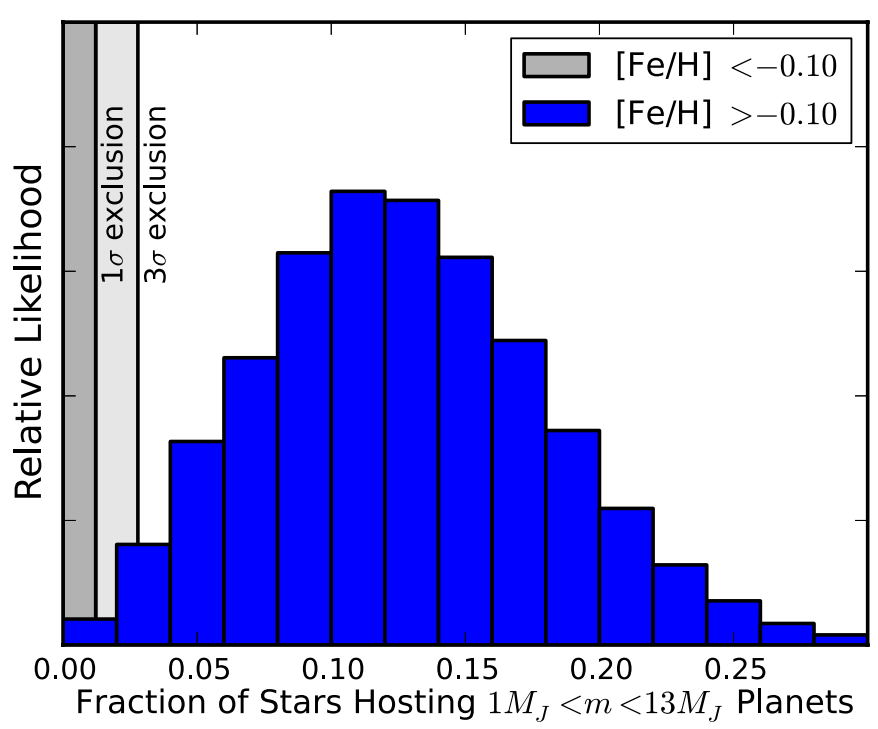

Figure 11. Planet occurrence for a high-metallicity subsample (blue) and $1 \sigma$ and $3 \sigma$ exclusion regions for a low-metallicity subsample (gray) of $\mathrm{M}$ dwarfs. In the low-metallicity subsample, we are able to rule out planet occurrence rates larger than $1.2 \%$ at $1 \sigma$ and $2.8 \%$ at $3 \sigma$, represented by the labeled vertical lines. The high-metallicity sample has a significantly higher occurrence rate than the low-metallicity sample, similar to the phenomenon observed for RV-detected planets at smaller separations.

(A color version of this figure is available in the online journal.)

spectral types previously (Johnson et al. 2010a); we follow the techniques of these authors but confine ourselves to strictly giant planets in the range $0<a<20 \mathrm{AU}$ orbiting stars of the M-dwarf spectral class.

We assume that stellar mass and metallicity produce separate effects on the giant planet occurrence rate, so that the fraction of stars with planets as a function of mass and metallicity can be written as a double power law,

$$
f(M, F)=C M^{a} 10^{b F},
$$

where $C$, $a$, and $b$ are constants, $M \equiv M / M \odot$, and $F \equiv[\mathrm{Fe} / \mathrm{H}]$.

In this analysis, we have a binary result: a star either has a giant planet, detectable as an RV acceleration or closed orbit, or it does not. Therefore, each of the $N$ stars in our sample represents a Bernoulli trial. Given $T$ total observed giant planets, if we assume the probability of a Doppler detection of a giant planet around any given star $i$ is $f\left(M_{i}, F_{i}\right)$, then by Bayes' theorem, the probability of a given model $X$ given our data $d$ is

$$
P(X \mid d) \propto P(X) \prod_{i}^{T} f\left(M_{i}, F_{i}\right) \times \prod_{j}^{N-T}\left[1-f\left(M_{j}, F_{j}\right)\right] .
$$

Our measurements of stellar masses and metallicities are imperfect. Therefore, we treat the masses and metallicities of these stars as probability distributions. We consider each star's mass and metallicity distribution to be a two-dimensional Gaussian with mean $M_{i}, F_{i}$ and standard deviation $\sigma_{M, i}, \sigma_{F, i}$ and call this term $p$. In this case, the predicted planet fraction for a star with mass $M_{i}$ and metallicity $F_{i}$ is

$$
f\left(M_{i}, F_{i}\right)=\iint p\left(M_{i}, F_{i}\right) f(M, F) d M d F .
$$

We can thus apply Equation (13) with varying parameters, $X=C, a, b$, to maximize $\mathcal{L}$ conditioned on the data. We elect 

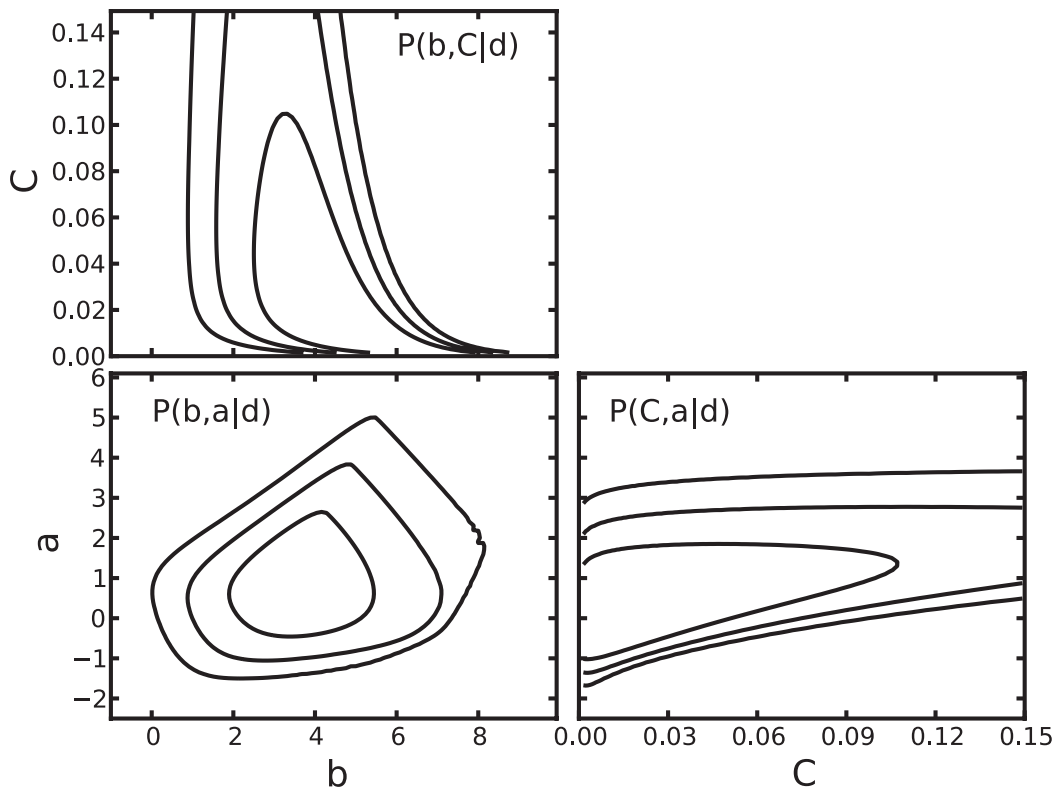

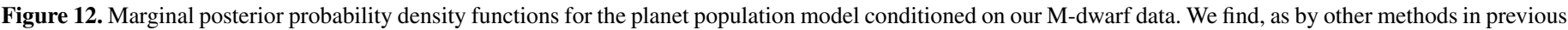
sections, that giant planet occurrence is a strong function of stellar metallicity, but may not depend strongly on stellar mass inside of the M spectral class.

to use uniform priors, instead of applying the results of previous studies as a prior. Johnson et al. (2010a) and Mortier et al. (2013) study a sample of stars including all stellar types $\mathrm{F}$ to $\mathrm{M}$, so their results may not represent our population well. More recent studies, such as Neves et al. (2013), are restricted to M dwarfs. However, while their techniques are similar, they only attempt to constrain metallicity, implicitly assuming $a=0$. Additionally on of the three detected planets in their sample is a planet smaller than Jupiter around a metal-poor star. As our sample is limited to planets larger than Jupiter, the resultant distribution found by these authors may not be representative of the population of giant planets $\left(m>1 M_{J}\right)$.

We find our giant planet fraction is described by the distribution function

$$
f(M, F)=0.039_{-0.028}^{+0.056} M^{0.8_{-0.9}^{+1.1}} 10^{(3.8 \pm 1.2) F} .
$$

The $1 \sigma$ confidence interval for $C$ is highly skewed, while the other two parameters are approximately normally distributed. In Figure 12, we plot the marginal posterior probability distribution functions for each pair of parameters. Perhaps not surprisingly, we find a covariance between $C$ and $b$. Because our metallicity parameter $b$ is so steep, small changes in $b$ must cause changes in $C$ to keep the giant planet fraction consistent at a given metallicity.

Our results are steeper in $b$ than Neves et al. (2013), although the giant planet occurrence rates at $[\mathrm{Fe} / \mathrm{H}] \sim 0.1$ are consistent between the two studies. This is likely due to the inclusion of a planet with a minimum mass of 0.7 Jupiter masses in the "Jovian" sample of these authors. This planet orbits a star with a metallicity $[\mathrm{Fe} / \mathrm{H}]=-0.19 \pm 0.08$, flattening the distribution with metallicity. The fact remains that, while the metallicity distribution of field stars is centered near $[\mathrm{Fe} / \mathrm{H}]=0.0$ with a standard deviation of $0.13 \mathrm{dex}$, there are presently no giant planets orbiting $M$ dwarfs with measured metallicities smaller than +0.08 in either the HARPS or HIRES sample. The giant planet distribution function must therefore be a strong function of stellar metallicity. Moreover, it is essential to develop improved methods to measure metallicities of low- mass stars, such as the techniques developed by Rojas-Ayala et al. (2012) and Mann et al. (2013).

\subsection{The Effect of Distant Binary Companions}

In the above analysis, we neglect binary stars where a test particle at $30 \mathrm{AU}$ would be in an unstable orbit, but include 14 binaries at wider separations. Although these systems formally allow stable orbits, Kaib et al. (2013) suggest these orbits can change significantly over time. Because the binary pair is weakly bound, interactions with the galactic tidal field or nearby passing stars can vary the binary orbit. The binary can then strongly perturb formerly stable planetary companions, potentially resulting in the ejection of planets from the system within $5 \mathrm{Gyr}$, our estimated age for the $\mathrm{M}$ dwarfs in our sample. None of our 10 wide binary systems show evidence for an RV acceleration, providing weak but tantalizing evidence in favor of this theory. If we repeat our analysis but neglect these stars as potential hosting systems, we find that $7.4 \% \pm 3.3 \%$ of single stars host giant planets, compared to $6.5 \% \pm 3.0 \%$ of our full sample. With zero detections in a sample of 14 wide binaries, we can only place an upper limit of $f_{\mathrm{pl}} \leqslant 0.20$ at $95 \%$ confidence on the occurrence rate of giant planets in wide binary systems. With more observations of stars with wide binary companions, the occurrence rate of planets orbiting true field stars can be compared to the rate for wide binaries.

\subsection{Sensitivity to Power-law Parameters}

The result for $f_{\mathrm{pl}}$ is dependent on the exact parameters of the planetary distribution function, as that function determines the number of missed (false negative) planets in our sample. To quantify the dependence of the planetary occurrence rate on our choice of $\alpha$ and $\beta$ we repeat our analysis over a grid of values for $\alpha$ and $\beta$. The giant planet occurrence rate as a function of these two parameters is shown in Figure 13. We find that there is only a weak relation between $\alpha$ and $f_{\mathrm{pl}}$ in the range $-2.0<\alpha<0.5$, where we might reasonably expect $\alpha$ to reside. $f_{\mathrm{pl}}$ depends more strongly on $\beta$, but our overall result does not change by more than $1 \sigma$ by selecting any $\beta$ in the range $-1.0<\beta<1.0$ for a 


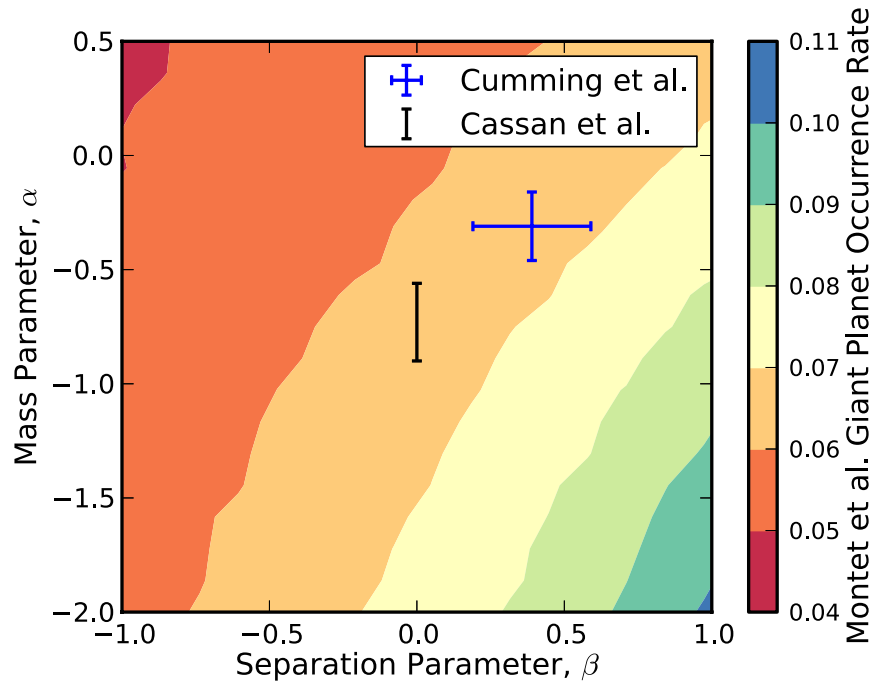

Figure 13. Calculated giant planet occurrence rate, $f_{\mathrm{pl}}$, as a function of the mass parameter index $\alpha$ and separation parameter index $\beta$. There is not a strong dependence on $\alpha$ or $\beta$; selecting $\alpha<-1.0$ and $\beta>0.5$ is required to affect our result at more than the $1 \sigma$ level. Labeled points include the Cumming et al. (2008) result for FGK stars, with $\alpha=-0.31 \pm 0.15$ and $\beta=0.39 \pm 0.15$, and the microlensing result of Cassan et al. (2012), who find $\alpha=-0.73 \pm 0.17$ and assume $\beta \equiv 0$.

(A color version of this figure is available in the online journal.)

given $\alpha$. Selecting any $\alpha$ or $\beta$ over this range affects our final result by less than a factor of two.

From our sample of targets alone, we are unable to place constraints on acceptable values of $\alpha$ and $\beta$. To constrain $\alpha$ and $\beta$, the occurrence rate of giant planets at a given mass or separation is required. We have determined the bulk occurrence rate of planets, but cannot uniquely determine their properties. With continued observations, as our RV accelerations "turn over" and become closed orbits, we will be able to determine the exact locations of giant planets around $\mathrm{M}$ dwarfs and constrain the power-law parameters. Alternatively, we can constrain $\alpha$ and $\beta$ by combining our results with those from microlensing observations.

\subsection{Comparison with Microlensing Results}

In Section 4.7, we showed that our bulk occurrence rate is not a strong function of $\alpha$ and $\beta$. However, the types and locations of our planets is a function of these parameters: if $\alpha$ is large, then most of our observed trends must be caused by large planets in wide orbits. Since microlensing results are most sensitive at projected separations corresponding to the Einstein radius, where $R_{E} \sim 3.5 \mathrm{AU}\left(M_{\star} / M_{\odot}\right)^{1 / 2}$, we can compare our results to microlensing planet occurrence studies. As our results will only be consistent with microlensing estimates of the planet occurrence rate at the Einstein radius for specific values of $\alpha$ and $\beta$, comparisons between the two methods will enable us to constrain $\alpha$ and $\beta$.

To compare the two sets of results, we assume the population of $M$ dwarfs observed by microlensing studies is similar to that targeted by RV surveys in the local neighborhood. We find evidence for a correlation between giant planet frequency and metallicity in our sample, similar to that found by previous RV analyses of planets with $a<2.5$ AU (Fischer \& Valenti 2005; Johnson \& Apps 2009). M dwarfs studied by microlensing are at distances larger than $1 \mathrm{kpc}$ and in the direction of the galactic bulge, along the galactic metallicity gradient (Rolleston et al. 2000). Measurements of the metal- licity of Cepheids suggest the iron content in the disk varies such that $d[\mathrm{Fe} / \mathrm{H}] / d r=-0.051 \pm 0.004 \mathrm{dex} \mathrm{kpc}^{-1}$ between 5 and $17 \mathrm{kpc}$ from the galactic center (Pedicelli et al. 2009). Thus, the microlensing $\mathrm{M}$ dwarfs may be more metal-rich than stars in the local neighborhood, so $f_{\mathrm{pl}}$ may be larger for the microlensing population than the RV population. Without spectra of galactic stellar planet-hosting lenses their true stellar properties are unknown. Programs dedicated to collecting spectra of galactic stellar planet-hosting lenses would greatly inform our knowledge of these stars and their planets.

If we assume the planet mass distribution function of Cumming et al. (2008), then from our analysis we would expect microlensing studies to measure a planet occurrence rate $f_{\mathrm{pl}}=0.056 \pm 0.023$ bound Jupiter-mass planets per star by analyzing signals from planets near the Einstein radius. Cassan et al. (2012) claim an occurrence rate of $10^{-0.62 \pm 0.22}\left(0.24_{-0.10}^{+0.16}\right)$ Saturn-mass planets at this separation. If we scale this occurrence rate to Jupiter-mass planets following the mass index observed in microlensing studies, $\alpha=-0.73 \pm 0.17$, then the observed microlensing density of Jupiter-mass planets would be $0.101 \pm 0.016$ planets per star, different from our expectation at $1.6 \sigma$. If (and only if) the two populations have intrinsically similar occurrence rates of giant planets, then the difference between the number of planets found must be due to a planet distribution different from the one used by Cumming et al. (2008). As the RV planet distribution was developed from an analysis of FGK stars, while the microlensing population generally consists of $\mathrm{M}$ dwarfs that may be preferentially metal-rich compared to stars in the local neighborhood, it may not be surprising if the RV planet population is intrinsically different from the microlensing planet population.

\subsubsection{Joint Constraints on $\alpha$}

We depart from our previously assumed values of $\alpha$ and $\beta$ to determine what values of $\alpha$ and $\beta$ satisfy both our observed RV accelerations and the results of Cassan et al. (2012). We assume the planet occurrence rate presented by Cassan et al. (2012) is representative of the planet population at the Einstein radius. Moreover, we assume planet orbital semimajor axes are distributed uniformly in logarithmic space following Öpik's law $(\beta=0)$, as microlensing studies assume. This is slightly shallower than what is observed in the RV planet population $(\beta=0.39 \pm 0.15)$, but since the RV population of giant planets likely underwent considerable migration this may be a reasonable assumption. We then vary $\alpha$, and for each value determine the space density of planets at $2.5 \mathrm{AU}$. We then compare our expected result to the result from Cassan et al. (2012), which we scale to Jupiter-mass planets according to our $\alpha$ parameter. We finally require $\alpha<0$ : despite the uncertainties in this mass parameter, previous studies agree that around M dwarfs, small planets are more common than massive planets (Swift et al. 2013; Morton \& Swift 2013).

We find microlensing results agree with our result for $f_{\mathrm{pl}}$ when $\alpha=-0.94 \pm 0.56$ (Figure 14). This result is consistent with the best-fitting values for $\alpha$ found by Gould et al. (2010) and Cassan et al. (2012). If we include the Cassan et al. (2012) result as a prior in our analysis, we find $\alpha=-0.77 \pm 0.22$. However, while our result agree with microlensing studies, our result for $\alpha$ is different from the Cumming et al. (2008) result for FGK stars at $1.1 \sigma$ and significantly different from the Bowler et al. (2010) constraints for A stars, which rule out all $\alpha<0.25$ with $90 \%$ confidence and all $\alpha<1.75$ with $50 \%$ confidence. Since microlensing predicts a larger number of planets found at the 


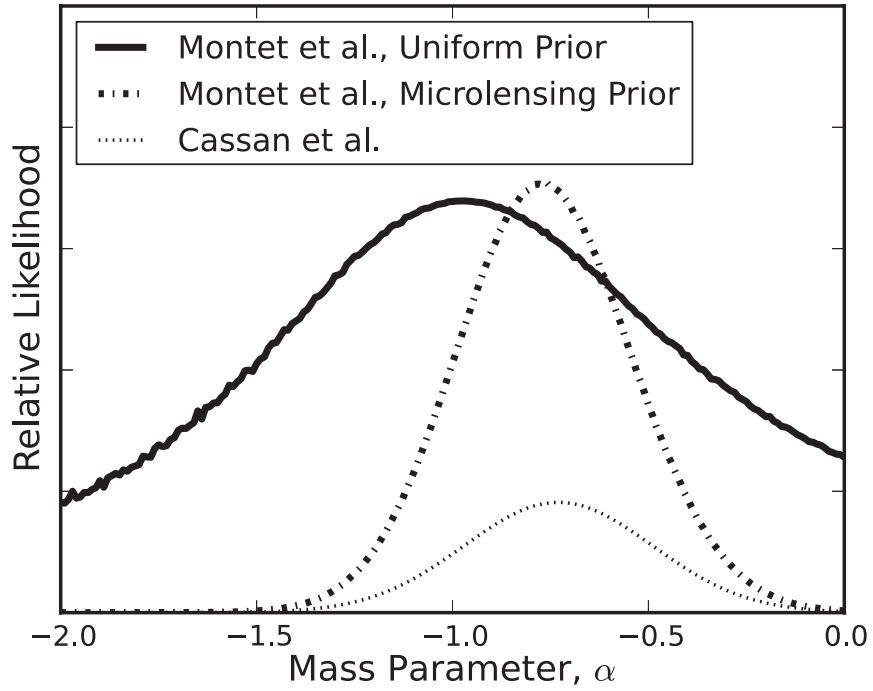

Figure 14. Relative likelihood values for the mass parameter $\alpha$, assuming the planets in our sample and microlensing systems are members of the same population. We find a maximum likelihood value of $\alpha=-0.94 \pm 0.56$, consistent with values of $\alpha$ found from analyses of microlensing planets but steeper than previous RV results for FGK stars at $1.1 \sigma$. This result may suggest the planet distribution function is different for $\mathrm{M}$ stars as compared to highermass stars. When we include the Cassan et al. (2012) result as a prior on our measurement, we find $\alpha=-0.77 \pm 0.22$.

Einstein radius relative to that expected by RV extrapolations, it is not surprising that we find a smaller value for $\alpha$ is required for our result to be consistent with the microlensing results: if the two populations are the same, there must be many lowmass giant planets below the simultaneous RV and imaging detectability limits than high-mass planets above the limits.

\subsubsection{Simultaneous Constraints on $\alpha$ and $\beta$}

We are not restricted to Öpik's law. We can allow both $\alpha$ and $\beta$ to vary, and compare the normalization of Cassan et al. (2012) for Saturn-mass objects at 2.5 AU to our projected planet density at that mass and separation (Figure 15). Performing this exercise, we find the most acceptable values of $\alpha$ and $\beta$ are correlated approximately along the line

$$
\alpha-\beta=-1
$$

That is, for every 1 dex increase in $\alpha, \beta$ must decrease by 1 dex to maintain a reasonable fit to both our result and the microlensing results.

\subsubsection{A Model-independent $f_{p l}$}

We can apply these relative likelihood values as priors to the occurrence rate as a function of $\alpha$ and $\beta$ shown in Figure 13 to determine an occurrence rate independent of our choices of $\alpha$ and $\beta$, but dependent on the RV and microlensing stars both being representative of similar populations. We assume our separation parameter must be in the range $-1.0<\beta<1.0$, consistent with the assumptions from previous microlensing studies, and allow our mass parameter to be any value subject to the constraints of Figure 15. By weighting our occurrence rates found in Section 4.7 in this manner, we find a most likely occurrence rate of $7.2 \% \pm 3.1 \%$, consistent with that found by assuming the power-law distribution of Cumming et al. (2008). As the measured planet frequency depends on the distribution function parameters, an improved value of the planet occurrence rate, either by this method, microlensing,

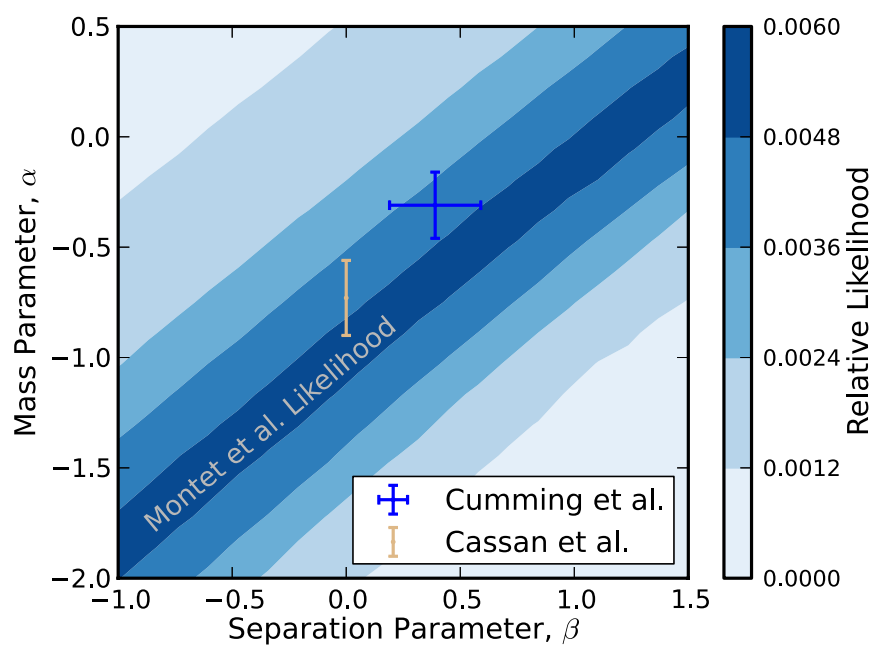

Figure 15. Relative likelihood values for the mass parameter $\alpha$, and separation parameter $\beta$. There is a maximum likelihood contour approximately along the line $\alpha-\beta=-1$, suggesting a relationship between the two parameters required to fit both our result and the microlensing results, assuming the local planets in our sample and microlensing systems are members of the same population. Points included in the plot are the Cumming et al. (2008) RV result (blue) and the Cassan et al. (2012) microlensing result (cyan), the latter of which assumes an Öpik's law value of $\beta=0$. The small discrepancy between our result and the Cumming et al. (2008) result may suggest the planet distribution function may differ between M dwarfs and FGK stars.

(A color version of this figure is available in the online journal.)

or through astrometry measured by Gaia (Casertano et al. 2008), will provide immediate constraints on the distribution function of giant planets. Similarly, improved constraints on the distribution parameters will enable an immediate improvement of the determination of the giant planet occurrence rate.

The Cumming et al. (2008) power-law parameters $\alpha$ and $\beta$ are less consistent with our results. This may suggest the planet distribution function around FGK stars is systematically different from the planet distribution function around $\mathrm{M}$ dwarfs. As Bowler et al. (2010) find an even larger value for $\alpha$ in their study of retired A stars (excluding all $\alpha<0$ ), which matches comparison studies between RV surveys and high-contrast imaging searches (Crepp \& Johnson 2011), this possibility is certainly plausible. With additional $M$ dwarfs targeted by a combination of RV observations with longer time baselines and high-contrast imaging to improve the estimate of the occurrence rate, we will be able to directly probe this possibility.

\section{SUMMARY AND CONCLUSION}

We have analyzed a collection of 111 nearby $M$ dwarfs observed in RV surveys with a median time baseline of $11.8 \mathrm{yr}$ in a search for long-term RV accelerations. We have developed a new technique to determine the incidence of giant planets in which we target systems with such accelerations using AO imaging to "peer beyond the horizon" set by Doppler time baselines. With a relatively short exposure image using the Keck AO system, we can eliminate the possibility of binary stellar companions and massive brown dwarfs. We conclude with high statistical confidence that accelerations without a directly imaged companion are likely caused by a planet in a wide orbit.

Accounting for false positive and false negative rates, we find that $6.5 \% \pm 3.0 \%$ of $\mathrm{M}$ dwarfs host a giant planet with mass $1<m / M_{J}<13$ and semimajor axis $0<a<20 \mathrm{AU}$, assuming such planets are distributed following the power-law parameters estimated by Cumming et al. (2008). The exact 
integrated planet occurrence rate does not depend strongly on the distribution function parameters chosen. We find evidence for a correlation between giant planet frequency and stellar metallicity, similar to that observed in the RV-detected planet population. Additional follow-up work confirming this result would suggest giant planets in wide orbits may form in the same way as the RV-detected giant planets. Observations of more stars are needed to determine if a correlation exists between planet occurrence at wide separations and stellar mass inside of the M-dwarf spectral class.

Our overall occurrence rate is consistent with what might be expected based on the results of microlensing planet search surveys. However, if the giant planet distribution is given as a double power law similar to that found by Cumming et al. (2008), such that $d^{2} N \propto M^{\alpha} a^{\beta} d \ln M d \ln a$, with $\alpha=$ $-0.31 \pm 0.20$ and $\beta=0.39 \pm 0.15$, where $\alpha$ and $\beta$ are planet distribution power-law indices defined in Equation (7), then microlensing studies overestimate the giant planet occurrence rate. From our bulk occurrence rate, we determine an expected planet detection rate for microlensing studies which depends on our chosen planet distribution function. By assuming an Öpik's law distribution (i.e., flat in $\log a$ ), the microlensing planet occurrence rate is consistent with our result if the planet population is represented by the power-law $d N \propto$ $m^{-0.94 \pm 0.56} d \log m$. This value for $\alpha$ is consistent with previous M-dwarf studies conducted by microlensing planet search teams (Gould et al. 2010; Cassan et al. 2012). We also find other nonÖpik distributions can be chosen to simultaneously explain our results and the microlensing results; these fall approximately on the line $\alpha-\beta=-1$. Moreover, an improved estimate of the giant planet occurrence rate, as measured by Gaia, can be combined with our results to provide enhanced constraints on $\alpha$ and $\beta$.

Our knowledge of planets around $\mathrm{M}$ dwarfs has significantly improved in the last few years thanks to both targeted RV searches and high-contrast imaging campaigns (Apps et al. 2010; Bowler et al. 2012). As such surveys continue, they will begin to confirm and characterize planets in wider orbits, pushing into the domain currently only studied by microlensing studies. To directly compare these populations, understanding the properties of host stars to planets found by microlensing will be extremely important; when possible, every effort should be made to collect spectroscopic follow-up data on microlensing events to determine the physical properties of lens host stars to better understand both the planet population around $\mathrm{M}$ dwarfs and how it changes across the galaxy.

The method developed in this paper can be extended to highermass stars with little difficulty. For example, a large sample of $\mathrm{K}$ dwarfs has been observed by the CPS collaboration. This sample is larger, has more observations, and exhibits less astrophysical jitter than our M-dwarf sample; all of these factors improve our ability to detect RV accelerations. However, the stars are more luminous and on average more distant, complicating AO searches. Care must be taken to ensure lowmass stellar companions are accounted for, as AO imaging may not be sensitive to all $\mathrm{M}$-dwarf companions to $\mathrm{K}$ dwarfs without longer observations or the use of ADI. In the future, we intend to apply this technique to the CPS K dwarfs to determine the planet occurrence rate around higher-mass stars and compare to the $\mathrm{M}$ dwarfs.

Most of the data presented herein were obtained at the W.M. Keck Observatory, which is operated as a scientific partnership among the California Institute of Technology, the University of California, and the National Aeronautics and Space Administration. The Observatory was made possible by the generous financial support of the W.M. Keck Foundation. We thank the staff of the Palomar Observatory for their help in maximizing the efficiency and quality of the 200" Hale Telescope. This publication makes use of data products from the Two Micron All-Sky Survey, which is a joint project of the University of Massachusetts and the Infrared Processing and Analysis Center/California Institute of Technology, funded by the National Aeronautics and Space Administration and the National Science Foundation. We made use of the SIMBAD database operated at CDS, Strasbourg, France, and NASA's Astrophysics Data System Bibliographic Services. We thank Jon Swift for collecting NIRC2 observations of HIP 57050. We also thank Brendan Bowler for helpful comments on an early version of this manuscript. B.T.M. is supported by the National Science Foundation Graduate Research Fellowship under grant No. DGE1144469. The TRENDS high-contrast imaging program is supported by NASA Origins grant NNX13AB03G. J.A.J. is supported by generous grants from the David and Lucile Packard Foundation and the Alfred P. Sloan Foundation. B.T.M. would also like to thank the Statistical and Applied Mathematical Sciences Institute; conversations at the 2013 June Modern Statistical and Computational Methods for Analysis of Kepler Data workshop at SAMSI improved the final version of this manuscript.

Finally, the authors recognize and acknowledge the very significant cultural role and reverence that the summit of Mauna Kea has always had within the indigenous Hawaiian community. We are most fortunate to have the opportunity to conduct observations from this mountain.

\section{APPENDIX A NOTES ON INDIVIDUAL TARGETS}

\section{A.1. $G l 849$}

The RV data for G1 849 exhibits a clear planetary signal from the known companion G1 849b. The residuals to the best-fitting orbit for this planet exhibit strong curvature, motivating our twoplanet fit. Moreover, there is no correlation between this long period signal and stellar magnetic activity, suggesting the planet is not the result of an apparent velocity change during the star's magnetic cycle. To determine the orbital parameters of both planets, we utilize emcee, an affine invariant MCMC ensemble sampler (Foreman-Mackey et al. 2013). For both planets, we fit five orbital parameters: the eccentricity $e$, argument of periapsis $\omega$, time at which a transit would occur $t_{\varpi=90}$, Doppler semiamplitude $K$ (or the product of the planet mass and the inclination $m \sin i$ ), and planet orbital period $P$. We also include the systemic RV $\gamma$ as a free parameter, as well as a velocity offset between observations taken before 2004 August 18 and after that date, corresponding to an upgrade of the HIRES CCD detector (Wright et al. 2011).

Due to the curvature in the outer planet's orbit, we are able to constrain the mass and period of both companions. As shown in Figure 16, the orbit of the outer planet is only weakly constrained. Nevertheless, the data can rule out orbits with $m \sin i>2.0 M_{J}$. Moreover, we refine the inner planet's parameters: we find the "b" component's best-fitting mass and period increase slightly, but the distributions for each are consistent with those found by Butler et al. (2006). Our parameters for each planet are included in Table 5. 

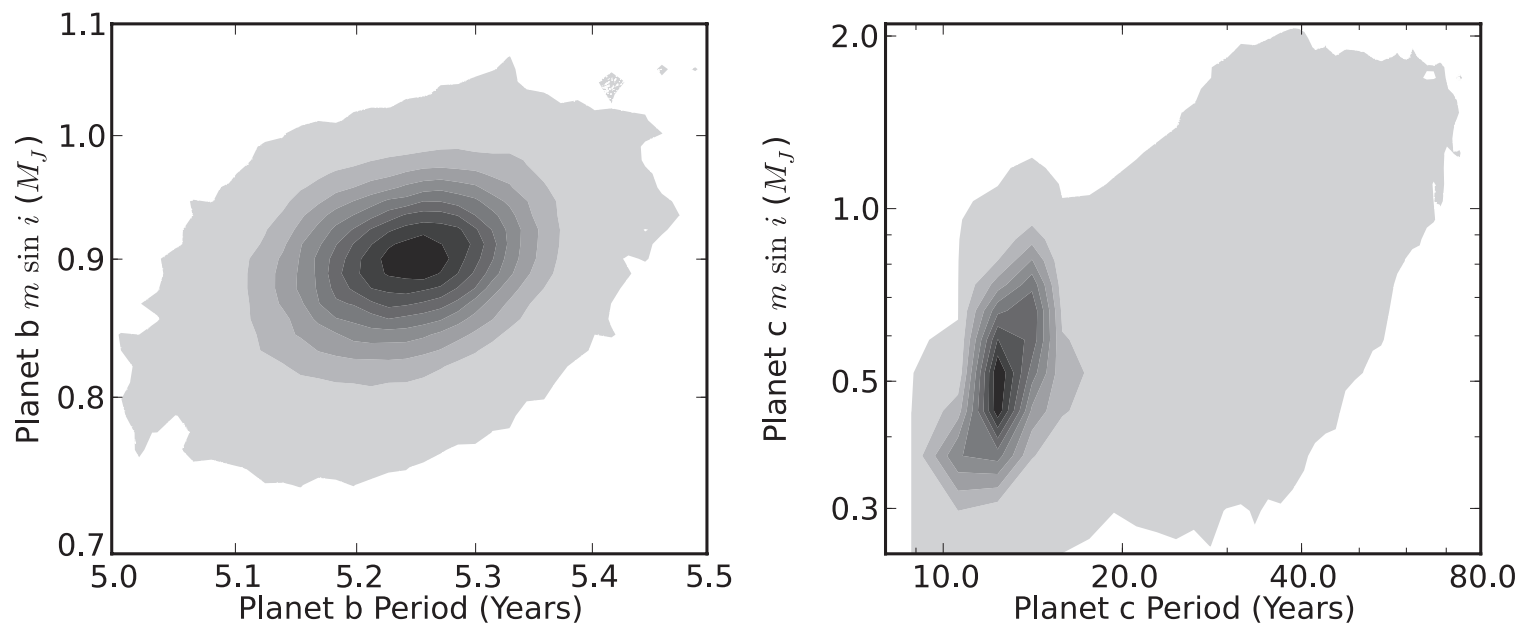

Figure 16. Position of (left) Gl 849b and (right) Gl 849c in the mass-period plane. The orbital parameters for the inner planet are much more tightly constrained than the outer planet. Depending on the exact shape of the planet distribution function, the inner planet may have more than a 50\% probability of being more massive than Jupiter when orientation uncertainties are taken into account.

Table 5

Orbital Parameters for G1 849

\begin{tabular}{lcccc}
\hline \hline Parameter & Mean & $50 \%$ & $15.8 \%^{\mathrm{a}}$ & $84.2 \%^{\mathrm{a}}$ \\
\hline Planet b & & & & \\
Orbital period $P(\mathrm{yr})$ & 5.241 & 5.243 & -0.067 & +0.064 \\
Planet mass ${ }^{\mathrm{b}} \sin i\left(M_{J}\right)$ & 0.899 & 0.900 & -0.045 & +0.043 \\
Time of potential transit $t_{\varpi=90}(\mathrm{JD}-2,440,000)$ & 537.3 & 536.9 & -161.3 & +164.7 \\
$e^{1 / 2} \cos \omega$ & -0.048 & -0.059 & -0.105 & +0.122 \\
$e^{1 / 2} \sin \omega$ & 0.099 & 0.116 & -0.161 & +0.114 \\
Planet c & & & & \\
Orbital period $P(\mathrm{yr})$ & 24.04 & 19.35 & -5.93 & +17.20 \\
Planet mass ${ }^{\mathrm{b}} \sin i\left(M_{J}\right)$ & 0.773 & 0.702 & -0.203 & +0.344 \\
Time of potential transit $t_{\varpi=90}(\mathrm{JD}-2,440,000)$ & 3586.3 & 5660.3 & -7356.0 & +2387.6 \\
$e^{1 / 2} \cos \omega$ & -0.311 & -0.346 & -0.185 & +0.260 \\
$e^{1 / 2} \sin \omega$ & -0.348 & -0.361 & -0.234 & +0.253 \\
System parameters & & & & \\
HIRES detector upgrade offset $\left(\mathrm{m} \mathrm{s}^{-1}\right)$ & 17.07 & 17.18 & -5.25 & +5.01 \\
\hline
\end{tabular}

\section{Notes.}

${ }^{\text {a }}$ Values given relative to the $50 \%$ data point.

${ }^{\mathrm{b}}$ Assuming a stellar mass of $0.49 M_{\odot}$.

\section{A.2. HIP 109555}

When observing HIP 109555 we detected a possible faint companion object located tens of arcseconds away. To prove this companion is not associated with the primary but is instead unrelated, we compare the proper motion of both objects by identifying them in the 2MASS catalog (Skrutskie et al. 2006) and the Palomar Observatory Sky Survey (POSS; Abell 1959). Comparing the POSS data collected 1950 July 16 to the 2MASS observation, we detect a proper motion for HIP 109555 of 0.36 arcsec $\mathrm{yr}^{-1}$, consistent with previously published results (van Leeuwen 2007). The hypothetical companion motion,

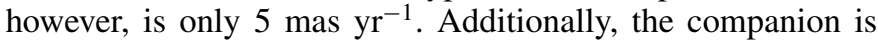
bluer in colors derived using the 2MASS $J, H$, and $K$ filters than HIP 109555. These are both consistent with the companion being a distant background object, and we neglect its presence in our analysis.

\section{A.3. HIP 57050}

We observed HIP 57050 (=GJ1148) on 2012 December 27 using the $K_{s}$ filter on NIRC2. Our imaging is only complete at separations smaller than 1 arcsec, corresponding to a projected

separation of $11 \mathrm{AU}$. This does not enable us to rule out most stellar companions that could cause our observed RV trends, as shown in Figure 17. If the observed trend is caused by a stellarmass companion, the companion is likely beyond $10 \mathrm{AU}$, which corresponds to a separation of 0.9 arcsec. Thus any stellar companions at their maximum separation that could cause this trend would be expected to be found in a seeing-limited survey. We find no evidence for such a companion. While unlikely, additional AO observations with a wider field of view are required to fully eliminate the possibility that a low-mass star exists.

\section{A.4. HIP 63510}

HIP 63510B (Ross 458) is an M7 brown dwarf orbiting an M0.5 dwarf at approximately 3 AU (Beuzit et al. 2004). Twelve years of RV observations suggest an orbit with a period of $13.9 \mathrm{yr}$, an eccentricity of 0.32 , and a minimum mass $m \sin i=67.9 M_{J}$, suggesting a nearly edge-on orbit. We estimate a detection efficiency of 1.000 in an RV survey, which is not surprising considering the stellar RV semiamplitude is $K=1.24 \mathrm{~km} \mathrm{~s}^{-1}$. This system contains a second companion which is separated from the host star by $1100 \mathrm{AU}$ (Goldman et al. 2010; Scholz 2010). 


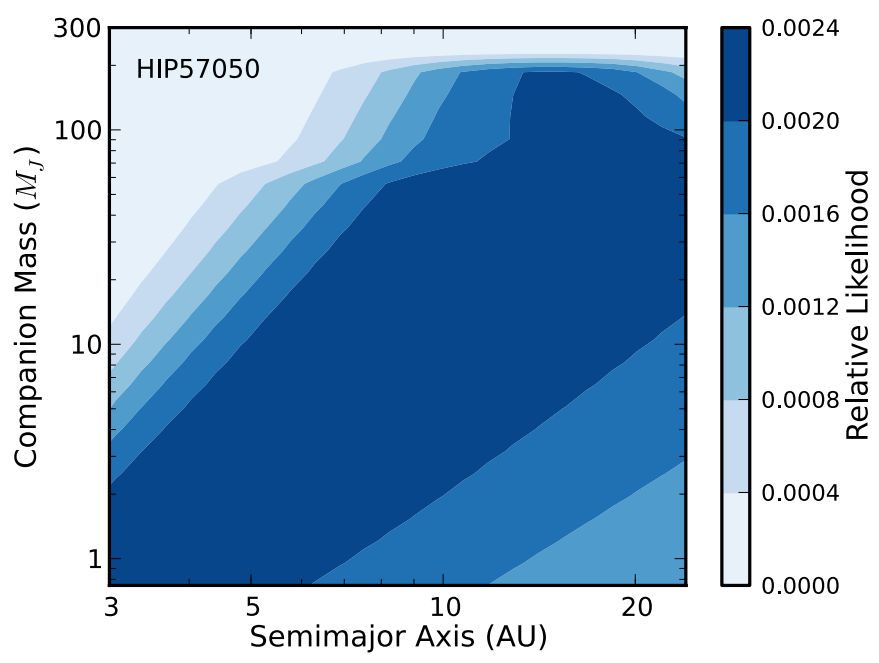

Figure 17. Probability contours displaying the location of a giant companion orbiting HIP 57050, given that exactly one such planet exists, when the RV data is combined with adaptive optics imaging and 2MASS data. Because the AO imagery only extends to $11 \mathrm{AU}$, there is a small region of parameter space where a low-mass M-dwarf companion could reside. Additional AO observations with a wider field of view would be required to rule out this possibility. Lower-mass companions are allowed in shorter orbital periods due to possible curvature in the radial velocity data.

(A color version of this figure is available in the online journal.)

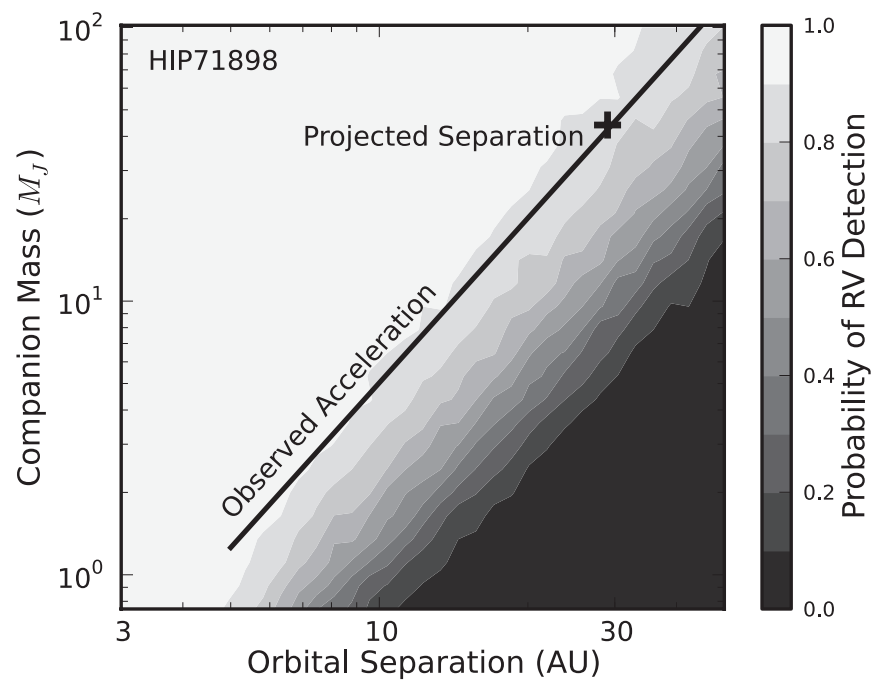

Figure 18. Probability contours displaying the likelihood that a planet of a given mass and semimajor axis would be detected around HIP 71898 in the CPS RV survey. The diagonal line represents companions that would produce an acceleration of $8.6 \pm 0.4 \mathrm{~m} \mathrm{~s}^{-1} \mathrm{yr}^{-1}$ in an edge on system when the companion was moving along the observer's line of sight. The + marks the spot at which a $45 M_{J}$ companion at $30 \mathrm{AU}$ would reside.

\section{A.5. HIP 71898}

HIP 71898B is an L0 dwarf in a wide orbit around an M3.5 dwarf. Golimowski et al. (2004) report a projected separation of $30.01 \pm 3.78 \mathrm{AU}$. This target has an RV baseline of $14 \mathrm{yr}$, over which 30 observations were collected. From these observations we measure an acceleration of $8.6 \pm 0.4 \mathrm{~m} \mathrm{~s}^{-1} \mathrm{yr}^{-1}$. At $30 \mathrm{AU}$, this would suggest a minimum dynamical mass $m \sin i>$ $45 M_{J}$, consistent with an L0 dwarf. A detectability plot for companions to HIP 71898 is shown in Figure 18. The observed acceleration lies near a contour representing a 0.9 probability of $\mathrm{RV}$ detection, so it is not surprising this companion was detected by CPS.

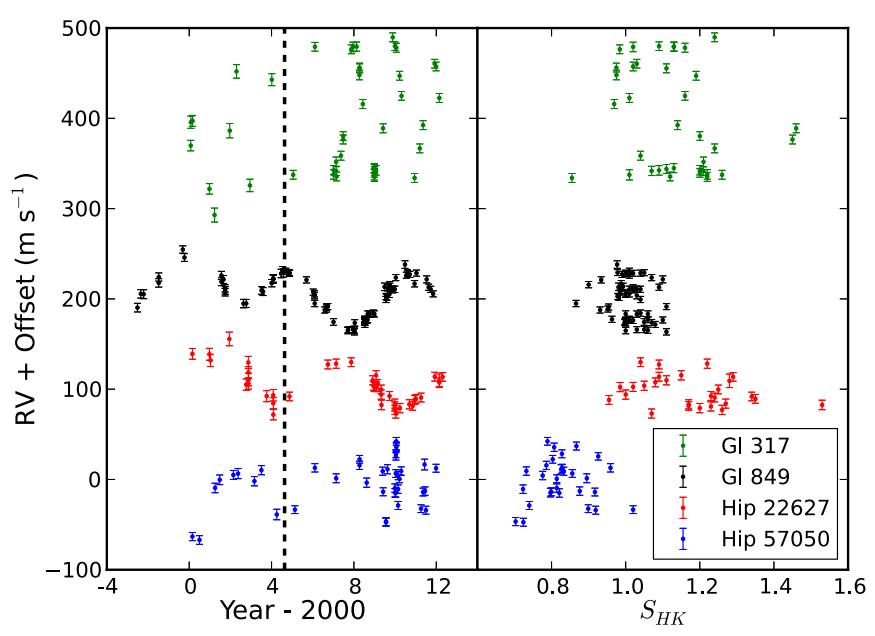

Figure 19. Left: RV time series for our four systems exhibiting long-term RV accelerations. The vertical line in 2004 represents the HIRES detector upgrade in August of that year. Right: RVs as a function of $S_{\mathrm{HK}}$. All four RV accelerations are visible, but none of the RV data appear to correlate with $S_{\mathrm{HK}}$, commonly used as a proxy for stellar chromospheric activity.

(A color version of this figure is available in the online journal.)

\section{A.6. Gl569}

Gl 569B is a brown dwarf binary, with an M8.5+M9 pair orbiting each other every $870 \pm 9$ days. The system has a combined mass of $0.140_{-0.008}^{+0.009} M_{\odot}$ (Dupuy et al. 2010) and is separated from the primary, an M3.5 dwarf, by a projected separation of 5 arcsec, or 47 AU (Femenía et al. 2011). The maximum RV acceleration from such a companion is $3.7 \mathrm{~m} \mathrm{~s}^{-1} \mathrm{yr}^{-1}$. For this star, we have a $5.1 \mathrm{yr}$ baseline and the median $\sigma$ is $15 \mathrm{~m} \mathrm{~s}^{-1}$. By injecting simulated companions, we estimate an RV detection efficiency near zero for these companions. Thus it is not surprising that it is missed in our sample.

\section{A.7. Gl $229 B$}

G1 229B (HD 42581) is a T7 dwarf at a projected separation of 44 AU (Faherty et al. 2009). This companion has been directly imaged (Nakajima et al. 1995) but not detected as a strong acceleration through RV variations. As with Gl 569, this object is beyond our range for efficient brown dwarf detection through RV observations. If we assume a mass of $40 M_{J}$, we would expect a maximal RV acceleration of $1.1 \mathrm{~m} \mathrm{~s}^{-1} \mathrm{yr}^{-1}$. Thus, again we should not be surprised it is not detected.

\section{APPENDIX B}

\section{A BRIEF NOTE ON RADIAL VELOCITIES AND MAGNETIC ACTIVITY}

We account for the possibility that any apparent RV accelerations may be induced by magnetic activity statistically, as described in Section 3.2. Often, the $S_{\mathrm{HK}}$ value, a measure of the ratio of flux in the $\mathrm{Ca}$ II line cores to flux in nearby continuum regions, is taken as a proxy for chromospheric activity (Wilson 1968; Henry et al. 1996). While not a perfect measure, it is comforting to note that the observed RVs do not correlate with $S_{\mathrm{HK}}$ in any of our stars with long-term RV accelerations. The RVs for our systems with detected accelerations as well as $S_{\mathrm{HK}}$ for observations after the HIRES detector upgrade are included in Figure 19 and Table 6. 
Table 6

RVs and $S_{\mathrm{HK}}$ Values for Systems with Long-term RV Accelerations

\begin{tabular}{|c|c|c|c|c|c|c|c|}
\hline $\mathrm{JD}-2,440,000$ & $\begin{array}{c}\mathrm{RV} \\
\left(\mathrm{m} \mathrm{s}^{-1}\right)\end{array}$ & $\begin{array}{c}\sigma_{\mathrm{RV}} \\
\left(\mathrm{m} \mathrm{s}^{-1}\right)\end{array}$ & $S_{\mathrm{HK}}$ & JD-244,000 & $\begin{array}{c}\mathrm{RV} \\
\left(\mathrm{m} \mathrm{s}^{-1}\right)\end{array}$ & $\begin{array}{c}\sigma_{\mathrm{RV}} \\
\left(\mathrm{m} \mathrm{s}^{-1}\right)\end{array}$ & $S_{\mathrm{HK}}$ \\
\hline \multicolumn{8}{|l|}{ Gl 317} \\
\hline 11550.993 & 369.80 & 5.83 & $\mathrm{~N} / \mathrm{A}$ & 14544.905 & 456.22 & 4.92 & 0.97 \\
\hline 11552.990 & 395.68 & 6.74 & $\mathrm{~N} / \mathrm{A}$ & 14545.894 & 455.37 & 4.96 & 1.11 \\
\hline 11582.891 & 397.16 & 6.08 & N/A & 14603.777 & 415.82 & 5.00 & 0.97 \\
\hline 11883.101 & 321.88 & 5.83 & N/A & 14806.029 & 344.83 & 5.02 & 1.13 \\
\hline 11973.795 & 292.84 & 7.74 & $\mathrm{~N} / \mathrm{A}$ & 14807.069 & 337.39 & 5.70 & 1.01 \\
\hline 12243.073 & 386.34 & 7.95 & $\mathrm{~N} / \mathrm{A}$ & 14808.138 & 343.93 & 4.94 & 1.11 \\
\hline 12362.949 & 451.96 & 7.50 & $\mathrm{~N} / \mathrm{A}$ & 14809.059 & 335.15 & 4.95 & 1.22 \\
\hline 12601.045 & 325.69 & 6.93 & $\mathrm{~N} / \mathrm{A}$ & 14810.161 & 339.65 & 4.87 & 1.20 \\
\hline 12989.125 & 442.81 & 6.64 & $\mathrm{~N} / \mathrm{A}$ & 14811.128 & 341.51 & 4.93 & 1.21 \\
\hline 13369.016 & 337.48 & 4.90 & 1.26 & 14839.107 & 342.36 & 5.26 & 1.09 \\
\hline 13753.983 & 479.22 & 4.85 & 1.13 & 14963.795 & 388.95 & 4.98 & 1.46 \\
\hline 14084.001 & 337.88 & 5.29 & 1.22 & 15134.090 & 489.75 & 4.90 & 1.24 \\
\hline 14086.141 & 342.52 & 5.21 & 1.20 & 15173.079 & 479.92 & 4.79 & 1.09 \\
\hline 14130.082 & 351.80 & 5.37 & 1.21 & 15199.017 & 478.18 & 4.98 & 1.16 \\
\hline 14131.014 & 341.73 & 5.11 & 1.07 & 15255.869 & 447.15 & 4.89 & 1.19 \\
\hline 14138.932 & 335.57 & 4.86 & 1.12 & 15289.857 & 424.86 & 4.82 & 1.16 \\
\hline 14216.733 & 358.57 & 4.95 & 1.04 & 15522.057 & 333.93 & 4.97 & 0.85 \\
\hline 14255.743 & 376.54 & 4.92 & 1.45 & 15613.960 & 366.70 & 4.92 & 1.24 \\
\hline 14255.749 & 380.38 & 4.79 & 1.20 & 15672.848 & 392.45 & 4.92 & 1.14 \\
\hline 14400.110 & 476.46 & 4.91 & 0.98 & 15878.127 & 460.49 & 4.81 & 1.03 \\
\hline 14428.062 & 479.05 & 5.29 & 1.02 & 15903.017 & 457.41 & 4.79 & 1.02 \\
\hline 14492.901 & 479.46 & 5.05 & 1.13 & 15960.986 & 422.57 & 4.98 & 1.01 \\
\hline 14543.948 & 448.01 & 5.34 & 0.97 & & & & \\
\hline \multicolumn{8}{|l|}{ G1 849} \\
\hline 10606.068 & 190.31 & 4.78 & $\mathrm{~N} / \mathrm{A}$ & 14455.744 & 165.29 & 3.45 & 1.06 \\
\hline 10666.001 & 205.60 & 4.69 & $\mathrm{~N} / \mathrm{A}$ & 14456.733 & 163.51 & 3.48 & 1.11 \\
\hline 10715.957 & 205.19 & 4.99 & $\mathrm{~N} / \mathrm{A}$ & 14460.742 & 173.41 & 3.53 & 1.00 \\
\hline 10983.038 & 217.69 & 4.67 & $\mathrm{~N} / \mathrm{A}$ & 14634.083 & 176.64 & 3.34 & 1.10 \\
\hline 10984.084 & 224.23 & 4.55 & $\mathrm{~N} / \mathrm{A}$ & 14635.042 & 173.89 & 3.32 & 1.00 \\
\hline 11410.021 & 254.67 & 4.08 & $\mathrm{~N} / \mathrm{A}$ & 14636.051 & 176.71 & 3.33 & 1.01 \\
\hline 11439.865 & 245.85 & 4.30 & $\mathrm{~N} / \mathrm{A}$ & 14637.116 & 176.23 & 3.31 & 1.00 \\
\hline 12095.081 & 225.97 & 4.52 & $\mathrm{~N} / \mathrm{A}$ & 14638.059 & 177.42 & 3.41 & 0.96 \\
\hline 12096.046 & 219.06 & 4.38 & N/A & 14639.067 & 174.78 & 3.42 & 1.00 \\
\hline 12133.013 & 221.49 & 4.39 & $\mathrm{~N} / \mathrm{A}$ & 14640.115 & 171.70 & 3.36 & 1.08 \\
\hline 12160.909 & 211.60 & 4.10 & N/A & 14641.117 & 173.84 & 3.38 & 1.07 \\
\hline 12161.846 & 207.39 & 4.19 & N/A & 14644.113 & 177.39 & 3.40 & 1.01 \\
\hline 12162.887 & 209.34 & 4.22 & $\mathrm{~N} / \mathrm{A}$ & 14674.936 & 176.17 & 3.40 & 1.02 \\
\hline 12486.968 & 194.80 & 4.66 & $\mathrm{~N} / \mathrm{A}$ & 14688.952 & 177.11 & 3.40 & 1.06 \\
\hline 12535.852 & 194.96 & 4.43 & $\mathrm{~N} / \mathrm{A}$ & 14690.005 & 183.22 & 3.51 & 1.06 \\
\hline 12807.011 & 209.44 & 4.30 & $\mathrm{~N} / \mathrm{A}$ & 14721.949 & 183.11 & 3.52 & 1.03 \\
\hline 12834.013 & 208.07 & 4.39 & $\mathrm{~N} / \mathrm{A}$ & 14790.752 & 184.27 & 3.43 & 1.04 \\
\hline 12989.720 & 217.41 & 4.08 & $\mathrm{~N} / \mathrm{A}$ & 14807.793 & 183.33 & 3.47 & 1.00 \\
\hline 13014.710 & 222.75 & 4.27 & $\mathrm{~N} / \mathrm{A}$ & 14989.063 & 213.37 & 4.17 & 0.98 \\
\hline 13015.711 & 221.97 & 4.60 & $\mathrm{~N} / \mathrm{A}$ & 15015.047 & 199.35 & 3.42 & 1.04 \\
\hline 13016.706 & 222.33 & 4.07 & N/A & 15016.074 & 202.71 & 3.36 & 0.98 \\
\hline 13154.080 & 228.16 & 4.76 & $\mathrm{~N} / \mathrm{A}$ & 15029.019 & 201.72 & 3.52 & 0.98 \\
\hline 13180.108 & 231.43 & 4.45 & N/A & 15043.042 & 212.32 & 3.40 & 1.02 \\
\hline 13196.931 & 228.82 & 4.63 & N/A & 15048.996 & 209.45 & 3.39 & 0.98 \\
\hline 13238.929 & 230.55 & 3.44 & 1.01 & 15075.082 & 205.14 & 3.55 & 1.00 \\
\hline 13301.838 & 228.44 & 3.39 & 1.00 & 15080.084 & 215.78 & 3.50 & 0.90 \\
\hline 13302.742 & 228.98 & 3.32 & 1.05 & 15082.073 & 213.97 & 3.44 & 0.99 \\
\hline 13303.798 & 228.40 & 3.27 & 1.02 & 15134.922 & 210.04 & 3.41 & 1.02 \\
\hline 13603.939 & 221.04 & 3.43 & 0.93 & 15135.876 & 210.90 & 3.37 & 1.03 \\
\hline 13724.712 & 207.52 & 3.39 & 0.98 & 15169.797 & 210.64 & 3.55 & 1.01 \\
\hline 13746.715 & 205.70 & 3.60 & 1.01 & 15188.725 & 223.58 & 3.42 & 1.07 \\
\hline 13746.721 & 203.74 & 3.72 & 1.03 & 15352.082 & 238.03 & 4.18 & 0.98 \\
\hline 13749.698 & 194.88 & 3.51 & 0.87 & 15376.032 & 226.26 & 3.36 & 1.01 \\
\hline 13927.015 & 187.71 & 3.42 & 0.93 & 15395.958 & 229.16 & 3.32 & 0.98 \\
\hline 13959.087 & 191.03 & 3.34 & 1.90 & 15397.048 & 227.85 & 3.36 & 1.00 \\
\hline 13960.955 & 188.72 & 3.31 & 0.95 & 15436.111 & 227.10 & 3.40 & 0.99 \\
\hline 13960.962 & 191.05 & 3.32 & 0.95 & 15521.801 & 216.77 & 3.53 & 0.99 \\
\hline 13983.000 & 191.46 & 3.36 & 1.11 & 15555.792 & 228.55 & 3.38 & 1.04 \\
\hline 14083.750 & 174.45 & 3.67 & 1.05 & 15736.122 & 221.64 & 3.86 & 1.10 \\
\hline 14337.074 & 164.82 & 3.45 & 1.00 & 15770.878 & 212.94 & 3.41 & 1.09 \\
\hline
\end{tabular}


Table 6

(Continued)

\begin{tabular}{|c|c|c|c|c|c|c|c|}
\hline $\mathrm{JD}-2,440,000$ & $\begin{array}{c}\mathrm{RV} \\
\left(\mathrm{m} \mathrm{s}^{-1}\right)\end{array}$ & $\begin{array}{c}\sigma_{\mathrm{RV}} \\
\left(\mathrm{m} \mathrm{s}^{-1}\right)\end{array}$ & $S_{\mathrm{HK}}$ & $\mathrm{JD}-244,000$ & $\begin{array}{c}\mathrm{RV} \\
\left(\mathrm{m} \mathrm{s}^{-1}\right)\end{array}$ & $\begin{array}{c}\sigma_{\mathrm{RV}} \\
\left(\mathrm{m} \mathrm{s}^{-1}\right)\end{array}$ & $S_{\mathrm{HK}}$ \\
\hline 14343.872 & 165.90 & 3.35 & 1.03 & 15807.063 & 210.62 & 3.40 & 1.04 \\
\hline 14429.742 & 166.12 & 3.44 & 1.05 & 15851.759 & 205.57 & 3.33 & 1.00 \\
\hline \multicolumn{8}{|l|}{ Hip 22627} \\
\hline 11580.831 & 139.11 & 6.00 & $\mathrm{~N} / \mathrm{A}$ & 14838.995 & 115.36 & 5.10 & 1.15 \\
\hline 11882.888 & 138.64 & 6.58 & $\mathrm{~N} / \mathrm{A}$ & 14846.957 & 102.80 & 5.28 & 2.81 \\
\hline 11901.002 & 131.80 & 6.77 & $\mathrm{~N} / \mathrm{A}$ & 14864.957 & 105.69 & 5.05 & 1.97 \\
\hline 12235.849 & 155.64 & 7.57 & $\mathrm{~N} / \mathrm{A}$ & 14928.732 & 99.68 & 4.86 & 1.25 \\
\hline 12536.088 & 105.11 & 6.31 & $\mathrm{~N} / \mathrm{A}$ & 14929.726 & 94.02 & 5.14 & 1.00 \\
\hline 12572.991 & 129.52 & 6.78 & $\mathrm{~N} / \mathrm{A}$ & 14934.731 & 82.53 & 5.28 & 1.53 \\
\hline 12573.950 & 118.58 & 6.32 & $\mathrm{~N} / \mathrm{A}$ & 15077.110 & 92.45 & 4.91 & 1.23 \\
\hline 12575.047 & 106.25 & 6.29 & $\mathrm{~N} / \mathrm{A}$ & 15170.784 & 80.88 & 5.08 & 1.23 \\
\hline 12575.991 & 110.57 & 7.00 & $\mathrm{~N} / \mathrm{A}$ & 15170.791 & 84.01 & 5.07 & 1.27 \\
\hline 12898.116 & 92.25 & 6.13 & $\mathrm{~N} / \mathrm{A}$ & 15174.093 & 77.01 & 5.12 & 1.26 \\
\hline 13014.818 & 93.28 & 6.34 & $\mathrm{~N} / \mathrm{A}$ & 15187.837 & 72.99 & 5.07 & 1.07 \\
\hline 13015.832 & 84.39 & 6.15 & $\mathrm{~N} / \mathrm{A}$ & 15261.771 & 79.05 & 5.15 & 1.20 \\
\hline 13016.832 & 71.79 & 5.78 & $\mathrm{~N} / \mathrm{A}$ & 15429.120 & 83.25 & 5.07 & 1.17 \\
\hline 13302.975 & 91.91 & 4.79 & 1.34 & 15487.096 & 81.47 & 4.81 & 1.17 \\
\hline 13984.089 & 127.34 & 4.92 & 1.09 & 15522.938 & 88.05 & 4.89 & 0.95 \\
\hline 14130.853 & 128.24 & 5.10 & 1.22 & 15545.819 & 89.25 & 4.89 & 1.35 \\
\hline 14397.938 & 129.88 & 4.87 & 1.04 & 15636.775 & 90.74 & 4.87 & 1.24 \\
\hline 14778.991 & 109.83 & 5.06 & 1.11 & 15879.984 & 113.81 & 4.90 & 1.09 \\
\hline 14790.995 & 103.81 & 5.07 & 1.05 & 15960.761 & 109.29 & 7.51 & 1.28 \\
\hline 14807.917 & 102.51 & 4.97 & 1.02 & 15960.765 & 107.53 & 4.93 & 1.08 \\
\hline 14838.988 & 102.33 & 5.11 & 0.98 & 16019.733 & 113.58 & 4.78 & 1.29 \\
\hline \multicolumn{8}{|l|}{ Hip 57050} \\
\hline 11581.046 & -63.25 & 4.53 & $\mathrm{~N} / \mathrm{A}$ & 15172.138 & -9.58 & 4.64 & 0.81 \\
\hline 11705.827 & -67.09 & 4.79 & $\mathrm{~N} / \mathrm{A}$ & 15174.138 & -14.72 & 4.67 & 0.80 \\
\hline 11983.009 & -9.42 & 5.27 & N/A & 15188.151 & -14.99 & 4.64 & 0.82 \\
\hline 12064.864 & -0.39 & 5.34 & $\mathrm{~N} / \mathrm{A}$ & 15189.155 & 6.60 & 4.25 & 0.86 \\
\hline 12308.077 & 4.98 & 5.01 & $\mathrm{~N} / \mathrm{A}$ & 15190.153 & 25.56 & 4.11 & 0.93 \\
\hline 12391.034 & 6.53 & 5.63 & N/A & 15191.133 & 28.40 & 4.36 & 0.83 \\
\hline 12681.050 & -1.92 & 5.15 & $\mathrm{~N} / \mathrm{A}$ & 15197.136 & 42.23 & 4.28 & 0.79 \\
\hline 12804.885 & 10.26 & 5.05 & $\mathrm{~N} / \mathrm{A}$ & 15198.054 & 35.70 & 4.59 & 0.81 \\
\hline 13077.104 & -38.83 & 5.83 & $\mathrm{~N} / \mathrm{A}$ & 15199.170 & 36.95 & 4.42 & 0.87 \\
\hline 13398.975 & -33.44 & 4.33 & 1.02 & 15229.114 & -28.84 & 4.45 & 0.74 \\
\hline 13753.068 & 12.88 & 4.64 & 0.96 & 15229.958 & -10.72 & 4.71 & 0.72 \\
\hline 14131.092 & 1.32 & 4.96 & 0.90 & 15232.054 & 4.35 & 4.63 & 0.78 \\
\hline 14545.002 & 15.61 & 4.55 & 0.79 & 15251.997 & 0.76 & 4.38 & 0.81 \\
\hline 14546.007 & 22.29 & 4.29 & 0.80 & 15284.858 & 9.29 & 4.64 & 0.73 \\
\hline 14671.811 & -3.54 & 5.13 & 5.32 & 15636.023 & -32.36 & 4.31 & 0.90 \\
\hline 14955.894 & 9.12 & 4.47 & 0.83 & 15671.915 & -13.93 & 4.34 & 0.92 \\
\hline 14963.930 & -13.66 & 4.43 & 0.80 & 15698.820 & 16.56 & 5.97 & 7.95 \\
\hline 15014.782 & -46.65 & 4.50 & 0.70 & 15707.812 & -12.89 & 4.66 & 0.88 \\
\hline 15015.804 & -47.40 & 4.41 & 0.72 & 15723.769 & -34.04 & 4.41 & 0.92 \\
\hline 15041.758 & 11.31 & 5.04 & 0.83 & 15903.064 & 12.42 & 4.40 & 0.83 \\
\hline
\end{tabular}

\section{REFERENCES}

Abell, G. O. 1959, ASPL, 8, 121

Anglada-Escudé, G., Arriagada, P., Vogt, S. S., et al. 2012a, ApJL, 751, L16 Anglada-Escudé, G., Boss, A. P., Weinberger, A. J., et al. 2012b, ApJ, 746, 37 Apps, K., Clubb, K. I., Fischer, D. A., et al. 2010, PASP, 122, 156 Baraffe, I., Chabrier, G., Barman, T. S., Allard, F., \& Hauschildt, P. H. 2003, A\&A, 402, 701

Batalha, N. M., Rowe, J. F., Bryson, S. T., et al. 2013, ApJS, 204, 24 Bean, J. L., Sneden, C., Hauschildt, P. H., Johns-Krull, C. M., \& Benedict, G. F. 2006, ApJ, 652, 1604

Bennett, D. P., Rhie, S. H., Nikolaev, S., et al. 2010, ApJ, 713, 837

Beuzit, J.-L., Ségransan, D., Forveille, T., et al. 2004, A\&A, 425, 997

Bidelman, W. P. 1954, ApJS, 1, 175

Bonfils, X., Delfosse, X., Udry, S., et al. 2013, A\&A, 549, A109

Bonfils, X., Forveille, T., Delfosse, X., et al. 2005, A\&A, 443, L15

Borucki, W. J., Koch, D., Basri, G., et al. 2010, Sci, 327, 977

Borucki, W. J., \& Summers, A. L. 1984, Icar, 58, 121
Bowler, B. P., Johnson, J. A., Marcy, G. W., et al. 2010, ApJ, 709, 396 Bowler, B. P., Liu, M. C., Shkolnik, E. L., et al. 2012, ApJ, 753, 142

Browning, M. K., Basri, G., Marcy, G. W., West, A. A., \& Zhang, J. 2010, AJ, 139,504

Butler, R. P., Johnson, J. A., Marcy, G. W., et al. 2006, PASP, 118, 1685 Butler, R. P., Vogt, S. S., Marcy, G. W., et al. 2004, ApJ, 617, 580

Campo, C. J., Harrington, J., Hardy, R. A., et al. 2011, ApJ, 727, 125

Carson, J., Thalmann, C., Janson, M., et al. 2013, ApJL, 763, L32

Casertano, S., Lattanzi, M. G., Sozzetti, A., et al. 2008, A\&A, 482, 699

Cassan, A., Kubas, D., Beaulieu, J.-P., et al. 2012, Natur, 481, 167

Chabrier, G., Baraffe, I., Allard, F., \& Hauschildt, P. 2000, ApJ, 542, 464

Chang, C.-K., Ko, C.-M., \& Peng, T.-H. 2011, ApJ, 740, 34

Choi, J., McCarthy, C., Marcy, G. W., et al. 2013, ApJ, 764, 131

Crepp, J. R., \& Johnson, J. A. 2011, ApJ, 733, 126

Crepp, J. R., Johnson, J. A., Fischer, D. A., et al. 2012a, ApJ, 751, 97 Crepp, J. R., Johnson, J. A., Howard, A. W., et al. 2012b, ApJ, 761, 39 Crepp, J. R., Johnson, J. A., Howard, A. W., et al. 2013a, ApJ, 774, 1 Crepp, J. R., Johnson, J. A., Howard, A. W., et al. 2013b, ApJ, 771, 46 
Cumming, A., Butler, R. P., Marcy, G. W., et al. 2008, PASP, 120, 531

Currie, T., Debes, J., Rodigas, T. J., et al. 2012, ApJL, 760, L32

Delfosse, X., Forveille, T., Ségransan, D., et al. 2000, A\&A, 364, 217

Dieterich, S. B., Henry, T. J., Golimowski, D. A., Krist, J. E., \& Tanner, A. M. 2012, AJ, 144, 64

Dressing, C. D., \& Charbonneau, D. 2013, ApJ, 767, 95

Dupuy, T. J., Liu, M. C., Bowler, B. P., et al. 2010, ApJ, 721, 1725

Endl, M., Cochran, W. D., Tull, R. G., \& MacQueen, P. J. 2003, AJ, 126, 3099

Faherty, J. K., Burgasser, A. J., Cruz, K. L., et al. 2009, AJ, 137, 1

Femenía, B., Rebolo, R., Pérez-Prieto, J. A., et al. 2011, MNRAS, 413, 1524

Fischer, D. A., Gaidos, E., Howard, A. W., et al. 2012, ApJ, 745, 21

Fischer, D. A., \& Marcy, G. W. 1992, ApJ, 396, 178

Fischer, D. A., \& Valenti, J. 2005, ApJ, 622, 1102

Foreman-Mackey, D., Hogg, D. W., Lang, D., \& Goodman, J. 2013, PASP, 125,306

Gatewood, G. 2008, AJ, 136, 452

Gaudi, B. S., Albrow, M. D., An, J., et al. 2002, ApJ, 566, 463

Gaudi, B. S., Bennett, D. P., Udalski, A., et al. 2008, Sci, 319, 927

Gaudi, B. S., Seager, S., \& Mallen-Ornelas, G. 2005, ApJ, 623, 472

Girardi, L., Bertelli, G., Bressan, A., et al. 2002, A\&A, 391, 195

Gliese, W., \& Jahreiß, H. 1991, in Preliminary Version of the Third Catalogue of Nearby Stars, Technical Report, ed. L. E. Brotzmann \& S. E. Gesser (Greenbelt, MD: NASA/Astronomical Data Center, Goddard Space Flight Center)

Goldman, B., Marsat, S., Henning, T., Clemens, C., \& Greiner, J. 2010, MNRAS, 405, 1140

Golimowski, D. A., Henry, T. J., Krist, J. E., et al. 2004, AJ, 128, 1733

Gomes da Silva, J., Santos, N. C., Bonfils, X., et al. 2012, A\&A, 541, A9

Gould, A., Dong, S., Gaudi, B. S., et al. 2010, ApJ, 720, 1073

Gray, D. F. 1988, Lectures on Spectral-line Analysis: F, G, and K Stars

Gray, R. O., Corbally, C. J., Garrison, R. F., et al. 2006, AJ, 132, 161

Haghighipour, N., Vogt, S. S., Butler, R. P., et al. 2010, ApJ, 715, 271

Henry, T. J., Jao, W.-C., Subasavage, J. P., et al. 2006, AJ, 132, 2360

Henry, T. J., Soderblom, D. R., Donahue, R. A., \& Baliunas, S. L. 1996, AJ, 111,439

Herbig, G. H. 2007, AJ, 133, 2679

Ho, S., \& Turner, E. L. 2011, ApJ, 739, 26

Høg, E., Fabricius, C., Makarov, V. V., et al. 2000, A\&A, 355, L27

Holman, M. J., \& Wiegert, P. A. 1999, AJ, 117, 621

Howard, A. W., Johnson, J. A., Marcy, G. W., et al. 2010a, ApJ, 721, 1467

Howard, A. W., Marcy, G. W., Bryson, S. T., et al. 2012, ApJS, 201, 15

Howard, A. W., Marcy, G. W., Johnson, J. A., et al. 2010b, Sci, 330, 653

Isaacson, H., \& Fischer, D. 2010, ApJ, 725, 875

Jenkins, J. S., Ramsey, L. W., Jones, H. R. A., et al. 2009, ApJ, 704, 975

Johnson, J. A., Aller, K. M., Howard, A. W., \& Crepp, J. R. 2010a, PASP, 122,905

Johnson, J. A., \& Apps, K. 2009, ApJ, 699, 933

Johnson, J. A., Butler, R. P., Marcy, G. W., et al. 2007, ApJ, 670, 833

Johnson, J. A., Gazak, J. Z., Apps, K., et al. 2012, AJ, 143, 111

Johnson, J. A., Howard, A. W., Marcy, G. W., et al. 2010b, PASP, 122, 149

Kaib, N. A., Raymond, S. N., \& Duncan, M. 2013, Natur, 493, 381

Kalas, P., Graham, J. R., Chiang, E., et al. 2008, Sci, 322, 1345

Kass, R. E., \& Raftery, A. E. 1995, J. Am. Stat. Assoc., 90, 773

Kharchenko, N. V. 2001, KFNT, 17, 409

Khrutskaya, E. V., Izmailov, I. S., \& Khovrichev, M. Y. 2010, AstL, 36, 576

Kipping, D. M. 2013, MNRAS, 434, L51

Koch, D. G., Borucki, W. J., Basri, G., et al. 2010, ApJL, 713, L79

Koen, C., Kilkenny, D., van Wyk, F., \& Marang, F. 2010, MNRAS, 403, 1949

Kraus, A. L., \& Ireland, M. J. 2012, ApJ, 745, 5

Kuzuhara, M., Tamura, M., Kudo, T., et al. 2013, ApJ, 774, 11

Lagrange, A.-M., Gratadour, D., Chauvin, G., et al. 2009, A\&A, 493, L21

Landolt, A. U. 1992, AJ, 104, 340

Landolt, A. U. 2009, AJ, 137, 4186

Liu, M. C., Fischer, D. A., Graham, J. R., et al. 2002, ApJ, 571, 519

Lloyd, J. P. 2002, PhD thesis, Univ. California, Berkeley

Luhman, K. L. 2012, ARA\&A, 50, 65
Mann, A. W., Gaidos, E., Kraus, A., \& Hilton, E. J. 2013, ApJ, 770, 43

Mann, A. W., Gaidos, E., Lépine, S., \& Hilton, E. J. 2012, ApJ, 753, 90

Marcy, G. W., \& Butler, R. P. 2000, PASP, 112, 137

Marcy, G. W., Butler, R. P., Fischer, D., et al. 2001, ApJ, 556, 296

Marcy, G. W., Butler, R. P., Vogt, S. S., Fischer, D., \& Lissauer, J. J. 1998, ApJL, $505, \mathrm{~L} 147$

Marois, C., Macintosh, B., Barman, T., et al. 2008, Sci, 322, 1348

Marois, C., Zuckerman, B., Konopacky, Q. M., Macintosh, B., \& Barman, T. 2010, Natur, 468, 1080

Mason, B. D., Wycoff, G. L., Hartkopf, W. I., Douglass, G. G., \& Worley, C. E. 2001, AJ, 122, 3466

Mayor, M., Bonfils, X., Forveille, T., et al. 2009, A\&A, 507, 487

Metchev, S. A., \& Hillenbrand, L. A. 2009, ApJS, 181, 62

Montes, D., López-Santiago, J., Gálvez, M. C., et al. 2001, MNRAS, 328, 45

Mortier, A., Santos, N. C., Sousa, S., et al. 2013, A\&A, 551, A112

Morton, T. D., \& Swift, J. J. 2013, arXiv:1303.3013

Nakajima, T., Oppenheimer, B. R., Kulkarni, S. R., et al. 1995, Natur, 378,463

Napiwotzki, R. 2009, JPhCS, 172, 012004

Neves, V., Bonfils, X., Santos, N. C., et al. 2013, A\&A, 551, A36

Neves, V., Bonfils, X., Santos, N. C., et al. 2012, A\&A, 538, A25

Nielsen, E. L., \& Close, L. M. 2010, ApJ, 717, 878

Oja, T. 1985, A\&AS, 61, 331

Öpik, E. 1924, Publ. Obs. Astron. Univ. Tartu, 25, 6

Pedicelli, S., Bono, G., Lemasle, B., et al. 2009, A\&A, 504, 81

Peña Ramírez, K., Béjar, V. J. S., Zapatero Osorio, M. R., Petr-Gotzens, M. G., \& Martín, E. L. 2012, ApJ, 754, 30

Rameau, J., Chauvin, G., Lagrange, A.-M., et al. 2013, ApJL, 772, L15

Rauscher, E., \& Marcy, G. W. 2006, PASP, 118, 617

Reid, I. N., \& Cruz, K. L. 2002, AJ, 123, 2806

Rivera, E. J., Laughlin, G., Butler, R. P., et al. 2010, ApJ, 719, 890

Rivera, E. J., Lissauer, J. J., Butler, R. P., et al. 2005, ApJ, 634, 625

Robinson, S. E., Laughlin, G., Vogt, S. S., et al. 2007, ApJ, 670, 1391

Rojas-Ayala, B., Covey, K. R., Muirhead, P. S., \& Lloyd, J. P. 2010, ApJL, 720, L113

Rojas-Ayala, B., Covey, K. R., Muirhead, P. S., \& Lloyd, J. P. 2012, ApJ, 748, 93

Rolleston, W. R. J., Smartt, S. J., Dufton, P. L., \& Ryans, R. S. I. 2000, A\&A, 363,537

Scholz, R.-D. 2010, A\&A, 515, A92

Schwarz, G. 1978, AnSta, 6, 461

Siegler, N., Close, L. M., Mamajek, E. E., \& Freed, M. 2003, ApJ, 598, 1265

Skrutskie, M. F., Cutri, R. M., Stiening, R., et al. 2006, AJ, 131, 1163

Southworth, J. 2010, MNRAS, 408, 1689

Stevenson, K. B., Harrington, J., Fortney, J. J., et al. 2012, ApJ, 754, 136

Sumi, T., Bennett, D. P., Bond, I. A., et al. 2010, ApJ, 710, 1641

Sumi, T., Kamiya, K., Bennett, D. P., et al. 2011, Natur, 473, 349

Swift, J. J., Johnson, J. A., Morton, T. D., et al. 2013, ApJ, 764, 105

Tadeu dos Santos, M., Silva, G. G., Ferraz-Mello, S., \& Michtchenko, T. A. 2012, CeMDA, 113, 49

Udry, S., Bonfils, X., Delfosse, X., et al. 2007, A\&A, 469, L43

van Altena, W. F., Lee, J. T., \& Hoffleit, E. D. 1995, in The General Catalogue of Trigonometric Parallaxes, (New Haven, CT: Yale Univ. Observatory)

van Belle, G. T., \& von Braun, K. 2009, ApJ, 694, 1085

van Leeuwen, F. 2007, A\&A, 474, 653

Vigan, A., Patience, J., Marois, C., et al. 2012, A\&A, 544, A9

Vogt, S. S., Allen, S. L., Bigelow, B. C., et al. 1994, Proc. SPIE, 2198, 362

Wheeler, J. C. 2012, ApJ, 758, 123

Wilson, O. C. 1968, ApJ, 153, 221

Winn, J. N. 2011, in Exoplanet Transits and Occultations, ed. S. Piper, 55

Wizinowich, P., Acton, D. S., Shelton, C., et al. 2000, PASP, 112, 315

Wright, J. T. 2005, PASP, 117, 657

Wright, J. T., Marcy, G. W., Fischer, D. A., et al. 2007, ApJ, 657, 533

Wright, J. T., Veras, D., Ford, E. B., et al. 2011, ApJ, 730, 93

Youdin, A. N. 2011, ApJ, 742, 38

Zakhozhaj, V. A. 2002, KFNT, 18, 535 